TOPICAL REVIEW

\title{
Hyperpolarized xenon in NMR and MRI
}

\author{
Ana-Maria Oros and N Jon Shah \\ Institute of Medicine, Research Centre Jülich, 52425 Jülich, Germany \\ E-mail: a.m.oros-peusquens@fz-juelich.de and n.j.shah@fz-juelich.de
}

Received 29 April 2004

Published 24 September 2004

Online at stacks.iop.org/PMB/49/R105

doi:10.1088/0031-9155/49/20/R01

\begin{abstract}
Hyperpolarized gases have found a steadily increasing range of applications in nuclear magnetic resonance (NMR) and NMR imaging (MRI). They can be regarded as a new class of MR contrast agent or as a way of greatly enhancing the temporal resolution of the measurement of processes relevant to areas as diverse as materials science and biomedicine. We concentrate on the properties and applications of hyperpolarized xenon. This review discusses the physics of producing hyperpolarization, the NMR-relevant properties of ${ }^{129} \mathrm{Xe}$, specific MRI methods for hyperpolarized gases, applications of xenon to biology and medicine, polarization transfer to other nuclear species and low-field imaging.
\end{abstract}

\section{Overview}

The growing numbers of applications for laser-polarized ${ }^{129} \mathrm{Xe}$ and ${ }^{3} \mathrm{He}$ in clinical magnetic resonance imaging and in material sciences are predicated on novel research in physics. This has in turn led to the development of more efficient polarizers for the production of large quantities of polarized noble gases. For the brain and biological systems, in particular, hyperpolarized ${ }^{129} \mathrm{Xe}$ is a promising candidate for imaging and spectroscopic applications because Xe dissolves in blood, organic lipophilic solvents and biological tissue and also because Xe shows a large range of chemical shifts. Throughout this review, the reference to the very interesting body of work on hyperpolarized ${ }^{3} \mathrm{He}$ has been minimized only in the interests of space; for an in-depth exposition of hyperpolarized ${ }^{3} \mathrm{He}$ and its applications, readers are directed to authoritative reviews (Kauczor et al 1998, 2001, 2002, Eberle et al 2001, Salerno et al 2001, Leawoods et al 2001, Hedlund and Johnson 2002, Möller et al 2002, Gast et al 2003, Altes and De Lange 2003) and the references therein.

This contribution is organized in the following way. The introduction gives a brief historical overview of the relevant advances which culminated in the development of optical pumping and its application to noble gases. Section 3 reviews the methods for the production of hyperpolarized gases. In section 4, properties of noble gases which are of particular relevance to magnetic resonance imaging (MRI) are discussed. Here, the focus is deliberately placed 
on xenon because, notwithstanding a number of disadvantages, it is of particular interest for in vivo imaging because of its solubility and huge chemical shift. In section 5, a brief overview of NMR in biological systems is presented. The non-renewable nature of the hyperpolarization leads to special requirements when performing MRI experiments; these are discussed in section 6. In section 7, some pertinent MRI results are briefly discussed and references to new results are provided. Polarization transfer and low-field MRI results are discussed in sections 8 and 9, respectively. Finally, in section 10, some perspectives for hyperpolarized gas MRI are given.

\section{Introduction}

The very new and steadily increasing applications of hyperpolarized gases to biology and medicine constitute one of the most impressive examples of physics-life sciences interdisciplinarity. The original development of techniques of laser enhanced nuclear polarization has been motivated by topics in nuclear and particle physics. ${ }^{3} \mathrm{He}$ is one of the simplest nuclei, consisting of only one neutron and a pair of protons which can be considered as a 'spectator'. Since the angular momentum of ${ }^{3} \mathrm{He}$ is determined to a large extent by the neutron, the polarization of a ${ }^{3} \mathrm{He}$ nucleus is given to a large extent by the polarization of its neutron. It is thus the nuclear system closest to a free neutron which can be easily accelerated and polarized.

The question of isospin symmetry (the effect of replacing neutrons by protons and vice versa) plays an important role in nuclear structure. The mass $A=3$ isospin doublet consisting of ${ }^{3} \mathrm{He}$ and the radioactive isotope of hydrogen, ${ }^{3} \mathrm{H}$ (the 'mirror' system of two neutrons and one proton) is the simplest bound system available for the study of isospin symmetry and isospin dependence in nuclear physics.

Polarized beams and targets of ${ }^{3} \mathrm{He}$ therefore have a unique place in the study of spin and isospin dependence of nuclear interactions. Polarized ${ }^{3} \mathrm{He}$ also acts as a spin filter for the polarization of neutrons, by strongly absorbing neutrons with spin antiparallel to its own. The emergent beam is therefore polarized parallel to the ${ }^{3} \mathrm{He}$ polarization. For the production of polarized targets for nuclear reactions, or spin filters, litre quantities of ${ }^{3} \mathrm{He}$ with high $(50 \%)$ polarization are required.

Two methods can be employed for the task of polarizing such large quantities. One is using 'brute force', producing a high Boltzmann polarization based on low temperature, high magnetic field and (in the case of ${ }^{3} \mathrm{He}$, which can only be polarized by this method in its solid state) high pressure. The alternative is to use optical pumping of alkali metal atoms, followed by spin exchange via collisions with the noble gas. In the case of ${ }^{3} \mathrm{He}$, optical pumping can be used directly on a metastable atomic state, followed by resonant exchange of the excitation energy in so-called metastability exchange collisions. A third method involves atomic beams, which can be highly polarized but have densities of $10^{16}$ at best, and is not relevant for the purpose of this review. We address the first two polarization methods in some detail in section 3 , and outline here the historical development which led to the application of optical pumping to noble gases.

Hanle (1924) was the first to show that when the resonance fluorescence of $\mathrm{Hg}$, excited with linearly polarized light, is produced in the presence of an oscillating magnetic field, its degree of polarization decreases and the plane of polarization is rotated (Hanle 1924). A year later, Fermi and Rasetti (1925a) observed depolarization of resonance radiation of $\mathrm{Hg}$ by radiofrequency magnetic fields. This was assigned to the fact that the excited atomic levels were highly polarized by absorption of the polarized light. 
Twenty-five years later, Kastler, aided by Jean Brossel, first his pupil and later close co-worker, was the first to propose a method of investigating Hertzian resonances by optical methods (Brossler and Kastler 1949). These are resonances produced by the interaction of atoms with oscillating radiofrequency waves in a magnetic field, and were discovered by Rabi et al (1938). New profound analysis of the atomic processes connected with the scattering of resonance radiation led Kastler to the method of optical pumping, which he proposed in 1950 (Kastler 1950). In his ground-breaking communication to the Société Française de Physique, he suggested that the population of the sublevels of an atomic ground state can be strongly modified with respect to the equilibrium population by using oriented and polarized light beams. The atoms, excited by the optical radiation, upon returning to the ground state concentrate in certain sublevels and assume preferential orientations in space. The use of this method should allow the orientation of both atoms and atomic nuclei. One of the applications proposed by Kastler in his seminal paper (Kastler 1950) was to use the non-equilibrium orientation of nuclei for the enhancement of the detection of gases or vapours by nuclear magnetic resonance. The experimental confirmation of the unequal population of ground-state sublevels was obtained two years later by Brosseler, Kastler and Winter using an atomic beam of $\mathrm{Na}$ (Brossler et al 1952). These ideas about optical pumping played an important part in the development of the laser. Alfred Kastler received the Nobel Prize in 1966 'for the discovery and development of optical methods for studying Hertzian resonances in atoms'.

The corner stone for using optical pumping for the polarization of noble gases was laid by Happer and co-workers (Happer 1972, Walker and Happer 1997). An enhancement (although modest, below $0.5 \%$ ) of the polarization of a ${ }^{3} \mathrm{He}$ buffer gas in a cell containing optically pumped rubidium was first recognized by Bouchiat et al (1960). The process leading to the polarization of ${ }^{3} \mathrm{He}$ was studied in more detail by Gamblin and Carver (1965) and by Fitzsimmons et al (1969), and was recognized to be predominantly due to spin exchange during very fast binary collisions. The formation of rather long-lived molecules, involving one alkali and one inert gas atom, which is the key process in producing polarization of the heavier noble gases, was for rather a long time either ignored or seen as a relaxation mechanism of the polarized alkali atoms. Grover (1978) detected the polarization enhancement of the heavier noble gases in the presence of polarized $\mathrm{Rb}$ using $\mathrm{Rb}$ as a magnetometer. Continuing this work, Volk et al (1980) recognized the effect of long-lived van der Waals molecules in the spin-exchange process, produced and destroyed in a three body process including $\mathrm{N}_{2}$, but largely overestimate the cross-section for binary processes. The first quantitative determination of the xenon polarization, and of the efficiency of the polarization transfer is due to Bhaskar et al (1982). The spin-transfer efficiency was determined to be $\eta=0.043 \pm 0.006$, which is still quite substantial, although the cross-section for the binary spin-exchange process was found to be reduced compared to the results of Volk, Kwon and Mark by more than an order of magnitude. The possibility of producing large and highly polarized xenon samples by spin exchange with optically pumped alkali metal atoms has thus entered atomic physics, and through the possibility of using highly polarized targets, nuclear physics.

Xenon was first brought into the NMR spotlight by Ito and Fraissard (1980), the pioneers of ${ }^{129} \mathrm{Xe}$ NMR for the study of porous solids. Their aim was to find a molecule which was non-reactive but sensitive to its environment, to physical interactions with other chemical species and to the nature of adsorption sites. Xenon has the largest electron shell among stable noble gases, which is easily deformed. As a consequence, xenon displays the largest range of chemical shifts among all noble gases (Seydoux et al 1993). Furthermore, the isotopes ${ }^{129} \mathrm{Xe}$ and ${ }^{131} \mathrm{Xe}$ are both detectable by NMR, the former with a sensitivity of 0.021 (per nucleus) relative to protons, the latter with a lower sensitivity of $2.7 \times 10^{-3}$. Studies of zeolites 
Table 1. NMR-relevant properties of ${ }^{129}$ and ${ }^{131} \mathrm{Xe}$ : ground-state spin and parity $J_{\text {g.s. }}^{\pi}$, magnetic moment $\mu$, gyromagnetic ratio $\gamma$, quadrupole moment $Q$, natural abundance, sensitivity relative to proton (assuming thermal polarization). Data taken from National Nuclear Data Center.

\begin{tabular}{|c|c|c|c|c|c|c|}
\hline Isotope & $J_{g . s .}^{\pi}$ & $\begin{array}{l}\mu \\
\left(\mu_{\mathrm{B}}\right)\end{array}$ & $\begin{array}{l}\gamma \\
\mathrm{MHz} \mathrm{T}^{-1}\end{array}$ & $\begin{array}{l}Q \\
\text { (b) }\end{array}$ & $\begin{array}{l}\text { Abundance } \\
(\%)\end{array}$ & Sensitivity \\
\hline${ }^{129} \mathrm{Xe}$ & $1 / 2^{+}$ & $-0.7779763(84)$ & -11.7774 & 0.00 & 26.44 & 0.021 \\
\hline${ }^{131} \mathrm{Xe}$ & $3 / 2^{+}$ & $+0.6918619(39)$ & +3.4912 & $-0.120(12)$ & 21.18 & $2.76 \times 10^{-3}$ \\
\hline
\end{tabular}

using thermally polarized Xe have already been highly successful (for a recent review, see Springuel-Huet et al (1999)).

The use of laser polarized ${ }^{129} \mathrm{Xe}$, with its signal increase of four to five orders of magnitude over Boltzmann polarization, greatly facilitates surface studies and the study of dynamics, among others (Pietraß and Gaede 1995, Brunner 1999a). The non-equilibrium magnetization can be sampled with a large number of excitation pulses with very small flip angles in fast succession, before being completely consumed. The need for averaging is practically eliminated, as is the need to wait for $T_{1}$ before repeating the measurement. Both aspects make measurements using thermally polarized xenon very time consuming. In contrast, time resolved spectroscopy and imaging are made possible by hyperpolarized Xe (Pietraß et al 1995, Tseng et al 1999, Mair et al 2000, Moudrakovski et al 2000a, 2000b, 2001b, Appelt et al 2001, Han et al 2004).

Because of the low density of the gas phase, three orders of magnitude below that of water, the magnetization density of hyperpolarized Xe gas is comparable to the equilibrium magnetization of water protons at fields of a few tesla. However, a major advantage offered by hyperpolarized Xe even at gas density is the lack of background, since Xe is not naturally present in the many systems that have been studied. In the solid phase (below $161 \mathrm{~K}$ at $1 \mathrm{~atm})$, the density of Xe ice is comparable to that of water, and the hyperpolarization brings an increase of three to four orders of magnitude in the magnetization density. An alternative solution, which benefits from both increased density over gas phase and the efficiency of Xe as a solvent (Rentzepis and Douglass 1981) is to use liquid Xe for imaging, or as a solvent, for magnetization transfer to other nuclear species. Because of the high magnetization density, combined effects of the distant dipolar field and radiation damping give rise to interesting dynamics in polarized Xe liquid (Jeener 1999, Nacher et al 2000, Sauer et al 2001, Romalis and Ledbetter 2001, Jeener 2002).

The high degree of polarization (50\%) attainable for ${ }^{3} \mathrm{He}$, and its magnetic moment which is substantially larger than that of ${ }^{129} \mathrm{Xe}$ and comparable to that of the proton (see table 1), make gas-phase He imaging quite easy to perform. The method is approaching the stage of being routinely employed in clinical examinations of the lung (Salerno et al 2001).

The all-determining signal-to-noise ratio (SNR) considerations and relaxation behaviour favour use of hyperpolarized gases at low fields. In conventional NMR and MRI, the polarization $P$ of a nuclear species of spin 1/2 and magnetic moment $\mu$ in an applied field B is given by the Boltzmann distribution:

$$
P=\frac{1-\exp \left(-\mu B / k_{\mathrm{B}} T\right)}{1+\exp \left(-\mu B / k_{\mathrm{B}} T\right)} \simeq \frac{\mu B}{2 k_{\mathrm{B}} T}
$$

where $k_{\mathrm{B}}$ is the Boltzmann constant and $T$ is the absolute temperature. At room temperature and a typical clinical field $(1.5 \mathrm{~T})$, the proton magnetization is only $5 \times 10^{-4} \%$. Since the proton has the highest magnetic moment among all known stable isotopes, this is also the highest equilibrium (or thermal) nuclear magnetization attainable with applied magnetic 
fields. However, the magnetization density is $P \times n$ ( $n$ is the proton density) and biological samples consist largely of water. The macroscopic magnetization obtained from, for example, a human head placed in a clinical field, is easily detectable with radiofrequency coils.

The polarization which can be induced in the noble gases via optical pumping is independent of the field strength for subsequent NMR detection. This and NMR-specific noise behaviour (see section 9) lead to the conclusion that the ratio of the SNR obtained from hyperpolarized gases to the SNR obtained from a thermally polarized material (water, for example) is inversely proportional to the field producing the thermal polarization in which detection takes place. With other words, the lower the applied field in which the study takes place, the more beneficial is it to use polarized gases for NMR/MRI instead of protons thermally polarized by that field. A number of studies report one-shot imaging experiments with high SNR, obtained using hyperpolarized ${ }^{3} \mathrm{He}$ or ${ }^{129} \mathrm{Xe}$ at fields approaching Earth's magnetic field. At such a field strength, imaging water protons would require hundreds of thousands of averages and correspondingly long measurement times. Moreover, the transfer of polarization from polarized gases to other elements (see section 8) leads to a higher relative effect at lower applied magnetic fields.

A whole new world of possibilities is thus opened to nuclear magnetic resonance with the use of polarized gases. This review concentrates on the aspects which are relevant to NMR imaging.

A number of excellent reviews are available, highlighting different aspects of the work with hyperpolarized gases. A majority of these reviews concentrate on the applications of polarized xenon since its interaction with many environments make it the more versatile probe. The main field of application of ${ }^{3} \mathrm{He}$, besides polarized targets for nuclear and particle physics, is lung imaging; for relevant reviews see section 1. The production of polarization was reviewed by Happer (1972) and Walker and Happer (1997); several reviews are dedicated to NMR spectroscopy with laser polarized xenon with application to surface studies (Pietraß and Gaede 1995, Pietraß 1999, Brunner 1999a) and biological systems (Brunner 1999b, Goodson 2002, Cherubini and Bifone 2003); one review concentrates on polarization transfer from hyperpolarized xenon (Song 2000); aspects related to the delivery of polarized xenon to biological systems can be found in Goodson (1999); imaging of a variety of systems, with emphasis on living organisms, has been reviewed in Albert and Balamore (1998), Bifone et al (1999), Chupp and Swanson (2001), Mugler et al (2004a) and on low-field imaging in Mair and Walsworth (2002).

\section{Production of hyperpolarization}

The method which has become standard for polarizing noble gases makes use of optical pumping of alkali metals (rubidium in most cases) followed by polarization transfer (spin exchange) to the noble gas nucleus during collisions (Bouchiat et al 1960). A polarization degree of $\approx 10 \%$ is currently standard for Xe polarizers using optical pumping of Rb and spin exchange (Zook and Bowers 2001), with an output close to $1 \mathrm{l} \mathrm{h}^{-1}$. For small quantities, a much higher degree of polarization of $70 \%$ has been reported (Jänsch et al 1998, Ruth et al 1999). A new polarizer (Zook et al 2002) using $210 \mathrm{~W}$ of laser power reports high capacity production of xenon with polarization degree $>65 \%$.

For ${ }^{3} \mathrm{He}$ an additional optical method is available, called metastability exchange pumping (Colegrove et al 1963). It exploits the existence of a metastable atomic level in $\mathrm{He}, 2{ }^{3} \mathrm{~S}_{1}$, which can be optically pumped with an available laser wavelength $(1083 \mathrm{~nm})$. Polarization transfer to the nucleus takes place via the hyperfine interaction. A larger degree of polarization can be attained with this technique than with spin exchange, that is $\sim 50 \%$ for quantities of $0.5-11 \mathrm{~h}^{-1}$ 


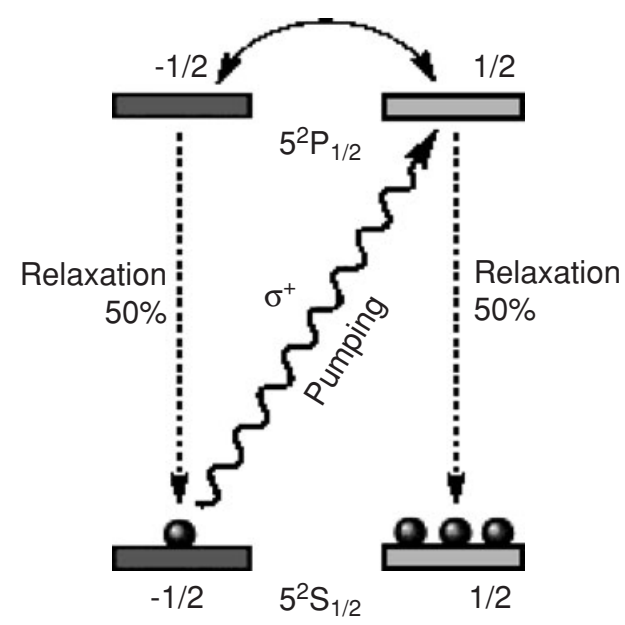

Figure 1. Interaction of alkali atoms with right circularly polarized light leads to non-equilibrium population of the ground-state sublevels. Reproduced with permission from Elsevier from Pietraß (1999).

(Becker et al 1998). This method operates more efficiently at gas pressures around 1 mbar, and quite elaborate compression methods have been devised to ensure a large output (Becker et al 1996). Because of the long relaxation times of ${ }^{3} \mathrm{He}$ gas in specially treated glassware and the large initial polarization, the survival of this high polarization during intermediate-distance transportation ( $24 \mathrm{~h}$ delivery) is perfectly possible. A (European) central production facility of polarized ${ }^{3} \mathrm{He}$ for lungs imaging is being discussed (Van Beek et al 2003).

For both ${ }^{129} \mathrm{Xe}$ and ${ }^{3} \mathrm{He}$, the 'brute force' method seems applicable and holds the promise for the production of large quantities (of the order of 1000 l) of polarized gases per day.

We briefly describe each of the methods below. An in-depth treatment of the subject can be found in the review on spin-exchange optical pumping by Walker and Happer (1997) as well as in the review on optical pumping by Happer (1972). A large part of the review on medical imaging with laser polarized noble gases by Chupp and Swanson (2001) is dedicated to the polarization process. The reader is also referred to the review by Goodson (2002). A brief overview of the existing polarizers and their performances can be found in Zook and Bowers (2001).

\subsection{Spin-exchange optical pumping}

This method takes advantage of the fact that the photons of circularly polarized light have angular momentum with a well-defined projection on the direction of motion (helicity). This projection is $-\hbar$ for left polarized and $+\hbar$ for right polarized light. The interaction of polarized photons with alkali atoms placed in a magnetic field leads to a non-equilibrium population of the magnetic substates of the ground state (optical pumping), as depicted schematically in figure 1 .

At the low fields usually used for polarization, the hyperfine interaction dominates over the Zeeman interaction, and the total angular momentum $\vec{F}=\vec{S}+\vec{I}$ (spin + nuclear angular momenta) as well as its projection are good quantum numbers. Because of the pressure broadening of the absorption line (30 GHz or more (Walker and Happer 1997)) the hyperfine structure of the alkali ground state is not resolved. From a practical point of view, therefore, the situation is similar to that with nuclear spin $I=0$ (note, however, that the two stable isotopes 
of rubidium have nuclear spin $I\left({ }^{85} \mathrm{Rb}\right)=5 / 2$ and $\left.I\left({ }^{85} \mathrm{Rb}\right)=3 / 2\right)$. The two ground-state sublevels with $m_{S}=+1 / 2$ and $m_{S}=-1 / 2$ are shown as degenerate in figure 1 . Interaction with a photon of circularly polarized light leads to a transition to only one of the $m_{J}=+1 / 2$ and $m_{J}=-1 / 2$ substates of the excited state ${ }^{2} \mathrm{P}_{1 / 2}$. Specifically, a photon of right circularly polarized light, $\sigma^{+}$, which carries angular momentum 1 with projection +1 on the direction of the laser and magnetic field, induces a transition between $m_{S}=-1 / 2$ and $m_{J}=+1 / 2$, such that the angular momentum and its projection are conserved. Collisions with noble gas atoms rapidly equalize the populations of the two magnetic substates of ${ }^{2} \mathrm{P}_{1 / 2}$. The depopulating transitions can then proceed with photon emission between states with $\Delta m= \pm 1$, which leads to a mixture of $\sigma^{+}$and $\sigma^{-}$light and the so called radiation trapping, or via non-radiative transitions between states with $\Delta m=0$ induced by collisions with $\mathrm{N}_{2}$ molecules (quenching). For $\mathrm{N}_{2}$ densities of 0.1 amagat or more, radiation trapping is eliminated (Happer 1972). The net result of the process consisting of photon absorption plus collisional mixing plus quenching is to transfer $50 \%$ of the initial population of $m_{S}=+1 / 2$ to $m_{S}=-1 / 2$. Each photon thus adds an average of $1 / 2 \hbar$ per atom. The total alkali-metal atomic spin $\left\langle F_{z}\right\rangle$ grows with time as $\mathrm{d}\left\langle F_{z}\right\rangle / \mathrm{d} t=R_{p}\left(1 / 2-\left\langle S_{z}\right\rangle\right)$ where $R_{p}$ is the absorption rate for unpolarized atoms, which depends on the spectral profiles of the light and the transition line shape.

$\mathrm{Rb}-\mathrm{Xe}$ or $\mathrm{Rb}-{ }^{3} \mathrm{He}$ spin exchange occurs during collisions of a rubidium and a noble gas atom. There are at least two types of collisions (Happer 1972); sudden, binary collisions, which occur on the timescale of $10^{-12} \mathrm{~s}$, and sticking collisions, which lead to molecules bound on a timescale of $10^{-8} \mathrm{~s}$. The binding and destruction process of the van der Waals molecules is a three-body process, involving most often an $\mathrm{N}_{2}$ molecule. While binary processes are dominant in the case of spin exchange with ${ }^{3} \mathrm{He}$, van der Waals molecules contribute mostly to the polarization of the heavier noble gases. Two processes dominate during the lifetime of a bound molecule: spin exchange, via the hyperfine coupling between the spin of the noble gas nucleus and that of the $\mathrm{Rb}$ atom; and spin relaxation, by coupling of the spin of the $\mathrm{Rb}$ atom with the molecular rotation. The cross-sections for these two processes have been calculated for all the possible noble gas-alkali metal pairs, and measured in several cases (with good agreement between theory and measurement) (Walker and Happer 1997).

There are a few processes which produce $\mathrm{Rb}$ spin relaxation, where one of them, spin exchange with the noble gas nucleus, is the desired one. The others are relaxation by $\mathrm{Rb}-\mathrm{Rb}$ collisions, buffer gas molecules other than the noble gas to be polarized, wall interactions and spin-rotation interaction during the alkali-noble gas collision. Typical crosssections for the spin destruction (SD) processes are (Walker and Happer 1997, Allred et al 2002): $\sigma\left(\mathrm{SD} ; \mathrm{Rb}-\mathrm{N}_{2}\right)=10^{-22} \mathrm{~cm}^{2} ; \sigma(\mathrm{SD} ; \mathrm{Rb}-\mathrm{Rb})=9 \times 10^{-18} \mathrm{~cm}^{2} ; \sigma(\mathrm{SD} ; \mathrm{Rb}-\mathrm{He})=$ $9 \times 10^{-24} \mathrm{~cm}^{2} ; \sigma(\mathrm{SD} ; \mathrm{Rb}-\mathrm{Xe})=1.5 \times 10^{-19} \mathrm{~cm}^{2}$. The calculated cross-sections for spin exchange are $\sigma(\mathrm{SE} ; \mathrm{Rb}-\mathrm{Xe})=1.5 \times 10^{-20} \mathrm{~cm}^{2} ; \sigma\left(\mathrm{SE} ; \mathrm{Rb}-{ }^{3} \mathrm{He}\right)=2 \times 10^{-24} \mathrm{~cm}^{2}$. For the polarization of ${ }^{3} \mathrm{He}$, high ${ }^{3} \mathrm{He}$ pressures are therefore needed such that the alkali-He interaction dominates. In the case of xenon, the cross-section for the interaction of $\mathrm{Rb}$ and xenon atoms is large, and so it dominates the spin exchange and relaxation processes.

The polarization of the noble gas nucleus achieved via spin exchange with the alkali metal is given by

$$
\langle P(\text { noble gas })\rangle=\langle P(\text { alkali })\rangle \frac{\gamma_{\mathrm{SE}}}{\gamma_{\mathrm{SE}}+\gamma_{\mathrm{SD}}}
$$

where $\langle P($ alkali $)\rangle$ is the volume-averaged alkali polarization in the pumping cell, $\gamma_{\mathrm{SE}}$ is the spin-exchange cross-section for the alkali-noble gas interaction and $\gamma_{\text {SD }}$ the cross-section for spin destruction of the noble gas (wall interaction, spin-rotation coupling, etc). The maximum value for the polarization of the noble gas is therefore always smaller (or equal, in the ideal case) than that of the alkali metal. 


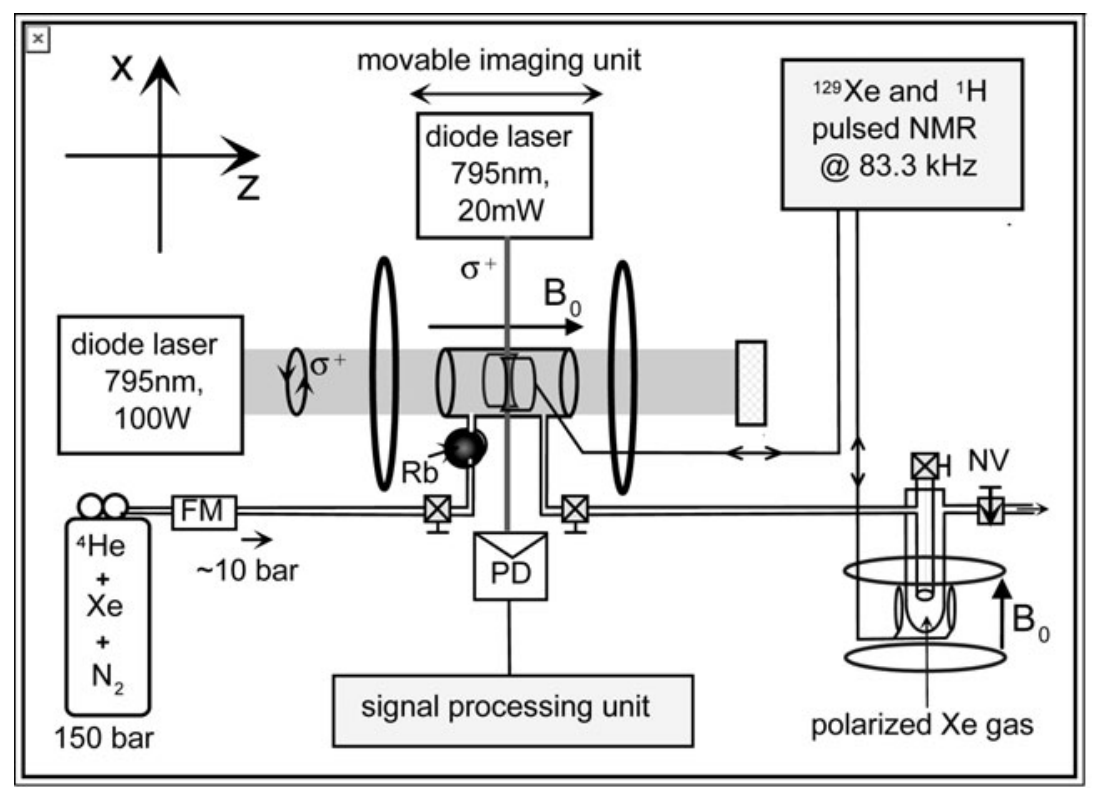

Figure 2. Schematic representation of a $\mathrm{Rb}-\mathrm{Xe}$ polarizer, showing the major components (FM: gas flow meter; PD: photodetector and NV: needle valve). Two diagnostic devices are shown, one measuring the $\mathrm{Rb}$ polarization based on spin temperature (see text), the other the xenon polarization by pulsed NMR. Reproduced with permission from Wiley from Shah et al (2000).

An important feature of the spin-exchange collisions is that they conserve the total angular momentum, but redistribute its projection. Between collisions, the hyperfine interaction redistributes angular momentum between the nucleus and the electron. A common spin temperature, $\beta$ is therefore attained, such that $P=\tanh (\beta / 2)$ (Walker and Happer 1997, Ben-Amar Baranga et al 1998b, Appelt et al 1998). This fact can be used to determine the absolute polarization of $\mathrm{Rb}$ in the pumping cell, by determining the population distribution of the Rb sublevels (Ben-Amar Baranga et al 1998b, Saam and Conradi 1998, Shah et al 2000).

Schematically, the components of a polarizer are: (a) a glass cell with specially prepared surface; (b) gas mixture consisting of the vapour of the alkali metal ( $\mathrm{Rb}$ usually), the noble gas to be polarized and buffer gas consisting of nitrogen or nitrogen and ${ }^{4} \mathrm{He}$; and (c) the laser system. The whole system is placed in a magnetic field, parallel to the direction of laser light, with strength ranging from a few tens of Gauss produced by Helmholtz coils (e.g. Saam and Conradi 1998), to a few hundred Gauss in the fringe fields of NMR magnets (e.g. Rosen et al 1999, Fukutomi et al 2003) or inside solenoid coils (Bhaskar et al 1982), to normal NMR fields of a few T (Augustine and Zilm 1996, 1997). A typical polarizer is shown in figure 2.

Each component has to be carefully optimized, resulting in a rather complicated, multiparametric problem. To illustrate the results which are attainable with different set-ups, we show in figure 3 the polarization flow as a function of laser power for the polarizers summarized by Zook and Bowers (2001). The polarization flow is defined as $P[\%] \cdot(\mathrm{Xe}$ flow $[\mathrm{sccm}])$, and is the quantity $\left(\right.$ in $\mathrm{cm}^{3}$ ) of polarized ${ }^{129}$ Xe per minute times 100 . We address the optimization process below.

The wavelength of the $\mathrm{Rb}$ resonance line $D 1$, used for optical pumping, is $794.7 \mathrm{~nm}$, which translates to a frequency of $377.24 \mathrm{THz}$. Pressure broadening contributes around $18 \mathrm{GHz} /$ amagat to the linewidth (Chupp and Swanson 2001). Ideally, the laser frequency 


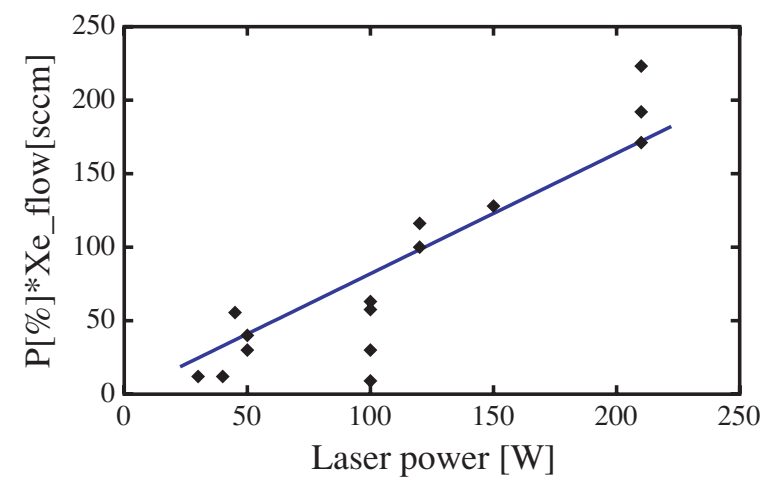

Figure 3. Output of polarized ${ }^{129} \mathrm{Xe}$ as a function of laser power. The data on the performances of different polarizers are taken from Zook and Bowers (2001).

and linewidth would be identical to that of the $\mathrm{Rb}$ line. The powers needed are of the order of tens of $\mathrm{W}$, depending on the absorption linewidth of $\mathrm{Rb}$ vapour in the polarizer and the linewidth of the laser. This amount of power can be provided by (a few) Ti::sapphire lasers or by diode laser arrays (LDAs). The two solutions are dramatically different in cost ( $1 \mathrm{~W}$ of laser power from LDAs is a factor of 100 time cheaper than from Ti::Sa), such that LDAs vastly dominate in the construction of polarizers. The bandwidth of an LDA is typically 2-4 nm, much larger than the $\sim 0.1 \mathrm{~nm}$ pressure-broadened $\mathrm{Rb}$ line, making the light absorption rather inefficient. However, the convolution of the absorption cross-section with the emission profile ensures that light $1 \mathrm{~nm}$ off-resonance still effectively contributes to the Rb polarization.

The laser power is chosen such that the polarization losses of the $\mathrm{Rb}$ atoms are compensated by optical pumping. The dimensions of the polarizing vessel should ensure that for the used laser power a nearly homogenous illumination is attained, resulting in a nearly uniform $\mathrm{Rb}$ polarization.

Special coating of the glass is needed since wall surface relaxation is an important factor of polarization loss. Wall relaxation leads, for example, to significant differences between the $\mathrm{Rb}$ and the Xe degree of polarization (Shah et al 2000). Surface relaxation on normal glass is dominated by the interaction with paramagnetic impurities (usually iron content), the sticking time (adsorption energy), as well as diffusion in the case of ${ }^{3} \mathrm{He}$. The use of Corning or Pyrex glasses with low impurity concentration is standard. Smith et al (1998) report relaxation times for ${ }^{3} \mathrm{He}$ reaching bulk values ( $250 \mathrm{~h}$ at 3 amagat) for single cells made of Corning 7056 glass. Various coatings are used for helium and xenon polarizer and storage vessels. Cesium coating of diffusion-tight Supremax (aluminosilicate) glass was shown to increase the relaxation time of ${ }^{3} \mathrm{He}$ to up to $120 \mathrm{~h}$ (Surkau et al 1997). The high xenon density polarizer built by Rosen et al (1999) uses octadecyltrichlorosilane (OTS) as a coating; it is shown that for magnetic fields above $20 \mathrm{G}$ and at the temperatures used in the polarizer, the wall relaxation rate is much smaller than the rate of spin exchange. The same coating is used for the storage and delivery vessels and results in long relaxation times of the xenon ice $(60 \mathrm{~min})$ and xenon gas (18 min). Silicone (Surfrasil) wall coatings have long been proved to be efficient for xenon (Zeng et al 1985, Driehuys et al 1995, Sauer et al 1999). The latter two publications of the Princeton group (Driehuys et al 1995, Sauer et al 1999) investigate the interaction of xenon with the protons contained in the coating by double-resonance mechanisms and polarization transfer. They demonstrate that Xe wall relaxation in the coated cell is: (a) very small $\left(T_{1}\right.$ of $10^{3} \mathrm{~s}^{-1}$ at $20-100 \mathrm{G}$ ); (b) strongly field dependent for fields below $20 \mathrm{G}$; and (c) is due to dipolar relaxation to the protons contained by Sufrasil. 
The alkali vapour and gas mixture are kept at constant temperature by an oven. The temperature also has to be optimized (Appelt et al 1999), since most quantities which influence the degree of polarization depend on it. A value of $120 \mathrm{C}$ is found to ensure a high $\mathrm{Rb}$ polarization as well as its uniform distribution over the cell (Shah et al 2000), for a typical xenon polarizer (Driehuys et al 1996, Shah et al 2000). Rb is usually chosen since its high vapour pressure allows operation at relatively low temperature, avoiding chemical corrosion of the glass container and reducing diffusion-mediated wall relaxation.

Among the alkali metals, an increased use of $\mathrm{K}$ instead of $\mathrm{Rb}$ can be expected in the future (Chupp and Swanson 2001). This is due to the fact that the spin-exchange rate, which results in polarization of the noble gas, is similar for $\mathrm{K}$ and $\mathrm{Rb}$, whereas the loss of alkali metal polarization due to spin-rotation interaction is nearly an order of magnitude higher for $\mathrm{Rb}$ than for K (Kadlecek et al 1998, Ben-Amar Baranga et al 1998a). In fact, a dual K-Rb polarizer for ${ }^{3} \mathrm{He}$ has been recently reported by Babcock et al (2003), in which $\mathrm{Rb}$ is optically pumped, followed by spin transfer to $\mathrm{K}$, which transfers polarization to ${ }^{3} \mathrm{He}$. For a K-rich vapour, a polarization efficiency of $1 / 4$ is achieved, which is an order of magnitude higher than for $\mathrm{Rb}$ alone.

The composition of the buffer gas also plays an important role. The cross-section for depolarizing the $\mathrm{Rb}$ atoms by collisions is smallest for (natural composition) He, followed by $\mathrm{H}_{2}, \mathrm{Ne}$ and $\mathrm{N}_{2}$, but with cross-sections larger by one or more orders of magnitude. The additional gases contribute to different optimization steps; whereas $\mathrm{N}_{2}$ is mainly used to quench the $\mathrm{Rb}$ fluorescence, $\mathrm{He}$ is used to pressure-broaden the absorption line of $\mathrm{Rb}$ atoms, and thus make more efficient use of broad-band diode lasers power, as well as for decreasing the effect of wall collisions. Both the collisional broadening and the suppression of wall collisions increase with pressure. On the negative side, the depolarization of $\mathrm{Rb}$ or $\mathrm{Xe}$ atoms by collisions with the buffer gas increases as well. In practice, ${ }^{4} \mathrm{He}$ pressures of about $10 \mathrm{~atm}$ are employed (Driehuys et al 1996, Shah et al 2000), limited only by the integrity of the glass cell. The gas composition is optimized in this case to $98 \% \mathrm{He}$ and $1 \%$ each of $\mathrm{N}_{2}$ and Xe (Driehuys et al 1996). A high pressure polarizer has been patented (Shah et al 2001a) and is currently being built in Jülich. The polarizer uses a two-chamber system comprising of an outer pressure vessel, which holds the main polarizer components, and the pumping cell. Through the simultaneous regulation of pressure within the pumping cell and in the pressure vessel, it is straightforward to attain high pressure (30-50 atm) in the cell and yet maintain a negligible pressure gradient across the cell wall. The high density Xe polarizer of Rosen et al (1999) uses 1700 Torr highly purified natural xenon, with 150 Torr $\mathrm{N}_{2}$ and no additional buffer gas. They report $157 \mathrm{~cm}^{3}$ xenon polarized to $7.5 \%$ within $5 \mathrm{~min}$ ( 1 batch). Recently, Fukutomi et al (2003) reported a polarizer working at atmospheric pressure. A polarization degree of $35 \%$ is reported for ${ }^{129} \mathrm{Xe}$, achieved with $20 \mathrm{~W}$ of laser power and an optimized gas mixture of $5 \% \mathrm{Xe}, 10 \% \mathrm{~N}_{2}$ and $85 \% \mathrm{He}$ at atmospheric pressure. The high degree of polarization is contrasted with the few per cent only, which were obtained when no buffer gases were added.

One of the sources of polarization loss within the polarizer is radiation trapping. Absorption of polarized photons by the alkali atoms (or metastable He) can be followed by reemission of photons of the same energy (resonant scattering). Light radiated by these atoms is nearly unpolarized. When the mean free path for the photons is much less than the dimensions of the polarizer, the unpolarized light can be reabsorbed by further Rb atoms, effectively resulting in a polarization loss. This effect gives rise to a laser-power-dependent term in the relaxation rate. The easiest solution is to add $\mathrm{N}_{2}$ gas, which is chemically inert, at high pressure. Collisions between excited $\mathrm{Rb}$ atoms and buffer gas molecules produce non-radiative quenching of the excited states, thus avoiding emission of resonant unpolarized light. 
The magnetic field dependence of the polarization efficiency was investigated by Augustine and Zilm (1997, 1996), who suggested that the use of high fields will increase the polarization. The use of a high magnetic field reduces radiation trapping, since the Zeeman splitting of the $\mathrm{S}$ and $\mathrm{P}$ states causes the photons with the 'wrong' polarization to be off-resonance and produce very little depolarization. Furthermore, the spin-exchange cross-section is practically field independent, whereas the $T_{1}$ of Xe increases with the field. In a recent study, Jau et al (2002) measured the ${ }^{129} \mathrm{Xe}-\mathrm{Rb}$ spin-exchange rate coefficient at $9.4 \mathrm{~T}$ and $160^{\circ} \mathrm{C}<T<200^{\circ} \mathrm{C}$, using the Faraday rotation method to measure accurately the $\mathrm{Rb}$ density. The rate was determined to be $\kappa=\frac{\mathrm{d}\left(1 / T_{1}\right)}{\mathrm{d}[\mathrm{Rb}]}=(1.75 \pm 0.12) \times 10^{-16} \mathrm{~cm}^{3} \mathrm{~s}^{-1}$, showing practically no temperature dependence, and about $20 \%$ lower than the (calculated) rate at zero field and $100{ }^{\circ} \mathrm{C}$. Although the rate is smaller than those reported by Cates et al (1992) at $1.1 \times 10^{-5} \mathrm{~T}$ and Augustine and Zilm (1997) at $2.35 \mathrm{~T}$, the conclusion about the more efficient polarization in high field still holds. In addition, Walter et al (2002) showed recently that the spin relaxation due to binary collisions of alkali-metal atoms with buffer gas atoms is also slowed down by increasing magnetic fields. Polarizing the sample in the same strong field in which it is measured has the further advantage of avoiding the sample manipulation and transport through magnetic field gradients, which can cause substantial loss of polarization. However, due to the cost effectiveness of using low fields, this is the solution adopted in the majority of polarizers based on optical pumping.

Magnetic field gradients have to be minimized, since diffusion in varying magnetic fields leads to rapid relaxation. At high pressure, the field gradient contribution to the relaxation rate of the spin polarization is given by

$$
\Gamma_{\nabla B}=\frac{\left|\nabla B_{\perp}\right|^{2} \cdot D}{B_{0}^{2}}
$$

where $\nabla B_{\perp}$ is the magnetic field gradient in the transversal plane, $D$ is the diffusion coefficient, and $B_{0}$ is the constant field in which the polarization process takes place. This relaxation rate is inversely proportional to the pressure, through the dependence of the diffusion coefficient.

The polarizers can operate in either batch or continuous flow mode. In order to obtain the rather large quantities needed for medical applications, for example, several batches have to be accumulated. The polarized xenon is stored as ice in a cold finger kept at liquid nitrogen temperature. An important source of relaxation in xenon ice at low fields is cross relaxation to ${ }^{131} \mathrm{Xe}$ (Gatzke et al 1993). The effect can be minimized by increasing the storage field, or by annealing (Lang et al 2002). Much longer relaxation times, and thus larger quantities of accumulated xenon, could be attained by keeping the storage finger at liquid He temperature.

\subsection{Metastability exchange}

Excited He atoms can be produced by an rf discharge in a glass bulb containing He gas at low pressure. The metastable $2{ }^{3} \mathrm{~S}_{1}$ state, with around $10^{-6}$ of the population in the steady state, can be optically pumped, as demonstrated for ${ }^{4} \mathrm{He}$ gas by Colegrove and Franken (1960). The circularly polarized pumping light, which induces transitions from ${ }^{3} \mathrm{~S}_{1}$ to the level ${ }^{3} \mathrm{P}_{0}$, creates non-equilibrium populations of the ${ }^{3} \mathrm{~S}_{1}$ sublevels. The available laser wavelength of $1083 \mathrm{~nm}$ is required for pumping. Resonant exchange of the excitation energy in metastability exchange collisions occurs on a timescale much shorter than that of hyperfine interaction and thus does not affect the nuclear spin. The ground-state population therefore attains large polarizations (Colegrove et al 1963). Under optimum conditions, a polarization degree of $80 \%$ can be attained for ${ }^{3} \mathrm{He}$ samples at low densities $\left(10^{16} \mathrm{~cm}^{-3}\right)$ (Chupp and Swanson 2001). 


\subsection{Cryogenic method}

Frossati (1998a) proposed a method for obtaining quantities of the order of 100-1000 1 polarized gas per day. ${ }^{3} \mathrm{He}$ solidifies only at temperatures below $320 \mathrm{mK}$ in conjunction with pressures of 29 bar or higher. Liquid He is not significantly polarized by magnetic fields in the available range. However, in an applied field of $15 \mathrm{~T}$ and temperatures of $4 \mathrm{mK}$, solid ${ }^{3} \mathrm{He}$ reaches a polarization degree of $95 \%$. The highly polarized solid ${ }^{3} \mathrm{He}$ is produced by compressing liquid $\mathrm{He}$ in an external magnetic field until it starts solidifying below $320 \mathrm{mK}$. Since the entropy of the liquid is lower than that of the solid, solidification produces cooling (Pomeranchuck cooling). If the solid is melted in a time much shorter than $T_{1}$ (which is several hundreds to several thousand seconds at the relevant temperatures), the magnetization necessarily remains in the liquid. Frossati's suggestion for obtaining polarized gas from the liquid is to extract the Pomeranchuck cell in 1 to $2 \mathrm{~s}$ from the centre of the magnet, to a region where the temperature is 6 to $7 \mathrm{~K}$ and where the liquid evaporates through a teflon coated or glass filling line, to a storage vessel. The main source of relaxation is then crossing the high gradient field at the edges of the magnet and this can be minimized by making the time rather long (around $1 \mathrm{~s}$ ). Since the size of the cell is limited only by the size of the magnet, this method could produce around $1000 \mathrm{l}$ of polarized ${ }^{3} \mathrm{He}$ per day, which would not only be sufficient for medical applications, but could be used to fuel a $1 \mathrm{GW}$ fusion power plant. For deuterium (and possibly for xenon), a different method was proposed by Frossati. Ortho-deuterium, with a small admixture of para-deuterium, was condensed in ${ }^{3} \mathrm{He}$, and was observed to polarize to $13 \%$ within $5 \mathrm{~min}$. For a higher degree of polarization, the grains of molecular ice grains must be of the order of micron.

The equilibrium polarization of ${ }^{129} \mathrm{Xe}$ in a field of $16 \mathrm{~T}$ and temperatures of $15 \mathrm{mK}$ is $29 \%$. The main challenge is to polarize large quantities of xenon in short amounts of time. The long relaxation times at $\mathrm{mK}$ temperature, of several days, are a problem (time consuming) during the polarization step, but become a benefit for the storage of polarization. Biškup et al (2003) recently proposed a way of polarizing xenon films using a refined version of the 'brute force' method. It uses dilution refrigerator temperatures and a high magnetic field of $8 \mathrm{~T}$. The very long $T_{1}$ values of $\mathrm{Xe}$ at $\mathrm{mK}$ temperatures are reduced by the relaxation agent ${ }^{3} \mathrm{He}$. The nuclear spins of a surface immersed in liquid ${ }^{3} \mathrm{He}$ are rapidly relaxed by it, via the quantum tunnelling of ${ }^{3} \mathrm{He}$ atoms in the solid-like layer formed near solid surfaces. The relaxation time for surface ${ }^{129} \mathrm{Xe}$ spins therefore depends on the spectral density of tunnelling in the localized ${ }^{3} \mathrm{He}$ layer at the frequency of ${ }^{129} \mathrm{Xe}$ at $8 \mathrm{~T}$ and on the distance between the xenon and helium nuclei. A large surface is created by plating the xenon onto a substrate with very high surface area, as silica gel. At temperatures below $0.1 \mathrm{~K}$, the relaxation time of xenon on the silica gel surface is larger than $2 \times 10^{4} \mathrm{~s}$, and increases strongly with decreasing temperatures $\left(T_{1} \propto T^{-1.7}\right)$. When the surface is immersed in liquid ${ }^{3} \mathrm{He}$, the relaxation times are only around $1000 \mathrm{~s}$ and show no temperature dependence. After the equilibrium polarization is attained, a method of switching off the relaxation process was suggested by addition of ${ }^{4} \mathrm{He}$ to the cell (known to preferentially occupy sites near the surfaces). A number of obstacles, however, still need to be addressed before this method can compete with the optical one for producing large quantities of xenon gas.

\subsection{Polarimeters}

In order to obtain reproducible results, it is necessary to control the degree of polarization attained and preserved in as many of the different steps of an experiment as possible. Within 
the polarizer, it is important to characterize either the steady-state polarization of the gas, or the polarization in the storage finger (for xenon ice).

The first measurement of the polarization of xenon induced by the spin-exchange with $\mathrm{Rb}$ vapour was performed by Bhaskar et al (1982). The same paper recognizes the potential of the method and characterizes the processes leading to xenon polarization. The polarimetry is performed by adiabatically inverting the Xe magnetization; the NMR signal was compared with that from a bottle of distilled water contained in an identical vessel and measured at the same positions in the NMR coils and at the same frequency (smaller applied field). This method is very widely used by the diagnostic devices of the polarizers with either adiabatic passage or pulsed NMR.

In order to estimate the efficiency of the polarization process, the $\mathrm{Rb}$ as well as the $\mathrm{He}$ or Xe polarization should be measured. This is done by determining the spin temperature of the $\mathrm{Rb}$ atoms. The population of the ground-state sublevels is measured by detecting the RFmodulated, laser-light absorption (Ben-Amar Baranga et al 1998a, Saam and Conradi 1998, Shah et al 2000).

An indirect method for measuring the polarization of liquid xenon has been proposed recently by Verhulst et al (2002). It uses the dipolar field created by liquid hyperpolarized ${ }^{129} \mathrm{Xe}$ which is homogeneous in the bulk of the liquid and adds to the external field. The corresponding shift in the Larmor frequency of the proton in $\mathrm{CHCl}_{3}$ (chloroform), which dissolves homogeneously in xenon over a fairly broad temperature range, is a very good measure of xenon polarization. ${ }^{13} \mathrm{C}$ can also be used as a probe, but the frequency shift is four times lower than that of protons, reflecting the lower $\gamma$ value. Direct measurement involves measuring a signal from Xe at the beginning of the experiment and at the end, when thermal equilibrium is reached, and is quite imprecise. The method based on the dipolar-field induced chemical shift is faster than the direct measurement, since the $T_{1}$ of protons $(\sim 20 \mathrm{~s})$ is much shorter than that of liquid xenon $(>15 \mathrm{~min}$ ), and quite sensitive. Liquid xenon with $\sim 1 \%$ polarization in the set-up of Verhulst et al (2002) induces a frequency shift of $6 \mathrm{~Hz}$ for the chloroform protons, allowing the determination of the polarization degree with a precision of $15 \%$. Furthermore, the ${ }^{129} \mathrm{Xe}$ polarization is minimally disturbed, since only the proton response is measured and chloroform is a fairly weak source of ${ }^{129} \mathrm{Xe}$ relaxation. The method can also be used for xenon in concentrated solutions.

Two high precision methods for measuring the polarization of ${ }^{3} \mathrm{He}$ were reported by Romalis et al (1998) the usual direct method using adiabatic fast passage, and an indirect method measuring the shift of the Rb Zeeman resonance. The direct method was calibrated using the Boltzmann polarization of a water probe. After carefully correcting for the effects of water relaxation during the field sweep (which affects both the height and the shape of the water signal and makes the signal dependent on the speed and direction of the magnetic field sweep), the differences in shape of the He and water sample, and different positions in the coil, an accuracy of $3.4 \%$ was reported. The indirect method consists of measurement of the shift in the $\mathrm{Rb}$ Zeeman resonance (electron paramagnetic resonance, EPR). The size of the shift is large, $20 \mathrm{kHz}$, compared to the Zeeman frequency of $9.3 \mathrm{MHz}$ in a field of $20 \mathrm{G}$. The shift in the EPR frequency is due to both the magnetic field generated by He polarization, as well as the $\mathrm{Rb}-\mathrm{He}$ spin exchange interaction. Both magnetic fields which produce the shifts are proportional to the He polarization and density, and can be combined in a single proportionality constant for a given cell shape: $B_{\mathrm{He}}=8 \pi / 3 \kappa_{0} \mu_{\mathrm{He}}\left[{ }^{3} \mathrm{He}\right] P$. In order to determine the absolute polarization, $\kappa_{0}$ was measured and the sign of the polarization was changed periodically, by adiabatic fast passage. The total error of this method was reported by Romalis et al to be $3 \%$. The density of ${ }^{3} \mathrm{He}$ could also be determined with high precision $(\sim 2 \%)$ and independently from the same methods. 


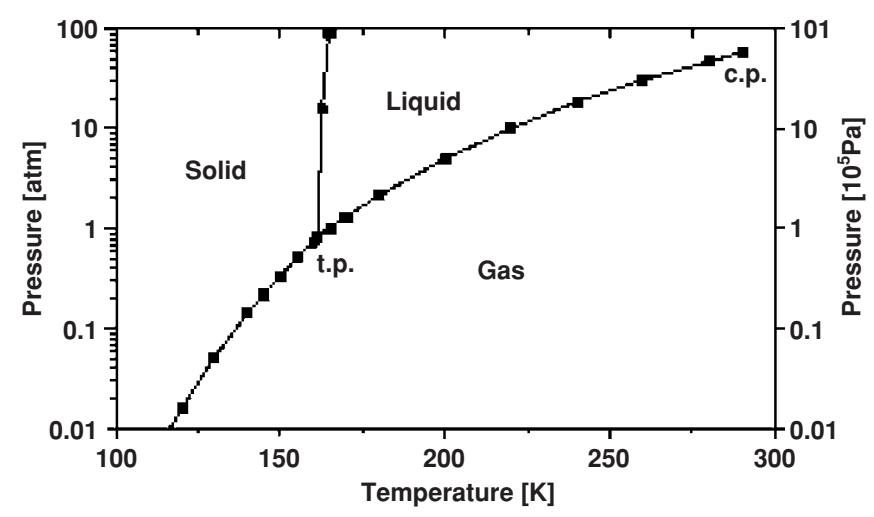

Figure 4. Phase diagram of xenon. Reproduced with permission from Cherubini and Bifone (2003).

\section{MR-relevant properties of hyperpolarized gases}

\subsection{General properties}

Xenon ('xenos' is Greek for 'stranger') was discovered by Sir William Ramsay and Morris Travers in 1898 in the residue left after evaporating liquid air components. Xenon is present as a trace gas in the atmosphere, at concentrations of $0.087 \mathrm{ppm}$. This production method is still the one used commercially.

As seen from figure 4, the three phases of Xe are all accessible in rather benign laboratory conditions. It is gaseous at standard temperature and pressure but can can be readily liquefied (boiling point: $165.1 \mathrm{~K}$ at $1 \mathrm{~atm}$ ) and frozen (melting point $161 \mathrm{~K}$ at $1 \mathrm{~atm}$ ). Under moderate pressures of a few tens atm, Xe is a liquid at temperatures below $17^{\circ} \mathrm{C}$ (critical point).

Most applications of hyperpolarized xenon use its gaseous form, but experiments with liquid (Sauer et al 1997, Fitzgerald et al 1998, Tseng et al 1999), frozen (Raftery et al 1992, Gatzke et al 1993, Bowers et al 1993, Song et al 1998a, 1998b, Augustine et al 1998) and supercritical xenon (Haake et al 1998, Leawoods et al 2000) have been reported. Hyperpolarization allows for a much enhanced time resolution. Several studies of the phase dynamics of xenon are also available (Tseng et al 1999, Mair et al 2000, Appelt et al 2001, Han et al 2004).

Xe turns out to be an efficient solvent in its liquid and supercritical phase with densities at least three orders of magnitude higher than the gas phase (Rentzepis and Douglass 1981, Haake et al 1998, Leawoods et al 2000). Furthermore, the long relaxation times for the polarization ( $T_{1}$ of 20 min (Sauer 1997) in liquid, and hundreds of seconds in the supercritical state (Haake et al 1998)), make liquid or supercritical xenon the ideal medium for studying polarization transfer to other NMR-active elements (Leawoods et al 2000, Cherubini et al 2003).

Xenon has an atomic number of $Z=54$ and an atomic mass of 131, resulting from a combination of nine naturally occurring isotopes, with masses ranging from 124 to 136 . Seven of them are stable and two very long lived radioactive ones $\left({ }^{124} \mathrm{Xe}\right.$, natural abundance $0.1 \%, T_{1 / 2} \geqslant 1.6 \times 10^{14}$ years and ${ }^{136} \mathrm{Xe}$, natural abundance $8.9 \%, T_{1 / 2} \geqslant 3.6 \times 10^{20}$ years). The radioactive isotope ${ }^{133} \mathrm{Xe}$ (ground-state spin-parity $3 / 2^{+}, T_{1 / 2}=5.24$ days) is frequently used in nuclear medicine. The remainder of this work concentrates on ${ }^{129} \mathrm{Xe}$ and ${ }^{131} \mathrm{Xe}$ which are both stable and NMR active with natural abundances of $26.4 \%$ and $21.29 \%$, respectively. 
Table 2. Ostwald coefficient of xenon, $L$, in different substances of biological relevance. Data taken from Clever (1979).

\begin{tabular}{|c|c|c|c|c|}
\hline$T(\mathrm{~K})$ & Substance & Preparation & $L$ & $\frac{\text { Mol Xe }}{\text { Mol solvent }}$ \\
\hline 310.15 & Water & - & $0.0834(2)$ & \\
\hline \multirow[t]{3}{*}{310.15} & Plasma & Air & $0.091(2)$ & \\
\hline & & $\mathrm{N}_{2}$ & $0.090(2)$ & \\
\hline & & $\mathrm{O}_{2}$ & $0.093(2)$ & \\
\hline \multirow[t]{3}{*}{310.15} & Erythrocytes & air & $0.190(8)$ & \\
\hline & & $\mathrm{N}_{2}$ & $0.200(8)$ & \\
\hline & & $\mathrm{O}_{2}$ & $0.170(10)$ & \\
\hline 298.15 & Albumin & - & 0.2382 & \\
\hline 303.15 & & - & 0.1954 & \\
\hline 310.15 & & - & 0.1493 & \\
\hline 298.15 & Fat & - & 2.0606 & \\
\hline 303.15 & & - & 1.9630 & \\
\hline 310.15 & & - & 1.8345 & \\
\hline \multirow[t]{3}{*}{294.15} & Albumin & - & - & 1.1 \\
\hline & Haemoglobin & - & - & 1.9 \\
\hline & Methaemoglobin & - & - & 1.9 \\
\hline \multicolumn{5}{|c|}{ Other solvents } \\
\hline \multirow[t]{2}{*}{310.15} & $0.9 \% \mathrm{NaCl}$ & - & $0.078(7)$ & \\
\hline & Olive oil & - & $1.79(4)$ & \\
\hline
\end{tabular}

The basic NMR properties of the two xenon isotopes are given in table 1. The isotope ${ }^{129} \mathrm{Xe}$ has a gyromagnetic ratio which is about four times higher than that of ${ }^{131} \mathrm{Xe}$, leading to a much higher sensitivity. The lack of spectroscopic quadrupole moment for the spin 1/2 isotope ensures that the relaxation times are generally much longer than for ${ }^{131} \mathrm{Xe}$. It is therefore not surprising that of the two xenon isotopes, the one used in the vast majority of applications is ${ }^{129} \mathrm{Xe}$

Although noble gases are known as 'inert gases', evidence has been gathered during the past 40 years for the existence of noble gases compounds. Xe forms several: difluoride, hexafluoride, sodium perxenate, tetrafluoride, xenon deuterate and xenon hydrate. For the vast majority of applications, however, Xe can be considered chemically inert.

\subsection{Solubility}

The solubility of Xe, defined in terms of the Ostwald coefficient (the ratio of volume of gas at its partial pressure in the liquid to the volume of absorbing liquid), is particularly high for organic solvents (4.8 for hexane, 3.1 for benzene, 3.3 for fluorobenzene (Clever 1979)). Most notably, Xe is highly soluble in lipids, with an Ostwald coefficient in oil or fat tissue more than one order of magnitude higher that that for Xe in water (see table 2).

The mole fraction solubility $x_{1}$ is defined as the ratio $x_{1}=n_{g} /\left(n_{g}+n_{l}\right)$ with $n_{g}, n_{l}$ the number of moles of gas and liquid, respectively, at the same temperature and gas partial pressure of $1 \mathrm{~atm}$. The temperature dependence of the mole fraction solubility $x_{1}$ of Xe in water, obtained from an empirical best fit to experimental data in the temperature range 273-353 K, is given by Clever (1979):

$$
\ln \left(x_{1}\right)=-74.7398+105.210 /(T / 100 \mathrm{~K})+27.4664 \ln (T / 100 \mathrm{~K}) .
$$

The solubility shows a broad minimum with predicted minimum temperature of $380 \mathrm{~K}$. 
We show in table 2 values illustrating the solubility of Xe in substances which are relevant for biological systems. Most values were determined using the radioactive isotope ${ }^{133} \mathrm{Xe}$ and are taken from the evaluation by Clever (1979). Note the dependence of xenon solubility not only on the temperature but also on the oxygenation state of blood components.

The reduced solubility of $\mathrm{Xe}$ in water is due to the fact that noble gases belong to hydrophobic species, being electrically neutral and nonpolar, and thus preferring neutral and nonpolar solvents or molecular environments as lipids.

Liquid water is considered to be a macroscopically connected, random network of hydrogen bonds, which is constantly reorganizing (Stillinger 1980). Insertion of a hydrophobic species into water reduces the number of configurations available for the water molecules in its vicinity. These will rearrange themselves around the non-polar molecule or atom in a convex hydrogen bond polyhedron of a large enough size to accommodate the solute molecule. These cages or cavities are an initial stage of clathrate, but the water molecules composing them still experience substantial motion.

The low solubility of nonpolar molecules in water, compared to that in organic liquids, can be understood on the basis of molecular dynamics simulations of the distribution of cavities in water and hexane. The excess chemical potential, $\mu^{\mathrm{exc}}$, of a solute molecule, defined as the quasi-static work needed to bring this molecule from the gas phase into the solvent, becomes larger for water than for organic solvents precisely at solvent sizes of the order of the smallest atomic diameter $(1.3 \AA$ ) (Pohorille 1998). This is due to the fact that the fractional free volume of water is distributed in smaller cavities than the free volume in hexane.

\subsection{Anaesthesia}

One of the most interesting properties of xenon is its action as a general anaesthetic. This observation emerged in 1939, from a study of the 'drunkeness' effect in deep-sea divers, performed by Behnke for the US Navy (Marx et al 2000). The first use of xenon as an anaesthetic was reported in 1946 on mice (Lawrence et al 1946). Xenon produces anaesthesia in humans at MAC values (defined as minimal alveolar concentrations to produce an anaesthetic effect in $50 \%$ of the patients) of $71 \%$ atm (Cullen et al 1969). It has several advantages over other anaesthetic agents; it is non-teratogen (does not generate physical defects in offspring in utero), does not contribute to the depletion of the ozone layer, has analgesic effects at subanaesthetic concentrations ( $\sim 20 \%$ ), offers good circulatory stability, better regional perfusion of individual organs than other agents, does not influence body temperature, and has rapid washout (Marx et al 2000). Its effect on the cerebral blood flow (some anaesthetic agents increase $\mathrm{CBF}$ and disrupt cerebral autoregulation) is still being debated. Apart from its completely reversible anaesthetic action, xenon is thus benign to the organism; the only obstacle to widespread clinical use is its high cost (approx. $\$ 101^{-1}$ ) (Marx et al 2000).

Although it is generally agreed that ion channels are the ultimate site of action of general anaesthetics, there is still considerable disagreement about the molecular mechanisms. A common view is that anaesthetics disrupt the actions of ion channels indirectly, by modifying physical properties of the lipid bilayer. The alternative view is that anaesthetics act by directly binding to specific sites on ion channel proteins. The target sites are therefore either the lipid portion of neural membranes, or the hydrophobic pockets in certain crucial excitable proteins (Franks and Lieb 1994). Irrespective of the exact nature of the interactions, it is often assumed that the site of action is hydrophobic in nature. This is due to the observation that the potency of general anaesthetics correlates with their solubility in lipids, such as olive oil.

The first report of anaesthetic potency being related to lipid solubility was published by Meyer (1899), followed two years later by a similar theory published independently by Overton 
(1901). Meyer and Overton had discovered the most striking correlation observed between the physical properties of general anaesthetic molecules and their potency. The potency of many agents, defined as the reciprocal of the molar concentration required to induce anaesthesia in tadpoles, was compared with their olive oil/water partition coefficient. Meyer and Overton found a nearly linear relationship between potency (which is independent of the means by which the anaesthetic was delivered - either gas or aqueous phase) and the partition coefficient for many types of anaesthetic molecules; alcohols, among others. Apart from this striking correlation, general anaesthetics comprise an exceedingly diverse range of compounds having no obvious chemical or structural similarities (Franks and Lieb 1987).

There is a limitation to the Meyer-Overton correlation, the so-called cutoff effect. Alcohols with increasingly longer carbon chain become increasingly more hydrophobic, and indeed increasingly more potent anaesthetics, as expected from the Meyer-Overton law. However, at certain chain lengths, the addition of just one methylene group causes the molecule to lose its ability to anaesthetize. The cutoff effect is easily interpreted if target sites for general anaesthetics are hydrophobic pockets of fixed dimensions in proteins.

Early attempts were made by Miller et al (1981) to investigate the site of anesthetic action based on the chemical shift of xenon in a range of biological systems-lipid bilayers, biomembranes and myoglobin. They concluded that, on the basis of their results, it is not possible to rule out lipid as the site of anaesthetic action, as suggested Franks and Lieb (1978). However, they reinforced the idea that, given xenon's unusually high sensitivity to its environment, an in-depth study of the chemical shifts and relaxation properties of xenon in bilayers and proteins is likely the best contribution of NMR to the study of anesthesia.

Mitchell Albert (Albert and Balamore 1998) confesses, in a historical perspective on the introduction of hyperpolarized gases to medicine-related application, that similar considerations lead him to dedicate himself to work involving xenon NMR. Hyperpolarized xenon is arguably the single best-suited agent to clarifying anaesthesia.

\subsection{Availability}

The supply of xenon from the atmosphere (concentration $0.087 \mathrm{ppm}$ ) provides the necessary amounts for the moment. Being dense and easily ionized and having a high propulsive capacity, xenon is the ideal gas for positioning satellites, and gradually replaces chemical propellants for satellite-positioning ion motors. This demand will probably influence the price of xenon (currently around $\$ 101^{-1}$ at natural composition; the much higher price of $\$ 3001^{-1}$ for $75 \%$ enrichment in ${ }^{129} \mathrm{Xe}$ is due to limited demand).

\subsection{Chemical shift}

If a bare nucleus in vacuum is subjected to an applied field, its Larmor frequency will be different from that of a nucleus surrounded by its electrons and the rest of the medium. This is called chemical shift, $\sigma$. The precession of electrons from the electron cloud in an applied magnetic field is equivalent to an electric current, producing a field which opposes the applied field. Furthermore, the external field polarizes the electronic shells, producing an additional magnetic field (and electric, see below) at the nucleus. Further contributions result from interactions of the electron shell with those of the surrounding atoms (medium dependent). The chemical shift is in general a tensor, but only its time averaged component along the external field is observable.

The large polarizability of xenon relative to that of other rare gases means that Xe can be expected to have significant interactions with its environment, but not to be chemically or 


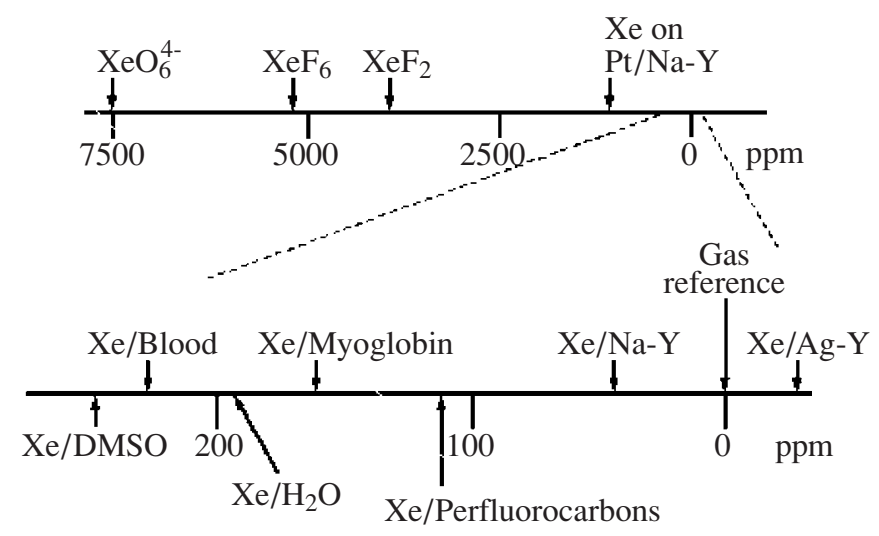

Figure 5. Chemical shifts of xenon in solution and compounds. Reproduced with permission from Elsevier from Goodson (2002) (adapted from Pietraß and Gaede (1995)).

structurally disruptive (Rentzepis and Douglass 1981). As illustrated by figure 5, xenon has a chemical shift range of roughly $350 \mathrm{ppm}$ in solution and more than $7000 \mathrm{ppm}$ in compounds, making it an ideal probe of its chemical and physical environment.

A recently observed effect (Meersmann and Haake 1998) further illustrates the high polarizability of the Xe electron cloud. Using gaseous as well as liquid ${ }^{131} \mathrm{Xe}$ (liquid just below the critical point of $289 \mathrm{~K}$ and $5.8 \mathrm{MPa}$, where $T_{1}$ is $110 \mathrm{~ms}$ ) and a high magnetic field, Meersmann and Haake observed (a) the existence of a quadrupolar splitting for the spin $3 / 2{ }^{131} \mathrm{Xe}$, of the order of a few $\mathrm{Hz}$ for magnetic fields above $10 \mathrm{~T}$; and (b) an increase in the quadrupolar splitting with magnetic field. The bulk of the effect cannot be attributed to the previously observed effect of the surface interaction (Wu et al 1987), as demonstrated by its presence with nearly unchanged magnitude in the gas as well as in the liquid phase with vastly different volume/surface ratios. It has therefore been concluded that the electric field gradient at the nucleus was produced by the same mechanism of magnetic field-electron shell interaction which generates the usual chemical shift. The magnitude of the effect is rather surprising for a noble gas atom with closed electron shell.

Polarizability plays a further important role in Xe binding to protein, and transport through protein interior. While the atomic diameter of Xe is $4.42 \AA$, McKim and Hinton found evidence for xenon transport through a $4 \AA$ diameter gramicidin channel (McKim and Hinton 1994). Very interesting is also the conclusion reached by Xu and Tang (1997) following their study of the site of anesthetic action using the nuclear overhauser effect (NOE) to highlight the interaction between xenon and specific protons on a model biomembrane. Thus, they conclude, the selection of molecular target sites depends not only on the properties of the anaesthetic molecule itself, but also on possible changes in these properties after the interaction with a membrane takes place. The xenon atom is apolar and would be expected to be more compatible with the core of the lipid bilayer. However, the interface was determined to be the site of the interaction. This is explained to be caused by the induced dipole that makes xenon more adaptable to an amphiphilic region of the model biomembrane under study.

The chemical shift is an environment-characteristic NMR property much easier to measure than relaxation times, or their distribution. The sensitivity of the chemical shift of Xe to its environment has found a broad range of applications.

The chemical shift of Xe is usually referenced to xenon gas extrapolated to zero density. $\mathrm{Xe}$ in its liquid phase has a chemical shift of about $240 \mathrm{ppm}$ and in the solid phase of about 
$300 \mathrm{ppm}$. The influence of pressure and temperature on the chemical shift in gas, liquid and solid, as well as the underlying mechanisms have been studied.

Streever and Carr (1961) and then Hunt and Carr (1963) studied the range of chemical shifts and longitudinal relaxation rates in gaseous and liquid xenon. Their observations showed that the chemical shift is accurately proportional to density over a range extending from rarefied gases well into the liquid phase, and determined the proportionality constant to be $0.422 \mathrm{ppm}$ (the density being expressed in amagat). The expression for the chemical shift and the relaxation times agrees well with a relationship derived by Torrey (1963). The explanation lies in the spin-rotation coupling, that is, the rotation of the distorted electron clouds during the collision of two xenon atoms. The electron clouds become distorted from the van der Waals interaction which takes place during collisions, giving rise to an additional chemical shift. This additional magnetic field is also time dependent, due to the rotation of the distorted electron clouds during collisions; relaxation arises from the coupling of the nuclear spin with the time-dependent field. This spin-rotation coupling plays a major role among the processes invoked in explaining the relaxation and chemical shift of xenon.

The study of the chemical shift in xenon gas was extended by Jameson, Jameson and collaborators (Jameson et al 1970, 1973). In the gaseous phase, the chemical shift is due to collisions between Xe atoms, which have a deshielding effect, and is temperature and pressure dependent. With increasing pressure, the rate of $\mathrm{Xe}-\mathrm{Xe}$ interactions and thus the deshielding effect of collisions increases, and so does the chemical shift. The chemical shift dependence on the temperature and pressure of the Xe gas, as determined by Jameson et al, has the general expression:

$$
\sigma=\sigma_{0}(T)+\sigma_{1}(T) \rho+\sigma_{2}(T) \rho^{2}+\cdots,
$$

with $\sigma_{0}$ being the reference shift (temperature independent), $\sigma_{1}=-0.553 \mathrm{ppm} /$ amagat at $298 \mathrm{~K}$ and showing temperature dependence (decreasing as the temperature increases), and $\sigma_{2}$ of the order of $10^{-4} \mathrm{ppm} /$ amagat $^{2}$ can be neglected for pressures below $\sim 100$ amagat. At very low pressures the chemical shift is also sensitive to the interaction with the walls, lending itself to the study of microporous materials.

Figure 6 illustrates the behaviour of the chemical shift of gaseous Xe with density and pressure in the vicinity of the critical point. The data were obtained by Haake et al (1998), who investigated the use of supercritical laser-polarized xenon for NMR studies. The experimental values agree very well with theoretical predictions (Jameson et al 1973).

Xe NMR plays a major role in the study of microporous materials, as exemplified for zeolites in a recent review by Springuel-Huet et al (1999). For the chemical shift of xenon in zeolites at room temperature, a detailed empirical formula has been deduced by Fraissard and Ito (1988), taking into account the different influences to which Xe is subjected while interacting with the microporous material. The different terms have different dependence on Xe concentration, different signs, and different behaviour with the number of adsorption sites, potentially providing detailed information on the zeolite structure. Julbe et al (1999) have evaluated the potential of ${ }^{129} \mathrm{Xe}$ NMR for characterizing the structure of amorphous microporous materials, and determined the influence of porosity on the shape and slope of the variation of the xenon chemical shift. Dissolved-phase dynamics and time-dependent diffusion can be used to obtain information about surface-to-volume ratio and tortuosity in porous media (Mair et al 1999, Butler et al 2002, Mair and Walsworth 2002).

Sensitivity of Xe to the macroscopic structure of its surroundings has been demonstrated by Raftery et al (1992) for thin films of polarized Xe ice. The films, frozen on the walls of glass cells of different geometries, showed line shapes which are dependent on the geometry of the cell, as well as on the orientation of the cell with respect to the applied magnetic field. 

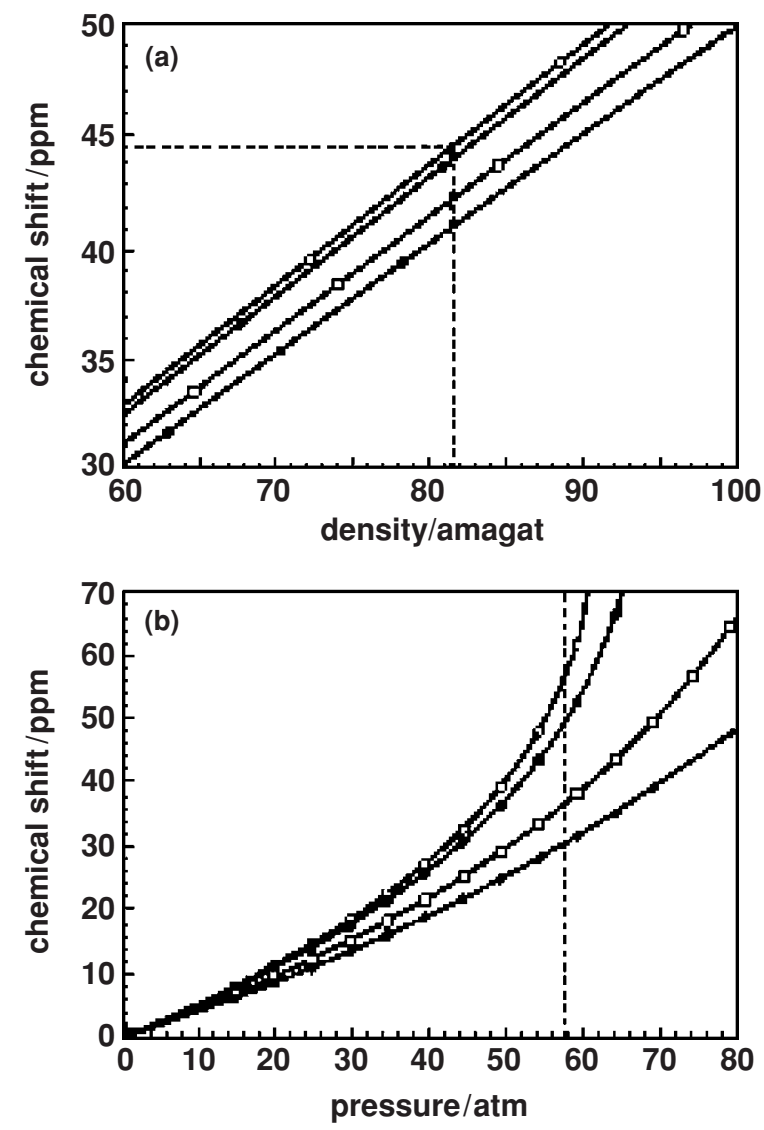

Figure 6. Measured chemical shift of gaseous xenon as a function of density (a) and pressure (b) at $22{ }^{\circ} \mathrm{C}$ (circles), $27^{\circ} \mathrm{C}$ (bullets), $47^{\circ} \mathrm{C}$ (squares) and $67{ }^{\circ} \mathrm{C}$ (diamonds). The curves were calculated from the temperature and density dependence of the ${ }^{129} \mathrm{Xe}$ chemical shift described by Jameson et al (1973). The dashed lines in (a) indicate the experimental conditions of Haake et al (1998) after disappearance of the supercritical phase due to cooling (slightly subcritical at $54 \mathrm{~atm}$ ), while the dashed line in (b) shows the critical pressure of xenon (57.6 atm). Reproduced with permission from Elsevier from Haake et al (1998).

Xenon plays an interesting role in the study of water structures due to its hydrophobic properties mentioned above. In this case, it is not only the probe of the environment, but inherent to the formation of the ordered, cage-like structures of water molecules. Studies involving both thermally polarized and hyperpolarized xenon, which allowed resolution of the dynamics of clathrate formation, have been performed (Ripmeester and Ratcliffe 1990, Pietraß et al 1995).

The self-diffusion of water is also influenced by the formation of such ordered structures, and a retardation factor of 1.3 for the slow component was measured by Haselmeier et al (1995). Measurements of diffusion of Xe in water using thermally polarized Xe are particularly difficult and error-prone due to the long relaxation times and the low sensitivity, which requires signal averaging. Using hyperpolarized Xe, and a BURST sequence, Wolber et al (1998) determined the Xe diffusion coefficient in water $D=2.2(4) \times 10^{-5} \mathrm{~cm}^{2} \mathrm{~s}^{-1}$ with good accuracy, and in good agreement with the coefficient measured by Haselmeier et al for the 'retarded' water. 
The high sensitivity of the chemical shift of xenon to subtle changes in its environment allows the use of functionalized Xe as a biosensor, as developed by Spence et al (2001). The most stable xenon-host complex known to date involves a cage-like molecule, cryptophane-A. Xenon enclosed within the cage displays an up-field chemical shift of $71 \mathrm{ppm}$. Depending on the target which needs to be detected via changes in the xenon chemical shift, a ligand head which shows high affinity to the target is chosen. The cage and the ligand head are connected by a tether. Spence et al exemplify this method for the interaction of the biotin as ligand head with avidin as a target. A shift of $1 \mathrm{ppm}$ up-field compared to xenon bound to the bare cage is observed for 'functionalized' xenon (cage + tether + ligand head), and a shift of $2.3 \mathrm{ppm}$ down-field signalizes the binding of biotin to avidin. A variety of such complexes could be engineered for specific applications.

\subsection{Exchange between sites}

The large range of Xe chemical shifts also facilitates the study of exchange between sites. Kinetic information about the exchange is contained in the transverse relaxation times, and thus in the resulting line shapes. By monitoring the line shapes and their dependence on different parameters (temperature, concentration and field strength), information about properties characterizing each environment and the exchange rates between them can be obtained (Pietraß and Gaede 1995, Springuel-Huet et al 1999, Tilton and Kunz et al 1982).

In a two-site model, $\mathrm{A}$ and $\mathrm{B}$, each site is characterized by its chemical shift $\nu_{\mathrm{A}}$ and $\nu_{\mathrm{B}}$, population $P_{\mathrm{A}}$ and $P_{\mathrm{B}}$, intrinsic transverse relaxation time $T_{2 \mathrm{~A}}$ and $T_{2 \mathrm{~B}}$ and residence time $\tau_{\mathrm{A}}$ and $\tau_{\mathrm{B}}$, respectively. Depending on the exchange rates and chemical shifts, a process can be in slow, intermediate, or fast exchange. In the limit of slow exchange, which is met when $(\tau \Delta \omega)^{2} \gg 1$, a separate line is obtained for each site. Here $\tau$ is the average residence time of the state and equals the inverse of the exchange rate $\tau=k_{\mathrm{ex}}^{-1}$, and $\Delta \omega=2 \pi \Delta \nu$ is the chemical shift difference between the states. The linewidth of each resonance is given by

$$
\pi \Delta v_{i}=\frac{1}{T_{2 i}}+\frac{1}{\tau_{i}} .
$$

As the exchange rate increases (or if $\Delta \omega$ decreases, for example with decreasing magnetic field), the linewidth increases, reaching a maximum where the two lines merge into a single broad one. The condition for intermediate exchange is $(\tau \Delta \omega)^{2} \sim 1$.

The single line narrows as the exchange rate increases further, until the fast exchange condition is met: $(\tau \Delta \omega)^{2} \ll 1$. The linewidth for the fast exchange regime is given by

$$
\pi \Delta v=\frac{1}{T_{2}}=\frac{P_{\mathrm{A}}}{T_{2 \mathrm{~A}}}+\frac{P_{\mathrm{B}}}{T_{2 \mathrm{~B}}}+P_{\mathrm{A}}^{2} P_{\mathrm{B}}^{2}(\Delta \omega)^{2}\left(\tau_{\mathrm{A}}+\tau_{\mathrm{B}}\right) .
$$

Tilton and Kunz applied the above formalism to describe the binding of $\mathrm{Xe}$ to protein (myoglobin and haemoglobin) (Tilton and Kunz 1982). A lower limit of $3 \times 10^{5} \mathrm{~s}^{-1}$ could be determined for the exchange rate of Xe with methaemoglobin (one binding site), and an upper limit for the exchange time of $\tau=k_{\text {off }}^{-1}=3.3 \mu \mathrm{s}$. The interaction of Xe with myoglobin was found to exhibit a multiple binding site pattern; using the likely assumption that one of the binding sites is similar to that in haemoglobin, an exchange rate of $9.6 \times 10^{4}$ was estimated for the additional site.

\subsection{Relaxation times}

A long longitudinal relaxation time for the isotope ${ }^{129} \mathrm{Xe}$ in gaseous form has already been observed in 1950, by Proctor and Yu (1950), who measured its magnetic moment by NMR 
techniques. They used paramagnetic powders as a catalyst of nuclear relaxation. The enhanced signal of hyperpolarized xenon has greatly simplified the measurement of relaxation times in recent years by eliminating the need for substantial averaging and long repetition times required in experiments with thermal polarization.

Early data on the $T_{1}$ values of xenon gas were provided by Brun et al (1954a, 1954b), who noted that the relaxation time of ${ }^{129} \mathrm{Xe}$ was much shorter than expected from dipolar interactions alone, whereas calculations agreed well with the measured relaxation time of ${ }^{131} \mathrm{Xe}$ (dominated by the quadrupole interaction).

Detailed information about the relaxation behaviour of xenon was gained from the studies of (Streever and Carr 1961), followed by Brinkmann et al (1962) and Hunt and Carr (1963). Streever and Carr determine the density dependence of the relaxation time in gas as $T_{1} \sim \rho^{-2.1 \pm 0.4}$ for pressures between 48 and $73 \mathrm{~atm}$, with the maximum measured $T_{1}$ of $2600 \pm 600 \mathrm{~s}$ at $48 \mathrm{~atm}$. The authors discuss possible relaxation mechanisms and on the basis of the measured density dependence conclude that the dominant contribution, as for the chemical shift, is provided by the fluctuating magnetic field sampled by the spin during atomic collisions.

A recent study by Moudrakovski et al (2001a, 2001b) investigated the longitudinal relaxation of thermally as well as laser-polarized ${ }^{129} \mathrm{Xe}$ gas as a function of temperature, field and density. The main relaxation mechanism at high densities was confirmed to be spinrotation, as identified by the older studies (Streever and Carr 1961, Hunt and Carr 1963). For lower pressures, which can only be efficiently investigated using hyperpolarized xenon, they suggested that wall effects become dominant below 3 amagat. It was furthermore confirmed that scalar interaction with ${ }^{131} \mathrm{Xe}$ does not contribute significantly to ${ }^{129} \mathrm{Xe}$ relaxivity in the gas phase. A new contribution to the relaxivity of xenon was identified from the field-dependent effects in the modulation of the chemical shift tensor.

A subsequent study by Chann et al (2002) identified the spin-rotation coupling within bound $\mathrm{Xe}-\mathrm{Xe}$ van der Waals molecules as the primary relaxation process at densities below 14 amagat. Using laser-polarized xenon and measuring relaxation times as a function of gas composition in mixtures of xenon with $\mathrm{He}, \mathrm{Ar}$ and $\mathrm{N}_{2}$, a clear increase in relaxation time was noted with increasing buffer gas density ratio. The authors were able to separate $\mathrm{Xe}-\mathrm{Xe}$ relaxation from wall relaxation. The pure $\mathrm{Xe}-\mathrm{Xe}$ molecular relaxation rate corresponds to $T_{1}=4.1 \mathrm{~h}$; for fixed gas composition, the relaxation rate is independent of gas density (for low densities). For higher densities, the binary relaxation mechanism becomes important, as previously established. When both molecular and binary contributions are considered, the gas relaxation rate in pure xenon is determined to be $\Gamma=6.7 \times 10^{-5} \mathrm{~s}^{-1}+5.0 \times 10^{-6} \mathrm{~s}^{-1}$ [Xe] (with Xe density in amagat), showing that molecular relaxation exceeds binary relaxation below 14 amagat. Previous studies at fixed isotopic composition were not able to identify this molecular mechanism, and wall relaxation was consequently overestimated. Interestingly, the molecular relaxation can be reduced by high fields; the field at which the relaxation is reduced by a factor of 2 was estimated to be $44 \mathrm{~T} /$ amagat. Chann et al also estimate the properties of the wall which produces a $T_{1}$ of around 1 day, which would allow comfortable storage/transport of polarized xenon. For a mixture Xe: $\mathrm{N}_{2}$ of $1: 10$ and a 101 spherical glass chamber, a relaxivity of $2 \times 10^{-5} \mathrm{~cm} \mathrm{~s}^{-1}$ or less is required for the glass wall, a value that is already within the range of known materials.

Relaxation in liquid xenon was first studied by Hunt and Carr (1963), who obtain values of around $16 \mathrm{~min}$, practically independent of field and temperature in the range -72 to $10^{\circ} \mathrm{C}$. The relaxation rate depends linearly on density, with a proportionality factor of approximately $5 \times 10^{-6}$.

$$
1 / T_{1}=(5.0 \pm 0.5) \times 10^{-6} \rho(\mathrm{Xe})
$$


Hunt and Carr conclude that neither dipolar coupling between ${ }^{129} \mathrm{Xe}$ atoms, nor the electron-mediated scalar interaction with ${ }^{131} \mathrm{Xe}$ can give the right order of magnitude of the observed relaxation rates. Using a simple random walk model, they estimate the relaxation rate arising from spin-rotation coupling and obtain

$$
1 / T_{1}=36 \cdot\left(\gamma \frac{m c}{e}\right)^{2} \cdot D \cdot\langle\Delta \sigma\rangle_{t}^{2} / R_{\mathrm{m}}^{2}
$$

where $m$ and $e$ are the electronic mass and charge, respectively, $c$ is the speed of light, $\gamma$ is the gyromagnetic factor of xenon, $D$ is the diffusion coefficient, $\langle\Delta \sigma\rangle_{t}$ is the observed chemical shift, time averaged over the duration of the collision, and $R_{\mathrm{m}}$ is the radius of the hard sphere used to model collision. All three variable quantities, $T_{1},\langle\Delta \sigma\rangle_{t}$ and $D$ are NMRmeasurable quantities. Since the product $D\langle\Delta \sigma\rangle_{t}^{2}$ is measured to be approximately constant with temperature for xenon liquid, this explains the constancy of the measured relaxation rates. The chemical shift (in ppm) is also field independent, explaining the independence of the relaxation rate. The more recent results of Diehl and Jokisaari (1990a) confirmed the field independence of $T_{1}$. The spin-rotation coupling is therefore the main mechanism responsible for relaxation in pure liquid xenon, as well as in gas.

As a caveat it is noted that the liquid samples have to be very pure; the relaxation rate was found to be very sensitive to the concentration of paramagnetic oxygen impurity (Hunt and Carr 1963), with the dependence given by (compare the coefficient to that of equation (8))

$$
1 / T_{1}=(0.56 \pm 0.15) \cdot \rho\left(O_{2}\right) \text {. }
$$

Relaxation studies of solid xenon were performed by Yen and Norberg (1963). The transverse relaxation time in $\mathrm{Xe}$ ice as well as liquid was investigated, and its dependence on temperature. The authors measure a $T_{2}$ value of $1 \mathrm{~ms}$ for xenon ice at $4.2 \mathrm{~K}$, which represents the pure dipolar rigid lattice relaxation time. Above temperatures of $117 \mathrm{~K}$, xenon displays motional narrowing, and the $T_{2}$ values increase by three orders of magnitude (exponentially in $(-1 / T(\mathrm{~K})))$ before transition to the liquid state occurs. In the liquid state, the transverse relaxation time varies little with temperature and takes values close to $10 \mathrm{~s}$. More recently, Tseng et al (1999) measured relaxation in polarized liquid Xe at a field of $4.7 \mathrm{~T}$ and temperature of $166 \mathrm{~K}$. They observed values of roughly $25 \mathrm{~min}$ for the longitudinal relaxation time and larger than $1 \mathrm{~s}$ for the transverse relaxation time. The values were thought to be typical for ${ }^{129} \mathrm{Xe}$ throughout its liquid phase, as previously established by Yen and Norberg (1963).

However, in addition to solid or liquid helium at low temperatures, hyperpolarized liquid xenon represents an ideal system for studying the rather odd behaviour of systems with high magnetization density (one effect being the appearance of multiple echoes following only two RF pulses and in the presence of a gradient field (Deville et al 1979)). Because of the very large nuclear magnetization of liquid xenon, and as a manifestation of the combined effect of long range dipolar interaction and radiation damping (Lin et al 2000), the NMR signal following strong radiofrequency pulses presents an unexpected chaotic behaviour, resulting in an abrupt decay of the transverse magnetization (Jeener 1999, 2002, Nacher et al 2000, Sauer et al 2001). Suppression of these instabilities led Romalis and Ledbetter (2001) to a particularly stable, highly magnetized system. They determined a value of $T_{2}=1300 \mathrm{~s}$ for liquid ${ }^{129} \mathrm{Xe}$, roughly equal to $T_{1}$ and much longer than the values determined by Yen and Norberg (1963) or Tseng et al (1999). This is also the longest transverse relaxation time measured in a liquid.

While the spin-rotation coupling was found to be the main relaxation mechanism in pure liquid xenon, for xenon in solutions the situation can be different. Luhmer et al (1995) calculated, by molecular dynamics simulation, the dipolar relaxation time of Xe in benzene. The contribution of the dipolar mechanism was found to be $30-60 \%$ of the total relaxation. 
The environment can thus be probed through the effect of dipolar coupling on relaxation (sensitive to concentrations), for example using xenon dissolved in different solutions.

The transverse relaxation times of xenon in solution are quite long. Wolber et al (1998) determine $T_{2}$ values of $8.5 \mathrm{~s}$ for polarized xenon in benzene and $15 \mathrm{~s}$ for xenon in PFOB. Zhao et al (1998) measure $T_{2}$ of $5 \mathrm{~s}$ for xenon in water.

While most studies concentrate on the isotope ${ }^{129} \mathrm{Xe}$, this is not exclusively the case. A precise determination of the magnetic moments of ${ }^{133} \mathrm{Xe}$ in its ground and ${ }^{131,133} \mathrm{Xe}$ in their isomeric $11 / 2^{-}$state (Calaprice et al 1985 ) was made possible by NMR detection of the very large signal due to spin exchange with optically pumped $\mathrm{Rb}$ atoms in a polarizer when steady state was achieved. Because of the dominating quadrupolar interaction, these spin $3 / 2$ isotopes $\left({ }^{131,133} \mathrm{Xe}\right)$ have short $T_{1}$ relaxation times, not allowing for a substantial degree of polarization to build up with a batch method. However, the quadrupolar interaction of ${ }^{131} \mathrm{Xe}$ has been advantageously used for a range of Boltzmann-polarization studies. To name just a few recent examples, ${ }^{131} \mathrm{Xe}$ has been used to investigate liquid crystals (Diehl and Jokisaari 1990b, Jokisaari 1994), porous materials (Millot et al 2001) and surfaces, using multiple quantum filtering (Meersmann et al 1998) and liquid xenon near the critical point (Pavlovskaya et al 1999). However, longitudinal relaxation times of only a few ms were observed for ${ }^{131} \mathrm{Xe}$ in lecithin vesicles at $7 \mathrm{~T}$ (Saba et al 1996). Similar and even lower values can be expected for this quadrupolar nucleus in inhomogeneous blood and tissue. Combined with the rather low concentration attainable for xenon in tissue (this being one of the main incentives for the use of hyperpolarized xenon in medicine (Albert and Balamore 1998)), the very short relaxation times are not encouraging for potential biological applications of polarized ${ }^{131} \mathrm{Xe}$.

\subsection{Diffusion}

The diffusion coefficients of gases are three to four orders of magnitude higher than those of liquids. The resolution of MR images is then limited by the typical diffusion length of the gas in the system under study (lungs, for example). For studies with hyperpolarized gases, especially with ${ }^{3} \mathrm{He}$ with high polarization $(\sim 50 \%)$ where the SNR is very high, diffusion is the main resolution-limiting factor. Knowledge of the range of diffusion coefficients to be expected is thus important for imaging experiments.

On the positive side, the large diffusion distances of gases allow a unique probe into the larger-scale structure of porous systems (Mair et al 1999, Mair and Walsworth 2002). Liquid diffusion in porous materials allows one to probe their structure on a scale below 50-100 $\mu \mathrm{m}$ and extract information about the pore size and surface to volume ratios (Butler et al 2002). However, the interconnectivity and tortuosity of the pore space, related to the transport properties of the medium, are revealed only in experiments which are able to probe longer distances. Gas diffusion, via determination of the time-dependent diffusion coefficient (Mair et al 1998, 1999), provides this complementary information. Unique insight can be gained into the structure of porous materials such as stones (Mair et al 1999) or porous glass (Moudrakovski et al 2000a, 2000b), with potential applications to human lungs. Since the diffusion coefficient of gases can be modulated by changing the (total or partial) pressure and temperature, a quite flexible range of diffusion distances can be sampled.

Thermally polarized gases have low densities and low equilibrium magnetization, making a direct determination of the diffusion coefficient (and other NMR properties) difficult under normal conditions. A diffusion coefficient of $0.051 \mathrm{~cm}^{2} \mathrm{~s}^{-1}$ was estimated for thermally polarized Xe at room temperature and atmospheric pressure from theoretical extrapolation of diffusion coefficients measured at high pressures (Peereboom et al 1989). The result was confirmed by Patyal et al (1997) using hyperpolarized xenon gas. More recently, 
Peled et al (1999) measured the diffusion coefficient of hyperpolarized Xe gas at 3 atm and $90{ }^{\circ} \mathrm{C}$ to be $D=0.0193 \mathrm{~cm}^{2} \mathrm{~s}^{-1}$. The sensitivity of the diffusion coefficient to temperature and pressure is clearly seen.

Restricted diffusion can be easily illustrated with hyperpolarized gases even at very low fields. Saam et al (1996) demonstrated edge enhancement effects due to diffusion. They use 1D imaging with hyperpolarized ${ }^{3} \mathrm{He}$ at a field of only $31 \mathrm{G}$. The size and position of the peaks which result from the edge-related effects was found to be well described by the Torrey equation with boundary conditions. Additional distortion was observed and attributed to gradients resulting from the long-range dipole fields produced by the polarized spins and by the bulk magnetic susceptibility of the glass container used in the experiments.

Song et al (1998a) use polarized ${ }^{129}$ Xe to perform a systematic study of the evolution of diffusion-mediated image distortions between the regimes of weak and strong diffusion. In the weak diffusion regime, the image distortions were found to originate from the restrictive motion near the boundaries of the sample. In the strong diffusion regime, all spins diffuse through the whole available space before dephasing from the gradient occurs. A single line is thus obtained, which can be well approximated by a Lorentzian; applying the field gradient results only in a line broadening. On the basis of these results, the authors estimate the maximum imaging resolution for xenon gas at $1 \mathrm{~atm}$ and room temperature to be about $100 \mu \mathrm{m}$ for a typical gradient strength used for microimaging.

Many applications require the use of xenon not in its gaseous phase, but rather dissolved in a variety of carriers (see section 5.2). Besides the value of $D=2.2(4) \times 10^{-5} \mathrm{~cm}^{2} \mathrm{~s}^{-1}$ for xenon in water, in good agreement to that of 'retarded' water, the diffusion of xenon dissolved in PFOB and benzene was investigated by Wolber et al with the BURST sequence. The diffusion coefficients were measured to be $D=4.5(6) \times 10^{-5} \mathrm{~cm}^{2} \mathrm{~s}^{-1}$ for xenon in PFOB and $D=12(2) \times 10^{-5} \mathrm{~cm}^{2} \mathrm{~s}^{-1}$ in benzene.

\section{NMR of hyperpolarized gases in biological systems}

\subsection{Xe in blood}

The interaction of Xe with blood is not only a crucial aspect for assessing the feasibility of Xe NMR and MRI in vivo, but also illustrative for the potential of using the sensitivity of $\mathrm{Xe}$ to its environment to probe such diverse aspects as blood oxygenation and protein structure.

Erythrocytes under normal physiological conditions have a well-defined biconcave disc shape with parameters: diameter $7.65 \mu \mathrm{m}$, maximum thickness $2.84 \mu \mathrm{m}$, minimum thickness $1.44 \mu \mathrm{m}$ and volume $97.91 \mu \mathrm{m}^{3}$ (Fung et al 1981). A mature erythrocyte contains $2.9 \times 10^{8}$ haemoglobin molecules (Plyavin and Blum 1983) which occupy close to $30 \%$ of the volume of the cell. The red blood cells in turn occupy $45-50 \%$ of the volume of whole blood. The respiratory function associated with blood is carried out by haemoglobin. On the molecular level, haemoglobin has a complex structure, consisting of around 10000 atoms, and has a molecular weight of $67000 \mathrm{~g} \mathrm{~mol}^{-1}$. Only 4 of these 10000 atoms are Fe atoms, being at the centre of the haem groups which are the binding sites for oxygen. The oxygenation state of $\mathrm{Fe}$ is the determining factor of the magnetic properties of haemoglobin.

Oxygen binding to haemoglobin proceeds via an interesting cooperative mechanism (recently reviewed by Perutz et al (1998), Eaton et al (1999)). The binding curve describing the saturation of $\mathrm{Hb}$ with oxygen as a function of oxygen partial pressure is sigmoidal instead of hyperbolic, as would be the case for a simple $\mathrm{Hb}+\mathrm{O}_{2} \leftrightarrow \mathrm{HbO}_{2}$ equilibrium. This was observed in 1904 by Christian Bohr, father of the physicist Niels Bohr, and is called the Bohr 
effect. The sigmoidal shape implies that as the number of bound oxygen molecules increases, the apparent binding affinity increases as well (by nearly a factor of 1000 for the fourth oxygen molecule compared to the first one). Since the sites are equivalent, the explanation for this striking effect lies in their interaction; binding at one site causes an effective increase in affinity at another, hence the term 'cooperative interaction'. Furthermore, the presence of carbon dioxide lowers the oxygen affinity, and $\mathrm{CO}_{2}$ binds to haemoglobin with a much larger affinity. Because of these properties, haemoglobin transports oxygen from the lungs to the tissues and carbon dioxide from the tissue to the lungs. The clarification of the haemoglobin structure, as well as the explanation for how the binding of oxygen at one site effects structural changes at the other ones and facilitates further binding, is due to Max Perutz (Perutz et al 1964, 1998, Perutz 1970).

Haemoglobin displays a pronounced susceptibility change with oxygenation, discovered by Pauling and Coryell (1936). The susceptibility of red blood cells can be determined by magnetophoresis, which relies on the separation of media with different susceptibilities using a high magnetic field gradient (only relative susceptibilities are measured). Melville, Paul and Roath were the first to demonstrate the direct magnetic separation of RBC's in their deoxygenated state from whole blood (Melville et al 1975a), and the values generally adopted were determined by Plyavin and Blum using magnetophoresis (Plyavin and Blum 1983). Spees et al (2001) recently remeasured the magnetic susceptibility of the red blood cells in different states of oxygenation, and of human blood plasma, using a SQUID magnetometer, and obtained values close to those of Plyavin and Blum. The susceptibilities for plasma, oxyhaemoglobin and deoxyhaemoglobin are, in CGS units (for SI units, an additional factor of $4 \pi$ is required $): \chi($ plasma $)=(-0.714 \pm 0.007)$ ppm, $\chi(\mathrm{CO}-\mathrm{RBC}) \simeq \chi($ oxyRBC $)=$ $(-0.749 \pm 0.010) \mathrm{ppm}$ and $\chi($ deoxyRBC $)=(-0.483 \pm 0.013) \mathrm{ppm}$. Spees et al point out that the difference in magnetic susceptibility between oxygenated and deoxygenated red blood cells is found to be $0.27 \pm 0.02 \mathrm{ppm}$, that is significantly higher than the value of $0.18-0.2$ usually used in MRI literature.

Following the ground-breaking investigation of the use of polarized xenon for the study of biological systems (rat) by Albert et al (1994), the possibility of performing NMR and MRI using hyperpolarized xenon to probe human tissue and blood was immediately considered. The survival of polarization during transport of xenon via circulation to the target organ depends critically on the spin-lattice relaxation time of ${ }^{129} \mathrm{Xe}$ in blood. Substantial effort by several groups has been dedicated to the study of the interaction between xenon and blood in vitro, with the goal of determining its effect at physiological parameters in vivo (Albert et al 1995, 1996, 1999, 2000, Bifone et al 1996, Tseng et al 1997, Wilson et al 1999a, 1999b, Wolber et al 1999a, 2000a, 2000b, 2000c, 2000d and 2002).

The behaviour of xenon in human blood was first investigated by Albert et al (1995). They were able to resolve two peaks, using thermally polarized xenon in blood, and assigned them to plasma and red blood cells, respectively. The presence of the two peaks was confirmed in a subsequent study by Bifone et al (1996).

Because of the vastly improved time resolution brought by hyperpolarized xenon, it was possible to study the dynamics of exchange processes. Bifone et al (1996) were able to observe hyperpolarized xenon penetrating the red blood cells in fresh human blood and equilibrating between the blood+saline solution within $200 \mathrm{~ms}$ after injection. The longitudinal relaxation time for xenon equilibrated in the solution was measured to be $5 \mathrm{~s}$, for both components. Previously, values of the relaxation times of $5 \mathrm{~s}$ for RBC and $10 \mathrm{~s}$ for plasma were determined in the study of Albert et al (1995). This was due to the fact that thermally polarized xenon was used, requiring a large number of averages and long measurement times, during which the $\mathrm{RBC} /$ saline mixture segregated. Furthermore, Bifone et al (1996) determined the exchange 
time for xenon between plasma and red blood cells to be $12 \mathrm{~ms}$ (comparable with the NMR time scale). The residence time constant of xenon in the RBCs was $20.4 \pm 2 \mathrm{~ms}$ and in plasma $\tau_{\mathrm{pl}}=29.1 \pm 2 \mathrm{~ms}$. Interestingly, the residence time in the RBC corresponds to a diffusion distance of around $11 \mu \mathrm{m}$ (assuming $D=10^{-5} \mathrm{~cm}^{2} \mathrm{~s}^{-1}$ ), which is significantly larger than the characteristic dimension of an $\mathrm{RBC}$ of around $8 \mu \mathrm{m}$ (see above). This impressive temporal resolution was achieved in a basically one-shot experiment (the signal from all the polarized xenon was manipulated in the first shot) by first selectively inverting the saline/plasma component and then observing the recovery of the ${ }^{129} \mathrm{Xe}$ spectrum to equilibrium by consecutive pulses with low flip angle.

The question of the influence of blood oxygenation on the NMR properties of xenon in blood arose early. As discussed in section 4.7, molecular oxygen is known to substantially reduce the $T_{1}$ of Xe (Jameson et al 1988). However, less than 1\% of the oxygen dissolved in blood is 'free' to create paramagnetic $\mathrm{O}_{2}$ centres in the plasma (Albert et al 1999). The rest $(>99 \%)$ binds to the haemoglobin of the red blood cells, resulting in the diamagnetic oxyhaemoglobin $\mathrm{HbO}_{2}$. Arterial blood contains oxygen with a partial pressure of $\mathrm{pO}_{2}=$ $95 \mathrm{mmHg}$, and venous blood has $\mathrm{pO}_{2}=40 \mathrm{mmHg}$ (Albert et al 1999). These partial pressures correspond to a degree of 95-100\% saturation of haemoglobin with oxygen for arterial blood and around $65 \%$ for venous blood. Since the two forms of haemoglobin have different susceptibilities, blood oxygenation is clearly expected to influence the chemical shift and relaxation time of the highly environment-sensitive xenon.

The longitudinal relaxation time of xenon in blood increases with increasing blood oxygenation, and this trend does not depend on field strength (Albert et al 1996, 1999, 2000, Wolber et al 1999b, 2000a, 2000b, 2000c and 2000d). The same trend was observed for a solution of lysed RBCs (solution of haemoglobin in saline). Albert et al (1999) report an increase in $T_{1}$ at $4.7 \mathrm{~T}$ from $(2.86 \pm 0.11) \mathrm{s}$ in deoxygenated solution to $(10.22 \pm 0.38) \mathrm{s}$ in the oxygenated form. For comparison, in whole blood the increase is slightly reduced: from approximately $3 \mathrm{~s}$ for both plasma and RBC in deoxygenated blood to around $8 \mathrm{~s}$ in oxygenated blood. The effect of adding carbon monoxide to blood is similar in trend but shows increased magnitude compared to that produced by oxygenating the blood. The dependence of the relaxation rate $T_{1}^{-1}$ on the relative concentration of deoxyhaemoglobin is illustrated in figure $7(\mathrm{a})$, and seen to be nonlinear.

Within a simple picture, the oxygenation dependence of $T_{1}$ might result from the decreased diamagnetism of deoxygenated compared to oxygenated haemoglobin (equivalent to an increase in paramagnetism relative to plasma) (Albert et al 1999, Cherubini and Bifone 2003). In this case, however, a linear increase in the relaxivity with the concentration of haemoglobin might be expected (Cherubini and Bifone 2003), in disagreement with the pattern seen in figure 7 . We note that the relaxation produced by susceptibility microheterogeneity is a complicated issue (Weiskoff et al 1994) and the dependence on the concentration needs not be linear, but the experimental information is not conclusive at present.

A more appealing interpretation (Albert et al 1999 and 2000, Cherubini and Bifone 2003) suggests that conformational changes induced in haemoglobin by oxygen binding might be responsible for the observed effects. The function of haemoglobin is modulated by conformational changes induced by a variety of ligands, especially oxygen and carbon monoxide. Such changes can well affect the accessibility to the xenon binding sites, or even result in xenon binding at a different site. This picture allows for the enhanced effect of carbon monoxide, since $\mathrm{CO}$ produces the same type of conformational changes as oxygen, but of larger amplitude. Furthermore, the explanation is consistent with the fact that xenon solubility in red blood cells decreases from 0.2 to 0.17 with increasing oxygenation (Clever 1979) (see table 2). 


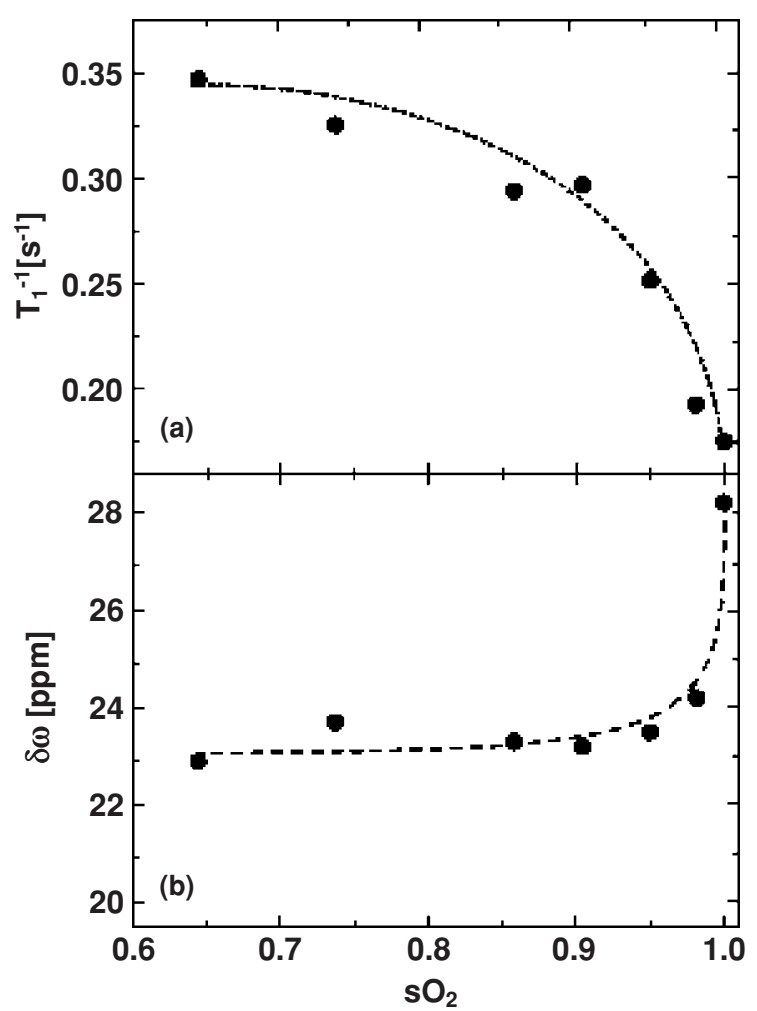

Figure 7. Longitudinal relaxation rate (a) and the separation between the plasma and RBC resonances (b) as a function of blood oxygenation level, $\mathrm{sO}_{2}$. Reproduced with permission from Cherubini and Bifone (2003) (data from Wolber (2000c)).

On the basis of the sensitivity of $T_{1}$ and the chemical shift to the level of blood oxygenation, it was suggested (Bifone et al 1999, Albert et al 1999, 2000, Wolber et al 2000a, 2000b) that xenon could be used to monitor brain activity in a quantitative way, perhaps more accurately than currently achievable with proton MRI. We note here that the dependence of the chemical shift of xenon on temperature can be very pronounced; however, it displays a minimum very close to $37{ }^{\circ} \mathrm{C}$ (Seydoux 1995). The influence of temperature variation on the accuracy of blood oxygenation measurements using Xe can, therefore, be expected to be small for in vivo studies.

The transversal relaxation of xenon in blood has been investigated by Wilson et al (1999a, $2000 \mathrm{~b})$ at $9.4 \mathrm{~T}$ and by Wolber et al (2000d) at $1.5 \mathrm{~T}$. Wilson et al report that the $T_{2}$ of the plasma resonance decreases from 5.5 to $2.5 \mathrm{~ms}$, and that of xenon in $\mathrm{RBC}$ decreases from 1.9 to $1.4 \mathrm{~ms}$ from oxygenated to deoxygenated blood. At $1.5 \mathrm{~T}$, Wolber et al determine that $T_{2}$ is oxygenation independent. The results are presumably due to the rapid xenon chemical exchange between the bound phase and the solution, resulting in a field-dependent transverse relaxation time (Cherubini and Bifone 2003).

\subsection{Delivery}

The simplest administration route is via the respiratory system. Since the overwhelming majority of applications of ${ }^{3} \mathrm{He}$ to living systems concern the lungs, respiratory 
delivery is used nearly exclusively for in vivo ${ }^{3} \mathrm{He}$ studies and has been used for xenon as well. For humans, inhalation from a Tedlar bag is most common (Venkatesh et al 2003, Bidinosti et al 2003, Kilian 2001, Mugler et al 1997). For animals, systems with different degrees of sophistication exist, up to dedicated small animal ventilators (Swanson et al 1997, Rosen et al 1999, Venkatesh et al 1999, Hedlund et al 2000).

Compartmental models have been used to predict the concentration of hyperpolarized xenon (which is soluble in tissue, in contrast to helium) in human tissues following the breathing of the gas (Peled et al 1996, Martin et al 1997, Lavini et al 2000). Because of the high sensitivity of xenon's chemical shift to its environment (section 4.5), the distribution in different tissues can be determined experimentally by chemical shift imaging (Swanson et al 1999).

For delivery to distal organs, however, the shorter way and presumably the path of least relaxation, is via the circulatory system directly (injection). The line corresponding to $\mathrm{Xe}$ in the delivery medium should ideally be: (a) narrow, to allow study of the dynamics of the delivery process; and (b) not obscure lines from xenon in tissue or blood. Xenoncarrier agents investigated so far include saline (Bifone et al 1996, Goodson et al 1997, Zhao et al 1998), intralipid solution (Goodson et al 1997, Zhao et al 1998, Wolber et al 1999b, Möller et al 2002, Duhamel et al 2000, 2001), and perfluorocarbon solutions (Goodson et al 1997, Zhao et al 1998, Wolber et al 1999b).

The longitudinal relaxation time of ${ }^{129} \mathrm{Xe}$ in saline is quite long, and even longer in deuterated water (66 and $\sim 1000 \mathrm{~s}$, respectively (Bifone et al 1996)), but the solubility is relatively poor, with an Ostwald coefficient of only $\sim 0.09$. Alternatively, Intralipid solution (a suspension of lipid vesicles in water) profits from the high solubility of xenon in lipids and displays moderate relaxation, with $T_{1}$ between 40 and $50 \mathrm{~s}$ (Bifone et al 1996, Zhao et al 1998). An even higher solubility, of $\sim 1.2$, is observed for xenon in perfluorocarbon compounds, extensively used as blood substitute (Wolber et al 1999b), and the long $T_{1}$ is also advantageous (94 s, (Zhao et al 1998)). However, the rapid exchange between the perfluorocarbon compartment and aqueous phase increases the ${ }^{129} \mathrm{Xe}$ NMR line-width substantially, which has been shown to depend on the size distribution of the droplets in the emulsion (Wolber et al 1999b). Narrower line widths are observed in perfluorocarbon emulsions with larger droplets $(>5 \mu \mathrm{m})$.

Delivery of the hyperpolarized gas in gas-filled microbubbles or liposomes has been considered recently and can be applied also to helium (Chawla et al 1998, 2000, Callot et al 2001a, 2001b, Venkatesh et al 2000, 2001, Duhamel et al 2001). The $T_{1}$ for ${ }^{129} \mathrm{Xe}-$ filled liposomes ranges from $116 \mathrm{~s}$ in vitro to $54 \mathrm{~s}$ in vivo at $4.7 \mathrm{~T}$ (Venkatesh et al 2000) and a narrow line was observed. This delivery agent seems very well suited for angiography applications.

Duhamel et al (2001) compared the $T_{1}$ values of hyperpolarized ${ }^{129} \mathrm{Xe}$ dissolved in lipid emulsions and xenon microbubbles ( 2 to $3 \mu \mathrm{m}$ in diameter) at $2.35 \mathrm{~T}$. The NMR spectrum of ${ }^{129} \mathrm{Xe}$ dissolved in the intralipid solution presents only one peak (194 ppm), with $T_{1}$ of $21 \mathrm{~s}$ (30\% Intralipid). The spectrum of ${ }^{129} \mathrm{Xe}$ in the microbubbles displays both a strong gas-phase resonance $(0 \mathrm{ppm})$ and the peak of dissolved xenon $(204 \mathrm{ppm})$, with a 4:3 ratio. Relaxation times of 20 and $19 \mathrm{~s}$ were observed for the gas and dissolved phase, respectively. At practically equal relaxation times, Intralipid is preferred over microbubbles for in vivo experiments because the signal is concentrated in a single resonance. By injecting hyperpolarized ${ }^{129} \mathrm{Xe}$ dissolved in Intralipid $30 \%$ in a rat carotid, Duhamel et al $(2000,2001)$ were able to perform blood-flow measurements and perfusion imaging of the rat brain with a high SNR. 


\section{Specific MRI techniques}

The choice of MRI technique for a given problem is very much determined by the details of the particular question; choice of sequence for cardiac imaging is largely determined by the need to suppress, or eliminate motion-induced artefacts. In the case of hyperpolarized Xe, there are two important and overriding factors: (a) the hyperpolarization is non-renewable; and (b) the hyperpolarization has to reach the target of interest before it can be imaged. The latter implies that a sufficient amount must arrive in order for successful imaging or spectroscopy to be performed. The first consideration dictates either single-shot methods such as echo planar imaging, RARE, or low flip angle techniques such as FLASH. A further, and more immediate, consequence of the non-renewable nature of the hyperpolarization is that all scanner adjustments must be made either on an additional phantom or on only a very small fraction of the hyperpolarization. The second consideration normally dictates a rapid acquisition too but additionally requires the use of some form of carrier to 'protect' the hyperpolarization from magnetic impurities and relaxation centres. An excellent overview of MRI techniques in hyperpolarized imaging has been given by Zhao et al (1998).

The gradient echo sequence has been used in much of the work with hyperpolarized Xe because of its simplicity. Zhao et al (1996) examined issues related to optimization of the gradient echo sequence for imaging of hyperpolarized Xe. In particular, they investigated the use of variable flip angles (VFA) for the radiofrequency excitation and compared them to the use of constant flip angle (CFA) excitation. The exact nature of the traversal through k-space also has very important implications for the quality of the final image. The authors compare centrically and sequentially ordered acquisitions of the k-space data. The choice of flip angle, sampling order, and resolution have significant consequences for the final image. The variable flip angle approach was shown to improve the SNR and eliminate some image artefacts. The impetus for the use of variable flip angles for radiofrequency excitation arises as a straightforward consequence of the fact that each pulse depletes the magnetization available for the next one and that the hyperpolarized magnetization will not be replenished through normal $T_{1}$ relaxation, that is, the hyperpolarization in non-renewable. Experimental data and simulations were presented to show that if constant flip angles are employed then the magnitude of the excited transverse magnetization decreases with each excitation and this, through a poorer point spread function, has consequences for the spatial resolution of the image. By using an optimized scheme where the first flip angle is small and the final one is $90^{\circ}$, the magnitude of the excited transverse magnetization was shown to be approximately independent of excitation number. Bearing in mind the long $T_{1}$ relaxation time of hyperpolarized Xe, the authors derived the following expression for calculating the flip angle of the $n$th pulse, $\theta_{n}$, to maintain a constancy of the signal:

$$
\theta_{n}=\sin ^{-1}\left[\frac{\exp \left[(n-1) T_{\mathrm{R}} / T_{1}\right] \sin \theta_{1}}{\Pi_{j=1}^{n-1} \cos \theta_{j}}\right],
$$

where $T_{\mathrm{R}}$ is the repetition time of the gradient echo sequence $\theta_{1}-T_{\mathrm{R}}-\cdots-\theta_{n}-T_{\mathrm{R}}-\cdots-\theta_{N}$ and $\theta_{N}=90^{\circ}$.

The constant and variable flip angle approaches were modelled using phase profiles along the length of a hyperpolarized Xe gas cell with a rectangular profile to highlight the relative advantages of the two approaches. A comparison of the CFA approach with sequential and centric ordering with the VFA approach with sequential ordering showed the latter to be preferable; in the sequential VFA method the shape of the profile was much closer to that expected from a rectangular sample. Experimental profiles were shown as confirmation of the simulation results. Images of hyperpolarized Xe in a cylinder were also acquired with (a) CFA 
and sequential ordering, (b) CFA with centric ordering and (c) VFA with sequential ordering. The CFA image with sequential ordering showed the lowest SNR. This is to be expected since by the time the centre of $\mathrm{k}$-space is reached, the magnetization has been considerable depleted by the preceding pulses. The CFA approach with centric ordering showed much improved SNR but the edges of the cylinder were blurred as found in the simulations of the profiles. The VFA approach with sequential resulted in an image with considerably improved homogeneity and sharper edges.

\subsection{SNR considerations}

Hoult and Richards (1976) deduced an expression for the NMR signal detected in a pickup coil, given by

$$
S=\left(B_{\mathrm{r}} / I_{\mathrm{r}}\right) V_{\mathrm{s}} \omega_{0} M_{0},
$$

where $B_{\mathrm{r}} / I_{\mathrm{r}}$ is the magnetic field per unit current for the receiver coil, $V_{\mathrm{s}}$ is the volume of the sample and $\omega_{0}=\gamma B$ is the Larmor frequency. $M_{0}$, the magnetization density, is expressed as

$$
M_{0}=P N_{\mathrm{s}} \gamma I
$$

where $N_{\mathrm{s}}$ is the spin density, $P$ is the degree to which the spins are polarized, and $\gamma$ characterizes the magnetic moment of the individual spin $I$ ( $1 / 2$ in our case).

Combining equations (12) and (13) results in a signal dependence of $S \sim \gamma^{2} B_{0} P$. The main source of noise at low field is Johnson noise from the receiver coil at temperature $T$; this is also the case for a sufficiently small sample at high field. The Johnson noise $N$ can be expressed as

$$
N=\left(4 k_{\mathrm{B}} T \cdot R \cdot \Delta f\right),
$$

with $\Delta f$ being the bandwidth of the system, and $R$ the resistance of the coil. $R$ depends weakly on the frequency, due to the RF skin depth of the coil: $R \sim \omega^{1 / 4} \sim(\gamma B)^{1 / 4}$. In large (coil filling) biological samples at high field, the dominant source of noise comes from the sample. Inductive coupling of the noise from the weakly conducting sample with the coil leads to a field dependence of the noise $N \sim \omega \sim \gamma B$.

The SNR therefore shows a field dependence:

$$
\mathrm{SNR} \sim\left(\gamma^{2} B_{0} P\right) /\left(\gamma B_{0}\right)^{1 / 4}=\gamma^{7 / 4} B_{0}^{3 / 4} P,
$$

for Johnson noise (either low field or high field and small sample) or

$$
\mathrm{SNR} \sim\left(\gamma^{2} B_{0} P\right) /\left(\gamma B_{0}\right)=\gamma \cdot P,
$$

for sample-dominated noise (high field, large sample).

For hyperpolarized gases $P$ is independent of the applied field. The Boltzmann (equilibrium, thermal) polarization is given by equation (1) and depends on $\gamma B_{0}$. If we denote $\mathrm{SNR}_{\text {pol }}$ and $\mathrm{SNR}_{\text {th }}$ the signal-to-noise-ratio of a hyperpolarized $\left(\mathrm{Xe},{ }^{3} \mathrm{He}\right.$ ) and a thermally polarized sample (water, for example), respectively, the field dependence of the ratio $\mathrm{SNR}_{\mathrm{pol}} / \mathrm{SNR}_{\mathrm{th}}$ is

$$
\mathrm{SNR}_{\mathrm{pol}} / \mathrm{SNR}_{\mathrm{th}} \sim 1 / B_{0} .
$$

It is easily seen from equation (17) that, even if from the SNR point of view imaging with hyperpolarized gases offers a moderate advantage over imaging with thermally polarized protons at high field (e.g. due to gas-liquid density difference of roughly four orders of magnitude), this changes into a tremendous advantage at very low field. For example, even assuming an $\mathrm{SNR}_{\mathrm{pol}} / \mathrm{SNR}_{\text {th }}$ of only 1 at a field of $1 \mathrm{~T}$, it becomes an overwhelming $10^{4}$ at a field of $1 \mathrm{G}$. Further low-field advantages are discussed in section 9. Of course, the usefulness of hyperpolarized gases is not limited to low-field applications. Lung imaging, for which polarized ${ }^{3} \mathrm{He}$ is extensively used, is very difficult with conventional MRI. 


\subsection{Resolution}

The natural linewidth of an NMR line is given by the transverse relaxation time $T_{2}$. This linewidth provides an absolute limit to the spatial resolution which can be obtained by encoding the frequency of the sample. It is narrowest for rapidly tumbling molecules, such that the best resolution is obtained in liquids. Characterizing a given sample by one transverse relaxation time $T_{2}$ assumes that the sample is homogeneous and all the spins precede at the same Larmor frequency $\gamma B_{0}$. Furthermore, since spatial encoding is obtained by applying gradient fields for a given period of time, for optimum resolution the spins should be 'freezed' at the point where encoding takes place. The two conditions are seldom fulfilled in practice, and two samplerelated effects, susceptibility heterogeneity and diffusion, limit the attainable resolution. For an in-depth discussion of resolution in MRI (see, e.g., Callaghan (1991)).

In the presence of field inhomogeneities, the local frequency changes to $\omega(\mathbf{r})=$ $\omega_{0}+\gamma \Delta B(\mathbf{r})$. This results in a line broadening, additional decoherence is caused by the mismatched frequencies, and the effective contribution to $T_{2}$ reads

$$
1 / T_{2}^{*}=1 / T_{2}+1 / 2 \gamma \Delta B
$$

This is the case whenever the applied field or the sample (or both) show inhomogeneities. The inhomogeneity of the applied field can be corrected by superimposing magnetic fields produced by additional coils (shimming). The inhomogeneity of the sample which is detrimental to imaging is the susceptibility heterogeneity, $\Delta \chi$, which produces field inhomogeneities $\Delta B=\Delta \chi \cdot B_{0}$.

The spatial information required to produce images from the NMR signal is provided by encoding gradients, $\mathbf{G}=G_{x} \mathbf{x}+G_{y} \mathbf{y}+G_{z} \mathbf{z}$. By keeping gradients on for a period of time, before and/or at the time of detection, phase and/or frequency encoding are provided, respectively. In the simplest case, the NMR signal is detected in the presence of a gradient (read-out gradient) and the spatially encoded frequency of the signal becomes: $\omega(\mathbf{r})=\omega_{0}+\gamma \cdot \Delta \chi(\mathbf{r}) \cdot B_{0}+\gamma\left(G_{x} x+G_{y} y+G_{z} z\right)$. It is clear that the susceptibility heterogeneity of the sample leads to image distortions by providing an additional positiondependent term. The effect of this term can be minimized either by increasing the gradient strength, or by reducing the field.

The attainable resolution is further influenced by diffusion. The signal attenuation due to diffusion of nuclei in an applied field gradient has the expression $\exp \left(-1 / 3 \gamma^{2} G^{2} D t^{3}\right)$, where $D$ is the diffusion coefficient (Callaghan 1991). It is equivalent to a contribution to $1 / T_{2}^{*}$ of $1 / 3 \gamma^{2} G^{2} D \tau^{2}$, with $\tau$ related to the echo time used by the imaging sequence. For nuclei diffusing in an inhomogeneous field produced by the sample susceptibility heterogeneity, a similar expression is obtained in a linear approximation,

$$
1 / T_{2 \text { diff }}^{*}=1 / 3\left[\gamma(\Delta \chi / l) B_{0}\right]^{2} D \tau^{2} .
$$

Here $l$ is a characteristic length over which the mean susceptibility varies by $\Delta \chi$. This expression corresponds to a 'slow diffusion' regime, where the characteristic diffusion time is long compared to the frequency shifts induced by the varying susceptibility, $\left(\gamma(\Delta \chi / l) B_{0}\right)\left(l^{2} / D\right) \gg 1$. In a fast diffusion regime, where $\left(\gamma(\Delta \chi / l) B_{0}\right)\left(l^{2} / D\right) \ll 1$, the contribution to the linewidth is given by

$$
1 / T_{2 \text { diff }}^{*}=1 / 3\left[\gamma(\Delta \chi) B_{0}\right]^{2}\left(l^{2} / D\right)
$$

Both expressions, equation (19) as well as equation (20), show a $B_{0}^{2}$ dependence. Lowfield imaging clearly minimizes the loss of resolution (as well as signal intensity) due to susceptibility inhomogeneity, and should be used preferentially whenever the sample is very heterogeneous (lungs, for example). For a more detailed discussion see Wong et al (1999). Low-field applications are reviewed in section 9. 


\section{MRI results}

Several reviews have been written about in vivo MRI using hyperpolarized gases (Möller et al 2002, Kauczor et al 2002). Here, some of the newer and important results are reviewed. The first experiments with hyperpolarized ${ }^{129} \mathrm{Xe}$ on humans were reported by Albert et al (1994) where they imaged ${ }^{129} \mathrm{Xe}$ in the gas phase and demonstrated images of the human oral cavity. In this particular report they noted the range of frequencies of ${ }^{129} \mathrm{Xe}$ in lipids, proteins and water-a range of $20 \mathrm{ppm}$. Experiments were performed using a conventional 2D gradient echo sequence with a flip angle of $10^{\circ}$ and a TR of $300 \mathrm{~ms}$. In a subsequent publication, Mugler et al (1997) reported preliminary imaging and spectroscopic experiments in humans. Using polarization fractions in the range of $0.1-2 \%$, their head spectra showed one peak from the gas phase and one prominent peak from the dissolved phase; again time-resolved spectra were obtained. Following a period of inhalation, from the integrated intensity of the dissolved peak they suggested that the ${ }^{129} \mathrm{Xe}$ signal in brain reaches a maximum $5 \mathrm{~s}$ after and then decays to zero in about $50 \mathrm{~s}$. These results are consistent with the known transit time from the lungs to the brain which is of the order of $5 \mathrm{~s}$. The lack of a 'red blood cell' peak in their results was explained by a combination of rapid uptake of ${ }^{129} \mathrm{Xe}$ by brain parenchyma and the small volume fraction of blood in the brain resulting in a blood signal level which fell below the noise for the given experimental conditions. Although the reported experiments were carried out under sub-optimal conditions, the authors calculated the achievable improvements and suggested that dissolved phase imaging of the brain with a resolution similar to current gas-phase images of the lungs might be possible.

The early euphoria surrounding the possible in vivo applications of hyperpolarized ${ }^{129} \mathrm{Xe}$ has given way to careful and thorough experiments often using spectroscopic techniques. Recently, Kilian et al (2004a) have investigated human brain perfusion by acquiring several time series of ${ }^{129} \mathrm{Xe}$ spectra from a healthy volunteer after inhalation of hyperpolarized gas. They analysed the time-dependent amplitudes of the MR spectra using a compartment model for xenon uptake which had been modified to account for the loss of polarization due to radiofrequency excitation and for the breathhold technique that was employed. From their analysis, they suggest that the resonances detected at $196.5 \pm 1 \mathrm{ppm}$ and $193 \pm 1 \mathrm{ppm}$ originate from Xe dissolved in grey and white matter, respectively. Furthermore, they measured the $T_{1}$ relaxation times of Xe and found a longer $T_{1}$ in grey matter than in white matter.

Hyperpolarized Xe has also been used to explore lung function-normally the domain of hyperpolarized ${ }^{3} \mathrm{He}$. Here, Ruppert et al (2004) have turned the 'disadvantage' that Xe dissolves readily into an advantage to quantitate gas exchange in the lung. They employed spectroscopy and imaging techniques in animal models to investigate the dependence of the relative xenon gas exchange rate on the inflation level of the lung and the tissue density. Their results indicate that gas exchange occurs on a timescale of milliseconds, with an average effective diffusion constant of about $3.3 \times 10^{-6} \mathrm{~cm}^{2} \mathrm{~s}^{-1}$ in the lung parenchyma. Using polarization-transfer imaging pulse sequences, they were able to detect regionally increased gas-exchange rates in the lung, indicative of increased tissue density secondary to gravitational compression.

Animal experiment performed by Mansson et al (2003) have yielded values for structural (average alveolar wall thickness) and perfusion-related parameters (pulmonary perfusion, capillary diffusion length and mean transit time) which are in agreement with values obtained from non-NMR methods. In comparisons between healthy rats and rats with acute inflammatory lung injury they found significant differences in diffusion length (control $8.5 \pm 0.5 \mu \mathrm{m}$, injured $9.9 \pm 0.6 \mu \mathrm{m}, P<0.001$ ), in capillary diffusion length (control $2.9 \pm$ $0.4 \mu \mathrm{m}$, injured $3.9 \pm 1.0 \mu \mathrm{m}, P<0.05$ ), and in pulmonary haematocrit (control $0.55 \pm 0.06$, 
injured $0.43 \pm 0.08, P<0.01)$. However, no differences were observed in alveolar wall thickness, pulmonary perfusion, and mean transit time. These results demonstrate the ability of the MRI with hyperpolarized Xe to investigate two main aspects of lung function.

Recent results presented by several groups at the conference of the International Society of Magnetic Resonance in Medicine in 2004 show much promise. Functional brain imaging using hyperpolarized Xe has been reported in the rat brain (Mazzanti et al 2004). The brains of male Sprague-Dawley rats were imaged whilst breathing alternative breaths of hyperpolarized Xe and an $\mathrm{O}_{2}$ /isoflorane mixture over a period of 4 min during which a so-called baseline chemical shift image was acquired. Functional haemodynamic changes were evoked pharmacologically by the administration of $5 \% \mathrm{CO}_{2}$. The animals were ventilated for a period of 5 min with a mixture of $\mathrm{O}_{2}: \mathrm{CO}_{2}$ :isoflorane (92\%:5\%:3\%) and subsequently a second chemical shift image was acquired. The two chemical shift images were subtracted to produce difference images that reflected changes in the Xe distribution resulting from breathing $\mathrm{CO}_{2}$. The authors note that the difference in the Xe distribution is likely to reflect areas of increased cerebral blood flow resulting from $\mathrm{CO}_{2}$-induced vasodilation.

Preliminary human results investigating the apparent diffusion coefficient of Xe in the lung have been presented by Mugler et al (2004b). The length scales which can be probed using Xe diffusion should be significantly smaller than those probed with ${ }^{3} \mathrm{He}$, due to the much reduced self-diffusion coefficient (factor of $\sim 30$ ). Novel information is expected to be obtained about disease processes in the lung. Hyperpolarized Xe imaging was performed on two healthy volunteers using a whole-body MRI machine and a flexible Xe chest coil. Contiguous diffusion images, which were obtained covering the whole lung using a FLASH-based sequence, were used to calculate the apparent diffusion coefficient (ADC) on a voxel-by-voxel basis. The ADC values for the two subjects were $0.039 \pm 0.016 \mathrm{~cm}^{2} \mathrm{~s}^{-1}$ and $0.036 \pm 0.014 \mathrm{~cm}^{2} \mathrm{~s}^{-1}$. Apart from the expected lower spatial resolution, the ventilation images were qualitatively similar to those obtained from healthy volunteers using ${ }^{3} \mathrm{He}$, that is, the Xe signal was reasonably uniformly distributed throughout the lung parenchyma. Similarly, the ADC maps showed fairly uniform values throughout the lung and these values were approximately five to six times smaller than those of ${ }^{3} \mathrm{He}$.

Kilian et al (2004b) have demonstrated temporally- and spatially-resolved in vivo spectroscopy of the human brain using hyperpolarized Xe. The main improvements here resulted from the use of a commercial Xe head coil and the measurements were performed at $3 \mathrm{~T}$. In the 2D CSI measurements, inhalation of hyperpolarized Xe was started $20 \mathrm{~s}$ before the sequence. The time-dependent spectroscopic measurements demonstrated two resonance lines, one at $196 \mathrm{ppm}$ and the other at $193 \mathrm{ppm}$, in keeping with previously reported results. The dynamics of the two peaks were demonstrated over a period of about $100 \mathrm{~s}$. With increased hyperpolarization fractions and improved data analysis, the method should enable the investigation of perfusion changes in the human brain.

\section{Polarization transfer}

In 1996, Navon et al discovered that hyperpolarized Xe can transfer its polarization to the protons of liquid benzene (Navon et al 1996). This effect, termed spin polarized induced nuclear Overhauser effect (SPINOE), is based on Xe-H dipolar cross-relaxation and is a novel manifestation of the well studied nuclear Overhauser effect (NOE) (Overhauser 1953, Solomon 1955). The measured fractional enhancement factor, $f=\left(I-I_{0}\right) / I_{0}$, of the ${ }^{1} \mathrm{H}$ magnetization amounted to approximately 0.1 for Xe in benzene at $4.2 \mathrm{~T}$ and at $300 \mathrm{~K}$ (Navon et al 1996). 
$I$ is the enhanced proton magnetization and $I_{0}$ the proton Boltzmann magnetization at thermal equilibrium. Slightly higher enhancements were obtained with partially deuterated samples, where the ${ }^{1} \mathrm{H}$ autorelaxation was suppressed.

The effect has generated some of the most interesting applications involving hyperpolarized xenon. A number of techniques have been used over the last decade to induce and study polarization transfer from hyperpolarized Xe to protons and other nuclei such as 13C, 29Si. They include low field thermal mixing, high-field cross-polarization, dissolution in liquid and supercritical xenon, and, most broadly used, the spin polarization-induced nuclear Overhauser effect (SPINOE) (Driehuys et al 1993, Long et al 1993, Driehuys et al 1995, Rõõm et al 1997, Augustine and Zilm 1997, Song et al 1997, Pietraß et al 1998, Brunner et al 1998a, 1998b, Fitzgerald et al 1998, Sauer et al 1999, Luhmer et al 1999, Stith et al 1999, MacNamara et al 1999, Leawoods et al 2000, Appelt et al 2001). SPINOE is easiest to perform experimentally and has so far proved to be the most generally applicable technique.

The subject of polarization transfer from polarized xenon to other nuclei is the exclusive subject of one excellent review (Song 2000) and an important part of a few excellent others (Pietraß 1999, Brunner 1999a, 1999b, Goodson 2002). We discuss it only briefly in the following, with focus on SPINOE and thermal mixing.

\subsection{SPINOE}

The Solomon equations for the evolution of the magnetization of the two spin ensembles coupled by the dipolar interaction are (Solomon 1955):

$$
\begin{aligned}
& \frac{\mathrm{d} \bar{I}}{\mathrm{~d} t}=-\rho_{I}\left(\bar{I}-I_{0}\right)-\sigma\left(\bar{S}-S_{0}\right) \\
& \frac{\mathrm{d} \bar{S}}{\mathrm{~d} t}=-\rho_{S}\left(\bar{S}-S_{0}\right)-\sigma\left(\bar{I}-I_{0}\right),
\end{aligned}
$$

where the bar indicates an ensemble average, and the label 0 denotes equilibrium (thermal) polarization. These equations show that, whenever the magnetization of one of the interacting spin species is different from the equilibrium value, not only does it evolve back towards equilibrium (process governed by the self-relaxation rate $\rho=1 / T_{1}$ ), but it also affects the magnetization of the other species via the cross-relaxation rate, $\sigma$. The effect was predicted by Overhauser (1953) and confirmed experimentally by Carver and Slichter (1956) for metals. The magnetization transferred from electrons to nuclei results in an enhancement factor of three orders of magnitude ( $\gamma_{e}$ is around 1000 times larger than that of the proton).

In a similar manner, polarization transfer from hyperpolarized xenon or helium to other nuclear species (protons, most often) can be expected. For the steady state, the Solomon equations (Solomon 1955) lead to the expression for the fractional enhancement, $\epsilon$, of the magnetization of a nuclear species $\mathrm{H}$ (protons, but valid for any other nucleus):

$$
\epsilon=\frac{\gamma_{\mathrm{Xe}}}{\gamma_{\mathrm{H}}} \cdot \frac{\sigma_{\mathrm{H}-\mathrm{Xe}}}{\rho_{\mathrm{H}}} \cdot \frac{S-S_{0}}{S_{0}},
$$

where $\gamma_{\mathrm{Xe}}$ and $\gamma_{\mathrm{H}}$ are the gyromagnetic ratios of the $\mathrm{Xe}$ and $\mathrm{H}$ nuclei, respectively, $\sigma_{\mathrm{H}-\mathrm{Xe}}$ is the $\mathrm{Xe}-\mathrm{H}$ cross-relaxation rate and $\rho_{\mathrm{H}}=1 / T_{1 \mathrm{H}}$ is the autorelaxation rate of the protons. $S$ is the enhanced Xe nuclear magnetization, $S_{0}$ is the Xe magnetization at Boltzmann equilibrium. In the case of hyperpolarization, steady state is only obtained with continuous flow (with application to surface studies, for example). A better approach for most applications is 
obtained by looking at the dynamic evolution of the magnetization of the two species he steady state. This is beyond the scope of this review. For a more in-depth discussion see, for example, the review by Song (2000).

It may be seen from equation (23) that the fractional enhancement is inversely proportional to the applied field. For example, in low field at $B_{0}=0.001 \mathrm{~T}, S_{0} \ll S$ and the ratio $S / S_{0}=P_{\mathrm{Xe}} / P_{\mathrm{Xe} 0} 10^{-1} / 10^{-9}$ is of the order of $10^{8}$. For xenon dissolved in proton-containing solutions, the ratio $\sigma_{\mathrm{H}-\mathrm{Xe}} / \rho_{\mathrm{H}}$ is typically in the range between $10^{-8}$ and $10^{-5}$, thus fractional proton enhancements where $1<\epsilon<1000$ can be expected.

Dimitrov et al (2000) investigated theoretically and experimentally the cross-relaxation of xenon dissolved in water to protons. Cross-relaxation to the protons is determined to be an important factor of relaxation of xenon in solution, and contributes up to $85 \%$ of the relaxation rate. Nevertheless, the estimated enhancement of the polarization of the water protons due to contact with 10 bar of $10 \%$ hyperpolarized xenon at moderate applied magnetic fields is minuscule, only $10^{-2}$.

Substantial enhancement of proton polarization in other solvents than water has been observed, however, by Appelt et al (2001) at low field. The observed proton NMR signal of liquid ethanol, butanol and toluene showed an enhancement at room temperature of about 1000 in a field of $B=0.001$ T. Smaller proton enhancement factors between 10 and 100 are measured for ethanol/water mixtures and for sunflower oil. Recently, Heckman et al (2003) report enhancements of the proton polarization of up to $10^{6}$ from xenon SPINOE in an ultralow field of $1 \mu \mathrm{T}$. Vastly enhanced signals from the protons of cyclopentane, acetone and methanol can also be detected (see also the following section).

The transfer of polarization from xenon to a cage molecule, $\alpha$-cyclodextrin, by SPINOE, was studied by several groups (Desvaux et al 2003, 2000, Song et al 1997) and the first proton-enhanced spectrum of a protein was recently reported (Landon et al 2001).

Techniques based on dissolving samples in liquid (Fitzgerald et al 1998, Appelt et al 2001) or supercritical xenon (Haake et al 1998, Leawoods et al 2000) can also produce sizeable signal enhancements. Following this approach, Fitzgerald et al (1998) demonstrated enhancements of over 45-fold in the proton spectra of toluene dissolved in hyperpolarized liquid xenon. It was furthermore shown that cross-relaxation with hyperpolarized liquid xenon can also provide differential enhancements of non-equivalent spins on the same molecule in solution, with the toluene proton spectrum showing a preferential enhancement of the aromatic group over the methyl protons.

Smith et al (2002) compared SPINOE with cross-polarization (through dipolar coupling) from spin-polarized xenon on simple silica surfaces, to assess sensitivity and selectivity enhancement in surface studies using solid-state NMR. Lineshape analysis of ${ }^{1} \mathrm{H}$ spectra for CP and SPINOE experiments demonstrates the difference in selectivity of methods due to longer SPINOE evolution times that lead to greater spin diffusion. SPINOE provides significantly larger enhancements at temperatures below $160 \mathrm{~K}$, while $\mathrm{CP}$ performs well at higher temperatures, is more selective to surface nuclei, and has the advantage of short acquisition delays.

\subsection{Thermal mixing}

The first successful experiments showing signal enhancement of other nuclei due to polarized ${ }^{129} \mathrm{Xe}$ used low-field thermal mixing $\left({ }^{131} \mathrm{Xe}\right.$ in xenon ice, protons in a silicone coating, carbon in ${ }^{13} \mathrm{CO}_{2}$ (Gatzke et al 1993, Driehuys et al 1993, Bowers et al 1993)). Polarization transfer to the spin $3 / 2$ isotope ${ }^{131} \mathrm{Xe}$ was identified by Gatzke et al (1993) as being the main relaxation 
mechanism of ${ }^{129} \mathrm{Xe}$ in $\mathrm{Xe}$ ice at low temperatures (close to $4.2 \mathrm{~K}$ ) and low-fields (a few hundred $\mathrm{G}$ for temperatures of tens $\mathrm{K}$ and even up to $1 \mathrm{kG}$ at $4.2 \mathrm{~K}$ ). Cross-relaxation is the consequence of degeneracy of the energy levels of the two magnetic species and the presence of dipolar interactions between them. Since ${ }^{131} \mathrm{Xe}$ has a magnetic moment which is nearly four times smaller than that of ${ }^{129} \mathrm{Xe}$, the degeneracy arises from the extra energy provided by quadrupole interaction. The nuclear quadrupole moment interacts with the electric field gradient created by imperfections in the lattice, or near boundaries of ice crystal grains. Similar results were obtained by TonThat et al (1997) for fields down to $2 \mathrm{mT}$; the differences between their measured $T_{1}$ values and those measured by Gatzke et al (1993) were assigned to the variability of the relaxation producing centres. A recent study by Lang et al (2002) showed that significant increase in the spin-lattice relaxation time of ${ }^{129} \mathrm{Xe}$ can be achieved in xenon ice, if the solid is annealed at an appropriate temperature. For the experimental conditions of $180 \mathrm{G}$ and $4.2 \mathrm{~K}$, an increase in $T_{1}$ from 20.4 to $35 \mathrm{~h}$. was demonstrated following annealing for $10 \mathrm{~min}$ at $113 \mathrm{~K}$.

The experimental conditions for thermal mixing are, however, difficult. Fields of the order of $\mathrm{mT}$ are required for efficient mixing (depending on the magnitude of the dipolar coupling), the relaxation times strongly decrease at these field values producing loss of polarization, such that the method is not adequate for substances with short relaxation times, the rapid transfer of the probe from and to the magnet is not suited for some experiments (for example, in vivo). A further serious concern is the reproducibility of the conditions and thus of the polarization transfer.

A special role in the study of biological systems is held by ${ }^{13} \mathrm{C}$, since life on Earth is carbon-based. Contrary to the ubiquous ${ }^{12} \mathrm{C}$, the odd isotope is NMR active, although with a rather low gamma (very close to that of ${ }^{129} \mathrm{Xe}$ and ${ }^{23} \mathrm{Na}$ ). The ${ }^{13} \mathrm{C}$ spectrum of chemical shifts is roughly $200 \mathrm{ppm}$ in compounds. Its generally long longitudinal relaxation time amounts to around $20 \mathrm{~s}$ in vivo (Golman et al 2003), making this nucleus a promising candidate for a number of potential MR applications. The main difficulty in performing ${ }^{13} \mathrm{C}-\mathrm{NMR}$ of biological systems is due to its low natural abundance (1.1\%). The possibility of using hyperpolarized xenon for polarization transfer to $13 \mathrm{C}$ is therefore particularly exciting.

This has been achieved with low-field thermal mixing (Bowers et al 1993), as well as with SPINOE (Fitzgerald et al 1998). In the latter study, carbon disulphide was dissolved in hyperpolarized liquid xenon. An enhancement factor of 74 was determined for ${ }^{13} \mathrm{C}$ compared to the signal obtained with thermal polarization at $200 \mathrm{~K}$ and $1.4 \mathrm{~T}$. Using dynamic nuclear polarization of ${ }^{13} \mathrm{C}$ (also of ${ }^{15} \mathrm{~N}$ ) an enhancement factor in excess of $10^{4}$ over thermal polarization has been reported by Ardenkjaer-Larsen et al (2003). Dynamic polarization is performed at temperatures around $2 \mathrm{~K}$ and high magnetic fields. Following heating and injection of the substance containing polarized ${ }^{13} \mathrm{C}$, imaging of vasculature has already been performed in vivo, on rats (Golman et al 2003). An alternative method of producing polarized ${ }^{13} \mathrm{C}$ is by use of parahydrogen induced polarization (PHIP) (Stephan et al 2002). Enhancements of up to $10^{4}$ relative to thermal polarization in a field of $1.5 \mathrm{~T}$ have been reported in vivo, and used to generate a subsecond angiogram of an animal (Golman et al 2003).

Recently, Cherubini et al (2003) have compared the merits of SPINOE and low-field thermal mixing for the production of hyperpolarized ${ }^{13} \mathrm{C}$ by using optically pumped xenon. The observed values of the enhancements obtained from SPINOE ranged from a factor of 40 to 70 , with variations depending on the starting xenon polarization and $\mathrm{Xe} / \mathrm{CS}_{2}$ molar ratio (range 1-5). Enhancements obtainable from thermal mixing were always bigger than those from SPINOE, with an observed maximum at a field $B_{\text {mix }}=5 \mathrm{mT}$, where the thermal mixing is 4.7 times more efficient than SPINOE. 


\section{Low-field MRI}

Performing NMR and imaging at very low fields (even below Earth's field of $0.05 \mathrm{mT}$ ) with only a small number of averages is currently not possible using thermally polarized protons. In this respect, too, the use of hyperpolarization opens an unprecedented range of applications.

The general trend in proton MRI has been towards higher magnetic fields. At present, the typical field for clinical MR scanners is shifting from $1.5 \mathrm{~T}$ to $3 \mathrm{~T}$. Only a handful higher field whole body scanners (4 T to $9.4 \mathrm{~T}$ ) are available worldwide.

Hyperpolarized gases already have a high (non-equilibrium) magnetization, such that the increase of Boltzmann polarization due to high magnetic field is at best uninteresting (the benefit of zero background, coming from the non-renewable nature of magnetization, is increasingly spoiled at very high fields). The requirement of resolving lines with different chemical shifts (e.g. Xe in blood and tissues) implies moderate to high field. The same is valid for resolving exchange between different environments. The longer $T_{1}$ at higher fields is beneficial for the survival of polarization through the delivery process.

However, since the magnitude of SPINOE depends on the equilibrium polarization of the species on which polarization is to be transferred, low fields are ideal for the study of this effect. In cases where $J$-couplings are of interest, a low field is equally well suited as a high one. From the discussion of sections 6.1 and 6.2, the benefits of low field are clear, from the point of view of SNR and minimization of the effects of susceptibility heterogeneity. The reduction of the effects of susceptibility gradients (which scale with the applied field) is an important aspect in lung imaging; the gas-filled lung alveoli of the size of $0.1 \mathrm{~mm}$ give rise to magnetic field gradients of $0.1 \mathrm{~T} \mathrm{~m}^{-1}$ in an applied field of $1 \mathrm{~T}$. Gas diffusion taking place in the presence of such high local field gradients is a source of severe signal loss (Darrasse et al 1997, Leduc et al 1998). In an effort to minimize the effects of susceptibility heterogeneity, very low field $\left(<15 \mathrm{mT}\right.$ ) lung imagers using hyperpolarized ${ }^{3} \mathrm{He}$ as well as ${ }^{129} \mathrm{Xe}$ have been developed (Bidinosti et al 2003, Wong et al 1999, Venkatesh et al 2003, Mair et al 2004).

At sufficiently low-field, MRI may even become available to patients with metallic prostheses or implanted electronic equipment as pacemakers, who are largely denied the benefits of this imaging modality at clinical fields. An important factor to be considered is the much lower cost of low-field magnets compared to high-field superconducting ones (a factor unfortunately somewhat diminished by the high cost of a commercial polarizer).

Low-field studies can be classified by the detection method, since this provides the most stringent limit to how low a low-field can still be used: either conventional or SQUID-based detection. Conventional low-field applications are discussed in an excellent review by Mair and Walsworth (2002).

The diagnostic methods for characterization of the degree of polarization achieved by optical pumping followed by spin exchange make use of NMR of HypXe or He at fields of typically a few hundred Gauss. In this simple form, low-field NMR of polarized gases with conventional detection methods has been employed from the very beginning of their production.

Solenoid-detected 1D images of ${ }^{3} \mathrm{He}$ at a field of $31 \mathrm{G}$ were reported quite early-on Saam et al (1996). The first demonstration of very low-field xenon NMR followed shortly afterwards. TonThat et al studied the relaxation of polarized Xe, extending the information on $T_{1}$ times and relaxation mechanism down to $0.019 \mathrm{mT}$ (TonThat et al 1997). A spectrometer based on a dc SQUID detector and superconducting pickup coil (TonThat and Clarke 1996) was used to detect signals at these extremely low frequencies (down to $200 \mathrm{~Hz}$ ). As a result, the decrease of $T_{1}$ with magnetic field down to $\sim 2 \mathrm{mT}$ was determined, and a relatively constant value of $T_{1}$ of $2000 \mathrm{~s}$ was measured below $2 \mathrm{mT}$ and persisting at least down to $0.02 \mathrm{mT}$. 
Using the same SQUID-based spectrometer, Augustine et al (1998) report magnetic resonance imaging with both hyperpolarized ${ }^{3} \mathrm{He}$ gas at a field of $0.54 \mathrm{mT}$ and solid hyperpolarized xenon at $4.2 \mathrm{~K}$ and $1 \mathrm{mT}$. The sample, field-producing and gradient coil are immersed in liquid helium. Only one gradient, of strength $46 \mathrm{mT} \mathrm{m}^{-1}$ is used to encode the spatial information (a profile). For the 2D reconstruction, the object is rotated around its axis in 12 angular steps, and the corresponding profiles are acquired. A resolution of $1 \mathrm{~mm}$ is reported for the reconstructed 2D image of xenon frozen in a glass cylinder.

Low-field 2D imaging with conventional detection and using a spectrometer with roomtemperature components was first demonstrated by Tseng et al (1998) using the strong signal from polarized ${ }^{3} \mathrm{He}$, in a field of only $21 \mathrm{G}(2.1 \mathrm{mT})$. The dramatic reduction of the effect of susceptibility gradients compared to high fields was demonstrated, as well as the possibility to image void spaces within conductors, due to the larger penetration of low frequency RF. For selected applications, the images of spaces filled with polarized He at $2.1 \mathrm{mT}$ are of better quality than those of the same spaces filled with water and imaged at $4.7 \mathrm{~T}$. In imaging with polarized $\mathrm{He}$ gas, diffusion and not SNR was found to play the limiting role in determining the resolution (around $1 \mathrm{~mm}$ for unrestricted diffusion). The method was further developed by the same group and a complete, low cost system for low-field imaging of hyperpolarized gases has been reported by Wong et al (1999). Several aspects relevant to both low field (SNR, susceptibility heterogeneity, RF penetration depth) and imaging with hyperpolarized gases (radiation damping) have been discussed in depth by Wong et al (1999). Examples of ${ }^{3} \mathrm{He}$ imaging at $20.6 \mathrm{G}$ were presented, including the image of an excised rat lung with $T_{2}^{*}$ values longer than $100 \mathrm{~ms}$ (compared to $5 \mathrm{~ms}$ measured at high field). This is due to the low-field suppression of the effects of severe susceptibility heterogeneity present in the lungs.

Very low-field $(15 \mathrm{mT})$ hyperpolarized gas MRI of the lungs in vivo was demonstrated by Venkatesh et al $(2001,2003)$. Rat lung images at $15 \mathrm{mT}$ were obtained with both ${ }^{3} \mathrm{He}$ and ${ }^{129} \mathrm{Xe}$, and images of the human lungs with ${ }^{3} \mathrm{He}$. The SNR was satisfactory, despite the rather low polarization degree of only $5 \%$. There is a clear advantage in using ${ }^{3} \mathrm{He}$ instead of xenon for this application, due to the higher $\gamma$ of helium.

The feasibility of in vivo imaging at low field with hyperpolarized xenon and conventional encoding and detection methods was further discussed in a recent study by Cross et al (2003). It was suggested that a field of at least $\sim 50 \mathrm{mT}$ is necessary for imaging of small animals with reasonable SNR, and that the largest increase in SNR is expected to come from increase in the polarization degree (a factor of around 3 only is expected to come from improving the hardware).

The sensitivity of xenon to its environment was discussed in detail in the previous sections. One of the major disadvantages in performing low-field xenon NMR/MRI, contrasted to highfield experiments, is the loss of chemical shift information. Saxena et al (2001) report a proof of principle that SQUID NMR can recover part of this valuable information. The xenon gas peak was separated from that of xenon adsorbed on polypropylene in a field of $2.54 \mathrm{mT}$. The $200 \mathrm{ppm}$ difference corresponds to $\sim 6 \mathrm{~Hz}$, which can be readily resolved. The main source of line broadening is the inhomogeneity of the applied field, which can be corrected by shim coils, and not the susceptibility heterogeneity which is inherent to the sample. An SNR of 20 was obtained after 100 averages. Because of the high sensitivity of the SQUID detector, a water signal could also be obtained, after 40000 averages. The same group (Schlenga et al 1999) previously demonstrated 2D imaging with SQUID detection of the 30000 -averaged signal from thermally polarized oil protons at $2 \mathrm{mT}$. Further improvements in SQUID-detected NMR have been proposed. McDermott et al (2002) have demonstrated detection of $5 \mathrm{ml}$ mineral oil by prepolarizing the sample in a field of $1.8 \mathrm{mT}$ and detecting the magnetic flux at $1.8 \mu \mathrm{T}$ (lower noise, much narrower lines, higher SNR). Not only can the method resolve different 
nuclear species (protons and ${ }^{31} \mathrm{P}$ ), but also a doublet in the proton line, due to electron mediated $J$-coupling between ${ }^{1} \mathrm{H}$ and ${ }^{31} \mathrm{P}$, could be detected and the $J$-coupling constant measured. The technique of bandwidth narrowing due to detection in extremely low fields, where the linewidth approaches the intrinsic (lifetime) limit, is suggested to be applicable to low-field MRI as well. The work on low-field MRI with chemical shift resolution of hyperpolarized xenon is further refined by the Berkeley group (Wong-Foy et al 2002). Using SQUID detection, 2D images of hyperpolarized xenon flowing through and adsorbed by crushed aerogel were obtained in a field of $2.3 \mathrm{mT}$. An SNR of 30 was obtained from $1 \mathrm{ml}$ xenon gas at $1 \mathrm{~atm}$ after 100 scans, compared to SNR of 3 for the same volume of mineral oil at the same frequency $(27 \mathrm{kHz}$, $0.62 \mathrm{mT}$ for proton), after 80000 averages. An image resolution of $1 \mathrm{~mm}$ was obtained with gradients of only $1 \mathrm{mT} \mathrm{m}^{-1}$. The $200 \mathrm{ppm}$ chemical shift difference between free gas and adsorbed xenon can be well resolved for a flow rate of $0.59 \mathrm{ml} \mathrm{s}^{-1}$, and barely so for $1.57 \mathrm{ml} \mathrm{s}^{-1}$. The line broadening arises from field inhomogeneity and gas flow with exchange between free and adsorbed xenon. Improvements are well possible; it is anticipated that by using a stop-flow apparatus and a homogeneous magnet with shim coils, chemical shifts of the order of $10 \mathrm{ppm}$ could be resolved, making chemical shift imaging possible at ultra low fields.

A different and quite spectacular way of improving both NMR and MRI at very low field, called remote detection, was recently proposed and exemplified by the Berkeley group (Moule et al 2003). The idea is to separate the encoding and detection steps in space as well as in time, allowing optimization of each step separately. A simple example of encoding is by using a $\pi / 2$ pulse followed by an evolution time, followed by a second $\pi / 2$ pulse, resulting in information about the evolution time stored as longitudinal magnetization. The magnetization is then physically transported to the detection station. The magnetization inverted in a low field of 4-7 mT is read out in a field of $4.3 \mathrm{~T}$ after travelling for about $8 \mathrm{~s}$ and $5 \mathrm{~m}$. Information about chemical shift (xenon in aerogel) at the encoding place can be transferred to the detection station although only gaseous xenon is directly detected. Furthermore, a 2D image encoded at low field can be detected in high field. All the advantages of low-field encoding (for example minimizing susceptibility artefacts) are kept, and conventional detection at high field can be used. Moule et al estimate that by detecting in a field 1000 times stronger than that used for encoding, amplification of the SNR by a factor of 180 is realized. An application to remotely detected MRI of porous samples has been recently reported (Seeley et al 2004).

Another exciting direction for the use of hyperpolarized gases in extremely low fields is sketched by the recent results of Heckman et al (2003). They obtain an enhancement factor of $10^{6}$ for a proton signal in a field of $1 \mu \mathrm{T}$, due to its contact with and cross-relaxation from hyperpolarized xenon. The low frequency of $40 \mathrm{~Hz}$ for protons and $11.2 \mathrm{~Hz}$ for xenon is detected by two high temperature SQUID magnetometers. The huge enhancement of the proton signal is due to the same SPINOE effect reported to produce an enhancement of 1000 in the proton signal in a field of $1 \mathrm{mT}$ (Appelt et al 2001). An impressive study of the effects of the heteronuclear long-range dipolar effects is performed as well, and highlights the dependence of the proton as well as xenon transversal relaxation time $\left(T_{2}^{*}\right)$ on the xenon magnetization.

The record for lowest field relaxation time measurement to date is probably held by the group of Physikalisch-Technische Bundesanstalt Berlin (Kilian 2001, Rinneberg et al 2000), who measured $T_{2}$ values of up to $8000 \mathrm{~s}$ from hyperpolarized Xe gas under pressure of 40-900 mbar at a field as low as $4.5 \mathrm{nT}$ (Larmor frequency 1/18 Hz!). A single channel dc SQUID gradiometer inside a magnetically shielded room was used for detection of the free precession of xenon magnetization. The spatial distribution of magnetization was analysed using an MEG (magnetoencephalography) system for medical applications composed of 37 SQUID detectors. 
A glimpse of the exciting future of optical-pumping-based applications is provided by the recent results of the Princeton group (Allred et al 2002, Kominis et al 2003). A subfemtotesla multichannel atomic magnetometer is reported by Kominis, Kornack, Allred and Romalis, with a theoretical sensitivity limit below $0.01 \mathrm{fT} \mathrm{Hz}^{-1 / 2}$. Localization of magnetic field sources with a resolution of $2 \mathrm{~mm}$ is demonstrated with the device, opening the way to, among others, unprecedented insight into the realm of biomagnetism.

\section{Conclusions}

NMR and MRI are intrinsically low SNR methods when compared to other forms of spectroscopy. This inherent problem has ensured that any possibility of increasing the SNR is greeted with enthusiasm. The use of hyperpolarized gases offers the opportunity to increase the SNR by orders of magnitude; in comparison, increasing the SNR by increasing the static magnetic field strength pales into insignificance. As discussed above, the advantageous use of hyperpolarized xenon has been amply demonstrated in materials research and at low fields. For in vivo applications, however, relaxation times are a very important consideration in determining experimental feasibility. Much of the research, for both Xe and ${ }^{3} \mathrm{He}$, has focused on the lung since delivery of the gas by inhalation is much less problematic. The early promise of in vivo application in the brain and other distal organs has been largely unrealized because of the enhanced relaxation rates in vivo. Development of carrier technology offers a potential route to future progress. The many extremely desirable characteristics of xenon could then be fully exploited to fulfil the visions of the early investigators. This remains a tantalizing prospect!

\section{References}

Albert M S and Balamore D 1998 Development of hyperpolarized noble gas MRI Nucl. Instrum. Methods Phys. Res. 402 441-53

Albert M S, Balamore D, Kacher D F, Venkatesh A K and Jolesz F A 2000 NMR Biomed. 13 407-14

Albert M S, Balamore D, Sakai K, Kacher D, Walsworth R L, Oteiza E and Jolesz F A 1996 Proc. ISMRM, 4th Annual Meeting p 1357

Albert M S, Cates G D, Driehuys B, Happer W, Saam B, Springer C S Jr and Wishnia A 1994 Biological magnetic resonance imaging using laser-polarized ${ }^{129}$ Xe Nature 370 199-201

Albert M S, Kacher D F, Balamore D, Venkatesh A K and Jolesz F A $1999 T_{1}$ of ${ }^{129}$ Xe in blood and the role of oxygenation J. Magn. Reson. $140264-73$

Albert M S, Schepkin V D and Budinger T F 1995 Measurement of ${ }^{129}$ Xe T1 in blood to explore the feasibility of hyperpolarized ${ }^{129}$ Xe MRI J. Comput. Assist. Tomogr. $19975-8$

Allred J C, Lyman R N, Kornack T W and Romalis M V 2002 High-sensitivity atomic magnetometer unaffected by spin-exchange relaxation Phys. Rev. Lett. 89130801

Altes T A and De Lange E E 2003 Applications of hyperpolarized helium-3 gas magnetic resonance imaging in pediatric lung disease Top. Magn. Reson. Imaging 14231

Appelt S, Ben-Amar Baranga A, Erickson C J, Romalis M V, Young A R and Happer W 1998 Theory of spin-exchange optical pumping of ${ }^{3} \mathrm{He}$ and ${ }^{129} \mathrm{Xe}$ Phys. Rev. A 581412

Appelt S, Haesing F W, Baer-Lang S, Shah N J and Blumich B 2001 Proton magnetization enhancement of solvents with hyperpolarized xenon in very low-magnetic fields Chem. Phys. Lett. 348 263-9

Appelt S, Ünlü T, Zilles K, Shah N J, Baer-Lang S and Halling H 1999 Experimental studies of rubidium absolute polarization at high temperatures Appl. Phys. Lett. 75 427-9

Ardenkjaer-Larsen J H, Fridlund B, Gram A, Hansson G, Hansson L, Lerche M H, Servin R, Thaning M and Golman K 2003 Increase in signal-to-noise ratio of $>10,000$ times in liquid-state NMR Proc. Natl. Acad. Sci. USA 100 10158-63

Augustine M P, Wong-Foy A, Yarger J L, Tomaselli M, Pines A, TonThat D M and Clarke J 1998 Low field magnetic resonance images of polarized noble gases obtained with a dc superconducting quantum interference device Appl. Phys. Lett. 72 1908-10 
Augustine M P and Zilm K W 1996 Optical pumping magnetic resonance in high magnetic fields: characterization of nuclear relaxation during pumping J. Chem. Phys. 105 2998-3011

Augustine M P and Zilm K W 1997 Optical pumping magnetic resonance in high magnetic fields: measurement of high field spin exchange cross sections Chem. Phys. Lett. 280 24-30

Babcock E, Nelson I, Kadlecek S, Driehuys B, Anderson L W, Hersman F W and Walker T G 2003 Hybrid spinexchange optical pumping of ${ }^{3} \mathrm{He}$ Phys. Rev. Lett. 88123003

Becker J et al 1996 J. Neutron Res. 51

Becker J et al 1998 Interdisciplinary experiments with polarized ${ }^{3} \mathrm{He}$ Nucl. Instrum. Methods Phys. Res. A 402 $327-36$

Ben-Amar Baranga A, Appelt S, Romalis M V, Erickson C J, Young A R, Cates G D and Happer W 1998a Polarization of ${ }^{3} \mathrm{He}$ by spin exchange with optically pumped $\mathrm{Rb}$ and $\mathrm{K}$ vapours Phys. Rev. Lett. 802801

Ben-Amar Baranga A, Appelt S, Young A R and Happer W 1998b Alkalimetal-atom polarization imaging in high pressure optical-pumping cells Phys. Rev. A $\mathbf{5 8} 2282-6$

Bhaskar N D, Happer W and McClelland T 1982 Efficiency of spin exchange between rubidium spins and ${ }^{129}$ Xe nuclei in a gas Phys. Rev. Lett. 49 25-29

Bidinosti C P, Choukeife J, Nacher P J and Tastevin G 2003 In vivo NMR of hyperpolarized ${ }^{3} \mathrm{He}$ in the human lung at very low magnetic fields J. Magn. Reson. 162 122-32

Bifone A, Wolber J and Shah N J 1999 Magnetic Resonance and Brain Function: Approaches from Physics (Bristol: Institute of Physics Publishing)

Bifone A, Song Y Q, Seydoux R, Taylor R E, Goodson B M, Pietrass T, Budinger T F, Navon G and Pines A 1996 NMR of laser-polarized xenon in human blood Proc. Natl. Acad. Sci. USA 93 12932-6

Biškup N, Kalechofsky N and Candela D 2003 Spin polarization of xenon films at low-temperature induced by ${ }^{3} \mathrm{He}$ Physica B 329-333 437-8

Bouchiat M R, Carver T R and Varnum C M 1960 Nuclear polarization in ${ }^{3} \mathrm{He}$ gas induced by optical pumping and dipolar exchange Phys. Rev. Lett. 5 373-5

Bowers C R, Long H W, Pietrass T, Gaede H C and Pines A 1993 Cross-polarization from laser-polarized solid xenon to ${ }^{13} \mathrm{CO}_{2}$ by low-field thermal mixing Chem. Phys. Lett. 205 168-70

Brinkmann D, Brun E and Staub H H 1962 Helv. Phys. Acta 35431

Brossler J and Kastler A 1949 Compt. Rend. 2291213

Brossler J, Kastler A and Winter J 1952 J. Phys. Radium 13668

Brun E, Oeser J, Staub H H and Telschow C G 1954a The nuclear magnetic moments of ${ }^{129}$ Xe and ${ }^{131}$ Xe Phys. Rev. 93904

Brun E, Oeser J, Staub H H and Telschow C G 1954b Helv. Phys. Acta 27173

Brunner E 1999a Applications of laser-polarized ${ }^{129}$ Xe under continuous flow Magn. Reson. Chem. 37 S14-22

Brunner E 1999b Enhancement of surface and biological magnetic resonance using laser-polarized noble gases Concepts Magn. Reson. 11 313-35

Brunner E, Haake M, Pines A, Reimer J A and Seydoux R 1998a Enhancement of NMR signals in solid C60 and C70 using laser-polarized xenon Chem. Phys. Lett. 290 112-6

Brunner E, Seydoux R, Haake M, Pines A and Reimer J A 1998b Surface NMR using laser-polarized ${ }^{129}$ Xe under magic angle spinning conditions J. Magn. Reson. $130145-8$

Butler P, Mair R W, Hoffmann D, Hrovat M I, Rogers R A, Topulos G P, Walsworth R L and Patz S 2002 Measuring surface-area-to-volume ratios in soft porous materials using laser-polarized xenon interphase exchange nuclear magnetic resonance J. Phys.: Condens. Matter 14 L297-304

Calaprice F P, Happer W, Schreiber D F, Lowry M M, Miron E and Zeng X 1985 Nuclear alignment and magnetic moments of ${ }^{133} \mathrm{Xe},{ }^{133} \mathrm{Xe}^{m}$, and ${ }^{131} \mathrm{Xe}^{m}$ by spin exchange with optically pumped ${ }^{87} \mathrm{Rb}$ Phys. Rev. Lett. 54 $174-7$

Callaghan P T 1991 Principles of Nuclear Magnetic Resonance Microscopy (Oxford: Clarendon)

Callot V, Canet E, Brochot J, Berthezene Y, Viallon M, Humblot H, Briguet A, Tournier H and Cremillieux Y 2001a Vascular and perfusion imaging using encapsulated laser-polarized helium MAGMA 12 16-22

Callot V, Canet E, Brochot J, Viallon M, Humblot H, Briguet A, Tournier H and Cremillieux Y 2001b MR perfusion imaging using encapsulated laser-polarized ${ }^{3} \mathrm{He}$ Magn. Reson. Med. 46 535-40

Carver T R and Slichter C P 1956 Experimental verification of the Overhauser nuclear polarization effect Phys. Rev. $102975-80$

Cates G D, Fitzgerald R J, Barton A S, Bogorad P, Gatzke M, Newbury N R and Saam B 1992 Rb- ${ }^{129}$ Xe spin-exchange rates due to binary and three-body collisions at high Xe pressures Phys. Rev. A 45 4631-9

Chann B, Nelson I A, Anderson L W, Driehuys B and Walker T G $2002{ }^{129}$ Xe-Xe molecular spin relaxation Phys. Rev. Lett. 88113201

Chawla M S, Chen X J, Cofer G P, Hedlund L W, Kerby M B, Ottoboni T B and Johnson G A 2000 Hyperpolarized ${ }^{3} \mathrm{He}$ microspheres as a novel vascular signal source for MRI Magn. Reson. Med. 43 440-5 
Chawla M S, Chen X J, Möller H E, Cofer G P, Wheeler C T, Hedlund L W and Johnson G A 1998 In vivo magnetic resonance vascular imaging using laser-polarized ${ }^{3} \mathrm{He}$ microbubbles Proc. Natl. Acad. Sci. USA 95 10832-5

Cherubini A and Bifone A 2003 Hyperpolarised xenon in biology Prog. Nucl. Magn. Reson. Spectrosc. 42 1-30

Cherubini A, Payne G S, Leach M O and Bifone A 2003 Hyperpolarising ${ }^{13} \mathrm{C}$ for NMR studies using laser-polarised ${ }^{129}$ Xe: SPINOE vs thermal mixing Chem. Phys. Lett. 371 640-4

Chupp T and Swanson S 2001 Medical imaging with laser polarized noble gases Adv. At. Mol. Opt. Phys. 45 41-101

Clever H L (ed) 1979 Krypton, Xenon and Radon-Gas Solubilities vol 2 (Oxford: Pergamon)

Colegrove F D and Franken P A 1960 Optical pumping of helium in the ${ }^{3} \mathrm{~S}_{1}$ metastable state Phys. Rev. 119 680-90

Colegrove F D, Schaerer L D and Walters G K 1963 Polarization of ${ }^{3} \mathrm{He}$ gas by optical pumping Phys. Rev. 132 2561-72

Cross A R, McDonald M, Parra Robles J and Santyr G E 2003 Laser-polarized ${ }^{129}$ Xe NMR at 1.88 T and 8.5 mT: a signal-to-noise ratio comparison J. Magn. Reson. 162 241-9

Cullen S C, Eger E I, Cullen B F and Gregory P 1969 Observations on the anesthetic effect of the combination of xenon and halothane Anaesthesiology 31 305-9

Darrasse L, Guillot G, Nacher P J and Tastevin G 1997 Low field ${ }^{3}$ He nuclear magnetic resonance in human lungs C. R. Acad. Sci. Paris, IIb 324 691-700

Desvaux H, Gautier T, Le Goff G, Petro M and Berthault P 2000 Direct evidence of a magnetization transfer between laser-polarized xenon and protons of a cage-molecule in water Eur. Phys. J. D 12 289-96

Desvaux H, Huber J G, Brotin T, Dutasta J P and Berthault P 2003 Magnetization transfer from laser-polarized xenon to protons with spin-diffusion quenching Chemphyschem 4 384-7

Deville G, Bernier M and Delrieux J M 1979 NMR multiple echoes observed in solid ${ }^{3}$ He Phys. Rev. B 19 $5666-88$

Diehl P and Jokisaari J 1990a J. Magn. Reson. 88660

Diehl P and Jokisaari J 1990b ${ }^{131}$ Xe NMR in probing electric field gradients in thermotropic nematogens Chem. Phys. Lett. 165 389-91

Dimitrov I E, Reddy R and Leigh J S 2000 Intermolecular dipole-dipole relaxation of ${ }^{129}$ Xe dissolved in water J. Magn. Reson. 145 302-6

Driehuys B, Cates G D and Happer W 1995 Surface relaxation mechanisms of laser-polarized ${ }^{129}$ Xe Phys. Rev. Lett. 74 4943-6

Driehuys B, Cates G D, Happer W, Mabuchi H, Saam B, Albert M S and Wishnia A 1993 Spin transfer between laser-polarized ${ }^{129}$ Xe nuclei and surface protons Phys. Lett. A $18488-92$

Driehuys B, Cates G D, Miron E, Sauer K, Walter D K and Happer W 1996 High-volume production of laser-polarized ${ }^{129}$ Xe Appl. Phys. Lett. $691668-70$

Duhamel G, Choquet P, Grillon E, Lamalle L, Leviel J L, Ziegler A and Constantinesco A 2001 Xenon-129 MR imaging and spectroscopy of rat brain using arterial delivery of hyperpolarized xenon in a lipid emulsion Magn. Reson. Med. 46 208-12

Duhamel G et al 2000 In vivo ${ }^{129} \mathrm{Xe}$ NMR in rat brain during intra-arterial injection of hyperpolarized ${ }^{129}$ Xe dissolved in a lipid emulsion C. R. Acad. Sci. III 323 529-36

Eaton W A, Henry E R, Hofrichter J and Mozzarelli A 1999 Is cooperative oxygen binding by haemoglobin really understood? Nat. Struct. Biol. 6 351-8

Eberle B, Markstaller K, Schreiber W G and Kauczor H U 2001 Hyperpolarised gases in magnetic resonance: a new tool for functional imaging of the lung Swiss Med. Wkly. 131 503-9

Fermi E and Rasetti F 1925a Effect of an alternating magnetic field on the polarization of the resonance radiation of mercury vapour Nature 115764

Fermi E and Rasetti F 1925b Z. Phys. 33246

Fitzgerald R J, Sauer K L and Happer W 1998 Cross-relaxation in laser-polarized liquid xenon Chem. Phys. Lett. 284 87-92

Fitzsimmons W A, Tankersley L L and Walters G K 1969 Nature of surface-induced nuclear-spin relaxation Phys. Rev. 179 156-65

Fraissard J and Ito T 1988 Zeolites 8350

Franks N P and Lieb W R 1978 Where do general anaesthetics act? Nature 274 339-42

Franks N P and Lieb W R 1987 Trends Pharmacol. Sci. 8 169-74

Franks N P and Lieb W R 1994 Molecular and cellular mechanisms of general anaesthesia Nature 367 607-14

Frossati G 1998a Polarization of ${ }^{3} \mathrm{He}, \mathrm{D}_{2}$ and (eventually) ${ }^{129} \mathrm{Xe}$ using low temperatures and high magnetic fields J. Low Temp. Phys. 111521

Frossati G 1998b Polarization of ${ }^{3} \mathrm{He}, \mathrm{D}_{2}$ (and possibly ${ }^{129} \mathrm{Xe}$ ) using cryogenic techniques Nucl. Instrum. Methods Phys. Res. A 402 479-83 
Fukutomi J, Suzuki E, Shimizu T, Kimura A and Fujiwara H 2003 Analysis of the effect of foreign gases in the production of hyperpolarized ${ }^{129} \mathrm{Xe}$ gas on a simple system working under atmospheric pressure J. Magn. Reson. $16026-32$

Fung Y C, Tsang W C and Patitucci P 1981 High-resolution data on the geometry of red blood cells Biorheology 18 369-85

Gamblin R L and Carver T R 1965 Polarization and relaxation processes in ${ }^{3} \mathrm{He}$ gas Phys. Rev. 138 A946-60

Gast K K, Eberle B, Schmiedeskamp J and Kauczor H U 2003 Magnetic resonance imaging using hyperpolarized He-3-gas Acad. Radiol. $101119-31$

Gatzke M, Cates G D, Driehuys B, Fox D, Happer W and Saam B 1993 Extraordinarily slow nuclear spin relaxation in frozen laser-polarized ${ }^{129}$ Xe Phys. Rev. Lett. 70 690-3

Golman K, Ardenkjaer-Larsen J H, Petersson J S, Mansson S and Leunbach I 2003 Molecular imaging with endogenous substances Proc. Natl. Acad. Sci. USA 100 10435-9

Goodson B M 1999 Using injectable carriers of laser-polarized noble gases for enhancing NMR and MRI Concepts Magn. Reson. $11203-23$

Goodson B M 2002 Nuclear magnetic resonance of laser-polarized noble gases in molecules, materials, and organisms J. Magn. Reson. 155 157-216

Goodson B M, Song Y, Taylor R E, Schepkin V D, Brennan K M, Chingas G C, Budinger T F, Navon G and Pines A 1997 In vivo NMR and MRI using injection delivery of laser-polarized xenon Proc. Natl. Acad. Sci. USA 94 14725-9

Grover B C 1978 Noble-gas NMR detection through noble-gas-rubidium hyperfine contact interaction Phys. Rev. Lett. 40 391-2

Haake M, Goodson B M, Laws D D, Brunner E, Cyrier M C, Havlin R H and Pines A 1998 NMR of supercritical laser-polarized xenon Chem. Phys. Lett. 292 686-90

Han S, Kühn H, Häsing F W, Münnemann K, Blümich B and Appelt S 2004 Time resolved spectroscopic NMR imaging using hyperpolarized ${ }^{129}$ Xe J. Magn. Reson. $167298-305$

Hanle W 1924 Z. Phys. 3093

Happer W 1972 Optical pumping Rev. Mod. Phys. 44 169-249

Haselmeier R, Holz M, Marbach W and Weingartner H 1995 Water dynamics near a dissolved noble gas. First direct experimental evidence for a retardation effect J. Phys. Chem. $992243-6$

Heckman J J, Ledbetter M P and Romalis M V 2003 Enhancement of SQUID-detected NMR signals with hyperpolarized liquid ${ }^{129} \mathrm{Xe}$ in a 1 microT magnetic field Phys. Rev. Lett. 91067601

Hedlund L W and Johnson G A 2002 Mechanical ventilation for imaging the small animal lung ILAR J. 3 159

Hedlund L W, Möller H E, Chen X J, Chawla M S, Cofer G P and Johnson G A 2000 Mixing oxygen with hyperpolarized ${ }^{3} \mathrm{He}$ for small-animal lung studies NMR Biomed. 13 202-6

Hoult D I and Richards R E 1976 The signal-to-noise ratio of the nuclear magnetic resonance experiment J. Magn. Reson. 2471

Hunt E R and Carr H Y 1963 Nuclear magnetic resonance of ${ }^{129}$ Xe in natural xenon Phys. Rev. $1302302-5$

Ito T and Fraissard J 1980 5th Int. Conf. on Zeolites (Heyden, Naples) ed L V C Rees p 510

Jameson A K, Jameson C J and Gutowsky H S 1970 Density dependence of ${ }^{129}$ Xe chemical shifts in mixtures of xenon and other gases J. Chem. Phys. 532310

Jameson C J, Jameson A K and Cohen S M 1973 Temperature and density dependence of ${ }^{129}$ Xe chemical shift in xenon gas J. Chem. Phys. 59 4540-6

Jameson C J, Jameson A K and Hwang J K 1988 Nuclear-spin relaxation by intermolecular magnetic dipole coupling in the gas phase- ${ }^{129} \mathrm{Xe}$ in oxygen J. Chem. Phys. 89 4074-81

Jänsch H J, Hof T, Ruth U, Schmidt J, Stahl D and Fick D 1998 NMR of surfaces: sub-monolayer sensitivity with hyperpolarized ${ }^{129}$ Xe Chem. Phys. Lett. $296146-50$

Jau Y-Y, Kuzma N N and Happer W 2002 High-field measurement of the ${ }^{129} \mathrm{Xe}-\mathrm{Rb}$ spin-exchange rate due to binary collisions Phys. Rev. A 66052710

Jeener J 1999 Dynamical effects of the dipolar field inhomogeneities in high-resolution NMR: spectral clustering and instabilities Phys. Rev. Lett. 82 1772-5

Jeener J 2002 Dynamical instabilities in liquid nuclear magnetic resonance experiments with large nuclear magnetization, with and without pulsed field gradients J. Chem. Phys. 116 8439-46

Jokisaari J 1994 NMR of noble gases dissolved in isotropic and anisotropic liquids Prog. Nucl. Magn. Reson. Spectrosc. 26 1-26

Julbe A et al $1999{ }^{129}$ Xe NMR investigations for the textural characterization of sol-gel derived amorphous microporous silica J. Porous Mater. 6 41-54 
Kadlecek S, Anderson L W and Walker T 1998 Measurement of potassium-potassium spin relaxation cross-sections Nucl. Instrum. Methods Phys. Res. 402 208-11

Kastler A 1950 Quelques Suggestions concernant la production optique et la detection optique d'une inegalite de population des niveaux de quantification spatiale des atomes: application a l'experience de Stern et Gerlach et a la resonance magnetique J. Phys. Paris $11255-65$

Kauczor H U, Chen X J, Van Beek E J R and Schreiber W G 2001 Pulmonary ventilation by magnetic resonance: at the doorstep of clinical application Eur. Respir. J. 17 1008-23

Kauczor H U, Surkau R and Roberts T 1998 MRI using hyperpolarized noble gases Eur. Radiol. 8 820-7

Kauczor H U, Hanke A and Van Beek E J 2002 Assessment of lung ventilation by MR imaging: current status and future perspectives Eur. Radiol. 12 1962-70

Kilian W 2001 PhD Thesis Freie Universität Berlin, unpublished

Kilian W, Seifert F and Rinneberg H 2004a Dynamic NMR spectroscopy of hyperpolarized ${ }^{129}$ Xe in human brain analysed by an uptake model Magn. Reson. Med. 51 843-7

Kilian W, Seifert F and Rinneberg H 2004b Time- and spatially-resolved in vivo human brain MR-spectroscopy using hyperpolarized ${ }^{129}$ Xe Proc. ISMRM, 11th Annual Meeting p 767

Kominis I K, Kornack T W, Allred J C and Romalis M V 2003 A subfemtotesla multichannel atomic magnetometer Nature 422 596-9

Landon C, Berthault P, Vovelle F and Desvaux H 2001 Magnetization transfer from laser-polarized xenon to protons located in the hydrophobic cavity of the wheat nonspecific lipid transfer protein Protein Sci. 10 762-70

Lang S et al 2002 Increasing the spin-lattice relaxation time of hyperpolarized xenon ice at 4.2 K Appl. Phys. Lett. 80 886-7

Lavini C, Payne G S, Leach M O and Bifone A 2000 Intravenous delivery of hyperpolarized ${ }^{129}$ Xe: a compartmental model NMR Biomed. 13 238-44

Lawrence J H, Loomis W F, Tobias C A and Turpin F H 1946 Preliminary observations on the narcotic effect of xenon with a review of values for solubilities of gases in water and oils J. Physiol. 105 197-204

Leawoods J C, Saam B T and Conradi M S 2000 Polarization transfer using hyperpolarized, supercritical xenon Chem. Phys. Lett. 327 359-63

Leawoods J C, Yablonskiy D A, Saam B T, Gierada D S and Conradi M S 2001 Hyperpolarized 3Helium gas production and MR imaging of the lung Concepts Magn. Reson. 13 277-93

Leduc M, Nacher P and Tastevin G 1998 Magnetic Resonance Imaging using polarized noble gases Laser. Phys. 8 799-802

Lin Y Y, Lisitza N, Ahn S and Warren W S 2000 Resurrection of crushed magnetization and chaotic dynamics in solution NMR spectroscopy Science 290 118-22

Long H W, Gaede H C, Shore J, Reven L, Bowers C R, Kritzenberger J, Pietraß T, Pines A, Tang P and Reimer J A 1993 High-Field cross polarization NMR from laser-polarized xenon to a polymer surface J. Am. Chem. Soc. 115 8491-2

Luhmer M, Goodson B M, Song Y Q, Laws D D, Kaiser L, Cyrier M C and Pines A 1999 Study of xenon binding in cryptophane-A using laser-induced NMR polarization enhancement J. Am. Chem. Soc. $1213502-12$

Luhmer M, Moschos A and Reisse J 1995 Intermolecular dipole-dipole spin relaxation of xenon-129 dissolved in benzene. A molecular-dynamics simulation study J. Magn. Reson. 113 164-8

MacNamara E et al 1999 Cross polarization and cross relaxation from laser-polarized xenon to surface species J. Phys. Chem. B 1031158

Mair R W, Cory D G, Peled S, Tseng C H, Patz S and Walsworth R L 1998 Pulsed-field-gradient measurements of time-dependent gas diffusion J. Magn. Reson. 135 478-86

Mair R W, Hrovat M I, Patz S, Rosen M S, Ruset I C, Topulos G P, Tsai L L, Butler J P, Hersman F W and Walsworth R L 2004 Orientation-dependent ${ }^{3} \mathrm{He}$ lung imaging in an open access, very-low-field human MRI system Magn. Reson. Med. submitted (http://arXiv.org/pdf/physics/0403090)

Mair R W, Tseng C H, Wong G P, Cory D G and Walsworth R L 2000 Magnetic resonance imaging of convection in laser-polarized xenon Phys. Rev. E 61 2741-8

Mair R W and Walsworth R L 2002 Novel MRI applications of laser-polarized noble gases Appl. Magn. Reson. 22 159-73

Mair R W, Wong G P, Hoffmann D, Hurlimann M D, Patz S, Schwartz L M and Walsworth R L 1999 Probing porous media with gas diffusion NMR Phys. Rev. Lett. 83 3324-7

Mansson S, Wolber J, Driehuys B, Wollmer P and Golman K 2003 Characterization of diffusing capacity and perfusion of the rat lung in a lipopolysaccaride disease model using hyperpolarized ${ }^{129} \mathrm{Xe}$ Magn. Reson. Med.50 1170-9

Martin C C, Williams R F, Gao J H, Nickerson L D, Xiong J and Fox P T 1997 The pharmacokinetics of hyperpolarized xenon: implications for cerebral MRI J. Magn. Reson. Imaging 7 848-54 
Marx T et al 2000 Xenon anaesthesia J. R. Soc. Med. 93513

Mazzanti M L, Sun Y, Mansour J, Shah N, Doshi S S, Jolesz F and Albert M S 2004 Functional brain imaging using hyperpolarized ${ }^{129}$ Xe Proc. ISMRM, 11th Annual Meeting p 768

McDermott R, Trabesinger H A, Muck M, Hahn E L, Pines A and Clarke J 2002 Liquid-state NMR and scalar couplings in microtesla magnetic fields Science 295 2247-9

McKim S and Hinton J F 1994 Evidence of xenon transport through the gramicidin channel: a ${ }^{129}$ Xe-NMR study Biochim. Biophys. Acta 1193 186-98

Meersmann T and Haake M 1998 Magnetic field dependent xenon-131 quadrupolar splitting in gas and liquid phase NMR Phys. Rev. Lett. 81 1211-4

Meersmann T, Smith S A and Bodenhausen G 1998 Multiple-quantum filtered xenon-131 NMR as a surface probe Phys. Rev. Lett. 80 1398-401

Melville D, Paul F and Roath S 1975a High gradient magnetic separation of red-cells from whole-blood IEEE Trans. Magn. 11 1701-4

Melville D, Paul F and Roath S 1975b Direct magnetic separation of red-cells from whole-blood Nature 255706

Meyer K H 1899 Zur Theorie der Alkoholnarkose Trans. Farad. Soc. 331062

Miller K W, Reo N V, Schoot Uiterkamp A J, Stengle D P, Stengle T R and Williamson K L 1981 Xenon NMR: chemical shifts of a general anesthetic in common solvents, proteins, and membranes Proc. Natl. Acad. Sci. USA 78 4946-9

Millot Y, Man P P, Springuel-Huet M A and Fraissard J 2001 Quantification of electric-field gradient in the supercage of Y zeolites with Xe-131 ( $\mathrm{I}=3 / 2$ ) NMR C. R. Acad. Sci. II C 4 815-8

Möller H E, Chen X J, Saam B, Hagspiel K D, Johnson G A, Altes T A, de Lange E E and Kauczor H U 2002 MRI of the lungs using hyperpolarized noble gases Magn. Reson. Med. 47 1029-51

Moudrakovski I, Breeze S R, Simard B, Ratcliffe C I, Ripmeester J A, Seideman T and Tse J S 2001a Gas-phase nuclear magnetic relaxation in Xe-129 revisited J. Chem. Phys. $1142173-81$

Moudrakovski I L, Lang S, Ratcliffe C I, Simard B, Santyr G and Ripmeester J A 2000a Chemical shift imaging with continuously flowing hyperpolarized xenon for the characterization of materials J. Magn. Reson. 144 372-7

Moudrakovski I L, Ratcliffe C I and Ripmeester J A 2001b Probing transient hydrate structures with hyperpolarized Xe-129 NMR spectroscopy: a metastable structure II hydrate of Xe Angew. Chem. Int. Ed. 403890

Moudrakovski I L, Sanchez A, Ratcliffe C I and Ripmeester J A 2000b Applications of hyperpolarized xenon to diffusion in vycor porous glass J. Phys. Chem. B 104 7306-10

Moulé A J, Spence M M, Han S I, Seeley J A, Pierce K L, Saxena S and Pines A 2003 Amplification of xenon NMR and MRI by remote detection Proc. Natl. Acad. Sci. USA $1009122-7$

Mugler J P III, Bogorad P L, Driehuys B and Brookeman J R 2004a Nuclear magnetic resonance using hyperpolarized noble gases J. Magn. Reson. Anal. at press

Mugler J P III et al 1997 MR imaging and spectroscopy using hyperpolarized ${ }^{129}$ Xe gas: preliminary human results Magn. Reson. Med. 37 809-15

Mugler J P III et al 2004b The apparent diffusion coefficient of Xe-129 in the lung: preliminary human results Proc. ISMRM, 11th Annual Meeting 37 p 769

Nacher P J, Tastevin G, Villard B, Piegay N, Marion F and Sauer K 2000 NMR instabilities in spin-polarised liquids: He-3, He-3-He-4 mixtures and Xe-129 J. Low Temp. Phys. 121 743-8

National Nuclear Data Center, Brookhaven National Laboratory, Upton, NY 11973-5000; Online nuclear data file database http://www.nndc.bnl.gov/

Navon G, Song YQ, Rõom T, Appelt S, Taylor R E and Pines A 1996 Enhancement of solution NMR and MRI with laser-polarized xenon Science 271 1848-51

Overhauser A W 1953 Polarization of nuclei in metals Phys. Rev. 91476

Overton E 1901 Studien über die Narkose, zugleich ein Beitrag zur allgemeinen Pharmakologie (Jena: Fischer Verlag) Patyal B R, Gao J H, Williams R F, Roby J, Saam B, Rockwell B A, Thomas R J, Stolarski D J and Fox P T 1997 Longitudinal relaxation and diffusion measurements using magnetic resonance signals from laser-hyperpolarized ${ }^{129}$ Xe nuclei J. Magn. Reson. $12658-65$

Pauling L and Coryell C D 1936 Proc. Natl. Acad. Sci. USA 22210

Pavlovskaya G, Blue A K, Gibbs S J, Haake M, Cros F, Malier L and Meersmann T 1999 Xenon-131 surface sensitive imaging of aerogels in liquid xenon near the critical point J. Magn. Reson. 137 258-64

Peereboom P W E, Luigjes H and Prins K O 1989 An NMR spin-echo study of self-diffusion in xenon Physica A $156260-76$

Peled S, Jolesz F A, Tseng C H, Nascimben L, Albert M S and Walsworth R L 1996 Determinants of tissue delivery for ${ }^{129} \mathrm{Xe}$ magnetic resonance in humans Magn. Reson. Med. 36 340-4

Peled S, Tseng C H, Sodickson A A, Mair R W, Walsworth R L and Cory D G 1999 Single-shot diffusion measurement in laser-polarized gas J. Magn. Reson. 140 320-4 
Perutz M F 1970 Stereochemistry of cooperative effects in haemoglobin Nature 228 726-39

Perutz M F, Bolton W, Diamond R, Muirhead H and Watson H 1964 An X-ray examination of reduced horse haemoglobin Nature $203687-90$

Perutz M F, Wilkinson A J, Paoli M and Dodson G G 1998 The stereochemical mechanism of the cooperative effects in haemoglobin revisited Ann. Rev. Biophys. Biomol. Struct. 27 1-34

Pietraß T and Gaede H C 1995 Optically polarized Xe-129 in NMR-Spectroscopy Adv. Mater 7826

Pietraß T 1999 NMR of molecules and surfaces using laser-polarized xenon Colloids Surf. A 158 51-7

Pietraß T, Gaede H C, Bifone A, Pines A and Ripmeester J A 1995 Monitoring xenon clathrate hydrate formation on ice surfaces with optically enhanced Xe-129 NMR J. Am. Chem. Soc. 117 7520-5

Pietraß T, Seydoux R and Pines A 1998 Surface selective ${ }^{1} \mathrm{H} /{ }^{29} \mathrm{Si}$ CP NMR by NOE enhancement from laser polarized xenon J. Magn. Reson. 133 299-303

Plyavin Y and Blum E Y 1983 Magnetic parameters of blood cells and high-gradient paramagnetic and diamagnetic phoresis Magnetohydrodynamics 19349

Pohorille A 1998 Transient cavities in liquids and the nature of the hydrophobic effect Polish J. Chem. 72 $1680-90$

Proctor W G and Yu F C 1950 On the magnetic moments of Xe-129, Bi-209, Sc-45, Sb-121 and Sb-123 Phys. Rev. 78471

Rabi I I, Zacharias J R, Millman S and Kusch P 1938 A new method of measuring nuclear magnetic moment Phys. Rev. $\mathbf{5 3} 318$

Raftery D, Long H, Reven L, Tang P and Pines A 1992 NMR of optically pumped xenon thin-films Chem. Phys. Lett. $191385-90$

Rentzepis P M and Douglass D C 1981 Xenon as a solvent Nature 293 165-6

Rinneberg H, Kilian W, Haller A, Grosenich D, Wübbeler D, Seifert F and Trahms L 2000 Hyperpolarized ${ }^{129}$ Xe gas: SQUID detection of nuclear spin precession in low magnetic fields BIOMAG 2000: Proc. 12th Int. Conf. on Biomagnetism (Helsinki, Finland) p 222

Ripmeester J A and Ratcliffe C I 1990 On the application of ${ }^{129}$ Xe NMR to the study of microporous solids J. Phys. Chem. 94 7652-6

Romalis M V et al 1998 Toward precision polarimetry of dense polarized ${ }^{3} \mathrm{He}$ targets Nucl. Instrum. Methods Phys. Res. A 402 260-7

Romalis M V and Ledbetter M P 2001 Transverse spin relaxation in liquid ${ }^{129} \mathrm{Xe}$ in the presence of large dipolar fields Phys. Rev. Lett. 87067601

Rõõm T, Appelt S, Seydoux R, Hahn E L and Pines A 1997 Enhancement of surface NMR by laser-polarized noble gases Phys. Rev. B $5511604-10$

Rosen M S, Chupp T E, Coulter K P, Welsh R C and Swanson S D 1999 Polarized Xe-129 optical pumping/spin exchange and delivery system for magnetic resonance spectroscopy and imaging studies Rev. Sci. Instrum. 70 $1546-52$

Ruppert K, Mata J F, Brookeman J R, Hagspiel K D and Mugler J P III 2004 Exploring lung function with hyperpolarized ${ }^{129}$ Xe nuclear magnetic resonance Magn. Reson. Med. 51 676-87

Ruth U, Hof T, Schmidt J, Fick D and Jansch H J 1999 Production of nitrogen-free, hyperpolarized Xe-129 gas Appl. Phys. B 68 93-7

Saba G, Casu M and Lai A 1996 Application of quadrupolar ${ }^{131}$ Xe-NMR relaxation to the study of macromolecular systems Int. J. Quantum Chem. 59 343-8

Salerno M, Altes T A, Mugler J P III, Nakatsu M, Hatabu H and de Lange E E 2001 Hyperpolarized noble gas MR imaging of the lung: potential clinical applications Eur. J. Radiol. 40 33-44

Saam B T and Conradi M S 1998 Low frequency NMR polarimeter for hyperpolarized gases J. Magn. Reson. 134 $67-71$

Saam B, Drukker N and Happer W 1996 Edge enhancement observed with hyperpolarized He-3 Chem. Phys. Lett. 263 481-7

Sauer K L, Fitzgerald R J and Happer W 1997 Laser-polarized liquid xenon Chem. Phys. Lett. 277 153-8

Sauer K L, Fitzgerald R J and Happer W 1999 Resonance technique to probe ${ }^{129}$ Xe surface interaction Phys. Rev. A 59 R1746-9

Sauer K L, Marion F, Nacher P J and Tastevin G 2001 NMR instabilities and spectral clustering in laser-polarized liquid xenon Phys. Rev. B 63184427

Saxena S, Wong-Foy A, Moulé A J, Seeley J A, McDermott R, Clarke J and Pines A 2001 Resolution of ${ }^{129}$ Xe chemical shifts at ultralow magnetic field J. Am. Chem. Soc. 123 8133-4

Schlenga K, McDermott R, Clarke J, de Souza R E, Wong-Foy A and Pines A 1999 Low-field magnetic resonance imaging with a high-Tc dc superconducting quantum interference device Appl. Phys. Lett. 75 3695-7 
Seeley J A, Han S-I and Pines A 2004 Remotely detected high-field MRI of porous samples J. Magn. Reson. 167 282-90

Seydoux R 1995 PhD Thesis Basel, unpublished

Seydoux R, Diehl P, Mazitov R K and Jokisaari J 1993 Chemical shifts in magnetic resonance of the ${ }^{3}$ He nucleus in liquid solvents and comparison with other noble gases J. Magn. Reson. A 101 78-83

Shah N J, Appelt S, Ünlü T, Halling H and Zilles K 2001a Hochdruckpolarisator für Edelgase und Verfahren zum Betreiben des Polarisators German Patent no. DE 19937566

Shah N J, Appelt S, Ünlü T, Halling H and Zilles K 2001b High pressure polarizer for hyperpolarizing the nuclear spin of noble gases US Patent no. 6, 666047

Shah N J, Ünlü T, Wegener H P, Halling H, Zilles K and Appelt S 2000 Measurement of rubidium and xenon absolute polarization at high temperatures as a means of improved production of hyperpolarized ${ }^{129} \mathrm{Xe}$ NMR Biomed. 13 214-9

Smith T B, Chupp T E, Coulter K P and Welsh R C 1998 Recent advances in spin-exchange pumped polarized ${ }^{3} \mathrm{He}$ target technology Nucl. Instrum. Methods Phys. Res. A 402 247-9

Smith J, Knagge K, Smith L J, MacNamara E and Raftery D 2002 Investigating hyperpolarized ${ }^{129}$ Xe and CPMAS for spin polarization transfer to surface nuclei: a model study J. Magn. Res. 159 111-25

Solomon I 1955 Relaxation processes in a system of two spins Phys. Rev. 99 559-65

Song Y Q 2000 Spin polarization-induced nuclear Overhauser effect: an application of spin-polarized xenon and helium Concepts Magn. Reson. 12 6-20

Song Y Q, Goodson B M, Sheridan B, de Swiet T M and Pines A 1998a Effects of diffusion on magnetic resonance imaging of laser-polarized xenon gas J. Chem. Phys. $1086233-9$

Song Y Q, Goodson B M, Taylor R E, Laws D D, Navon G and Pines A 1997 A selective enhancement of NMR signals for alpha-cyclodextrin with laser-polarized xenon Angew. Chem. Int. Ed. Engl. 36 2368-70

Song Y Q, Taylor R E and Pines A 1998b Imaging of laser-polarized solid xenon Solid State Nucl. Magn. Reson. 10 247-50

Spees W M, Yablonskiy D A, Oswood M C and Ackerman J J 2001 Water proton MR properties of human blood at 1.5 Tesla: magnetic susceptibility, $T_{1}, T_{2}, T_{2}^{*}$, and non-Lorentzian signal behavior Magn. Reson. Med. 45 533-42

Spence M M, Rubin S M, Dimitrov I E, Ruiz E J, Wemmer D E, Pines A, Yao S Q, Tian F and Schultz P G 2001 Functionalized xenon as a biosensor Proc. Natl. Acad. Sci. USA 98 10654-7

Springuel-Huet M A, Bonardet J L, Gedeon A and Fraissard J 1999 Xe-129 NMR: overview of xenon physisorbed in porous solids Magn. Reson. Chem. 37 1-13

Stephan M, Kohlmann O, Niessen H G, Eichhorn A and Bargon J 2002 C-13 PHIP NMR spectra and polarization transfer during the homogeneous hydrogenation of alkynes with parahydrogen Magn. Reson. Chem. 40 $157-60$

Stillinger F H 1980 Water Revistied Science 209 451-7

Stith A, Hitchens T K, Hinton D P, Berr S S, Driehuys B, Brookeman J R and Bryant R G 1999 Consequences of (129)Xe-(1)H cross relaxation in aqueous solutions J. Magn. Reson. 139 225-31

Streever R L and Carr H Y 1961 Nuclear magnetic resonance of Xe-129 in natural xenon Phys. Rev. 121 20-25

Surkau R et al 1997 Realization of a broad band neutron spin filter with compressed, polarized ${ }^{3} \mathrm{He}$ gas Nucl. Instrum. Methods Phys. Res. A $\mathbf{3 8 4} 444-50$

Swanson S D, Rosen M S, Agranoff B W, Coulter K P, Welsh R C and Chupp T E 1997 Brain imaging with laser-polarized ${ }^{129}$ Xe Magn. Reson. Med. 38695

Swanson S D, Rosen M S, Coulter K P, Welsh R C and Chupp T E 1999 Distribution and dynamics of laser-polarized ${ }^{129}$ Xe magnetization in vivo Magn. Reson. Med. $421137-45$

Tilton R F Jr and Kuntz I D Jr 1982 Nuclear magnetic resonance studies of xenon-129 with myoglobin and haemoglobin Biochemistry $216850-7$

TonThat D M and Clarke J 1996 Direct current superconducting quantum interference device spectrometer for pulsed nuclear magnetic resonance and nuclear quadrupole resonance at frequencies up to $5 \mathrm{MHz}$ Rev. Sci. Instrum. 67 2890-3

TonThat D M, Ziegeweid M, Song Y Q, Munson E J, Appelt S, Pines A and Clarke J 1997 SQUID detected NMR of laser-polarized xenon at $4.2 \mathrm{~K}$ and at frequencies down to $200 \mathrm{~Hz}$ Chem. Phys. Lett. 272 245-9

Torrey H C 1963 Chemical shift and relaxation of Xe-129 in xenon gas Phys. Rev. 130 2306-12

Tseng C H, Mair R W, Wong G P, Williamson D, Cory D G and Walsworth R L 1999 Magnetic resonance imaging of laser polarized liquid xenon Phys. Rev. E 59 1785-8

Tseng C H, Peled S, Nascimben L, Oteiza E, Walsworth R L and Jolesz F A 1997 NMR of laser-polarized ${ }^{129}$ Xe in blood foam J. Magn. Reson. 126 79-86

Tseng C H, Wong G P, Pomeroy V R, Mair R W, Hinton D P, Hoffmann D, Stoner R E, Hersman F W, Cory D G and Walsworth R L 1998 Low-field MRI of laser polarized noble gas Phys. Rev. Lett. 81 3785-8 
Van Beek E J R et al 2003 Hyperpolarized 3-helium MR imaging of the lungs: testing the concept of a central production facility Eur. Radiol. $132583-6$

Venkatesh A K et al 2001 Very low-field hyperpolarized gas MRI of the lungs Proc. 'Bioastronautics Investigators' Workshop' (Galveston, TX, 17-19 Jan.)

Venkatesh A K et al 2003 MRI of the lung gas-space at very low-field using hyperpolarized noble gases Magn. Reson. Imaging 21 773-6

Venkatesh A K, Zhao L, Balamore D, Jolesz F A and Albert M S 2000 Evaluation of carrier agents for hyperpolarized xenon MRI NMR Biomed. 13 245-52

Venkatesh A K, Zhao L, Balamore D, Jolesz F A and Albert M S 2001 Hyperpolarized ${ }^{129}$ Xe MRI using gas-filled liposomes Acad Radiol. 9 S270-4

Venkatesh A K, Zhao L, Pausak T, Ward C, Jolesz F A and Albert M S 1999 Hyperpolarized gas imaging using a simple programmable gas delivery system Eur. Radiol. 9 B34

Verhulst A S, Liivak O, Sherwood M H and Chuang I L 2002 A rapid and precise probe for measurement of liquid xenon polarization J. Magn. Reson. 155 145-9

Volk C H, Kwon T M and Mark J G 1980 Measurement of the ${ }^{87}$ Rb- ${ }^{129}$ Xe spin-exchange cross-section Phys. Rev. A 21 1549-55

Walker T G and Happer W 1997 Spin-exchange optical pumping of noble-gas nuclei Rev. Mod. Phys. $69629-42$

Walter D K, Griffith W M and Happer W 2002 Magnetic slowing down of spin relaxation due to binary collisions of alkali-metal atoms with buffer-gas atoms Phys. Rev. Lett. 88093004

Weiskoff R M, Zuo C S, Boxerman J and Rosen B R 1994 Microscopic susceptibility variation and transverse relaxation-theory and experiment Magn. Reson. Med. 31 601-10

Wilson G J, Santyr G, Anderson M E and DeLuca P M Jr 1999a Proc. ISMRM, 7th Meeting p 2102

Wilson G J, Santyr G E, Anderson M E and DeLuca P M Jr 1999b Longitudinal relaxation times of ${ }^{129}$ Xe in rat tissue homogenates at 9.4 T Magn. Reson. Med. $41933-8$

Wolber J, Cherubini A, Dzik-Jurasz A S, Leach M O and Bifone A 1999a Spin-lattice relaxation of laser-polarized xenon in human blood Proc. Natl. Acad. Sci. USA $963664-9$

Wolber J, Cherubini A, Leach M O and Bifone A 2000a On the oxygenation-dependent ${ }^{129}{ }^{\mathrm{Xe}} T_{1}$ in blood $N M R$ Biomed. 13 234-7

Wolber J, Cherubini A, Leach M O and Bifone A 2000b Hyperpolarized ${ }^{129}$ Xe NMR as a probe for blood oxygenation Magn. Reson. Med. 43 491-6

Wolber J, Cherubini A, Leach M O and Bifone A 2002 Magn. Reson. Med. 47213 (erratum)

Wolber J, Cherubini A, Leach M O and Bifone A 2000c Proc. ISMRM, 8th Meeting p 969

Wolber J, Cherubini A, Santoro D, Payne G S, Leach M O and Bifone A 2000d Proc. ISMRM, 8th Meeting p 970

Wolber J, Doran S, Leach M O and Bifone A 1998 Measuring diffusion of xenon in solution with hyperpolarized ${ }^{129}$ Xe MRI Chem. Phys. Lett. $296391-6$

Wolber J, Rowland I J, Leach M O and Bifone A 1999b Perfluorocarbon emulsions as intravenous delivery media for hyperpolarized xenon Magn. Reson. Med. 41 442-9

Wong G P, Tseng C H, Pomeroy V R, Mair R W, Hinton D P, Hoffmann D, Stoner R E, Hersman F W, Cory D G and Walsworth R L 1999 A system for low field imaging of laser-polarized noble gas J. Magn. Reson. $141217-27$

Wong-Foy A, Saxena S, Moulé A J, Bitter H M, Seeley J A, McDermott R, Clarke J and Pines A 2002 Laser-polarized ${ }^{129} \mathrm{Xe}$ NMR and MRI at ultralow magnetic fields J. Magn. Reson. 157 235-41

Wu Z, Happer W and Daniels J M 1987 Coherent nuclear-spin interactions of adsorbed ${ }^{131}$ Xe gas with surfaces Phys. Rev. Lett. 59 1480-3

$\mathrm{Xu}$ Y and Tang P 1997 Amphiphilic sites for general anesthetic action? Evidence from ${ }^{129} \mathrm{Xe}-{ }^{1} \mathrm{H}$ intermolecular nuclear Overhauser effects Biochim. Biophys. Acta 1323 154-62

Yen W M and Norberg R E 1963 Nuclear magnetic resonance of ${ }^{129}$ Xe in solid and liquid xenon Phys. Rev. 131 269-75

Zeng X, Wu Z, Call T, Miron E, Schreiber D and Happer W 1985 Experimental determination of the rate constants for spin exchange between optically pumped $\mathrm{K}, \mathrm{Rb}$, and $\mathrm{Cs}$ atoms and ${ }^{129} \mathrm{Xe}$ nuclei in alkali-metal-noble-gas van der Waals molecules Phys. Rev. A 31 260-78

Zhao L et al 1996 Gradient-echo imaging considerations for hyperpolarized ${ }^{129}$ Xe MR J. Magn. Reson. 113 179-83

Zhao L, Venkatesh A K, Balamore D, Jolesz F A and Albert M S 1998 Proc. ISMRM, 6th Meeting

Zook A L, Adhyaru B B and Bowers C R 2002 High capacity production of $>65 \%$ spin polarized xenon-129 for NMR spectroscopy and imaging J. Magn. Reson. 159 175-82

Zook A L and Bowers C R 2001 High capacity production of $>40 \%$ spin polarized xenon-129 for NMR and MRI applications at the NHMFL/UF NHMFL Laboratory Spotlight http://www.magnet.fsu.edu/spotlights/ Xenon129/Xenon129po.pdf 